Supplemental Information for:

\title{
Cell-Free Biosynthesis to Evaluate Lasso Peptide Formation and Enzyme-Substrate Tolerance
}

Yuanyuan $\mathrm{Si}^{1,2}$, Ashley M. Kretsch ${ }^{1,2}$, Laura M. Daigh ${ }^{1,2}$, Mark J. Burk ${ }^{4, *}$, Douglas A. Mitchell ${ }^{1,2,3, *}$

${ }^{1}$ Carl R. Woese Institute for Genomic Biology, University of Illinois, Urbana, Illinois, United States of America; ${ }^{2}$ Department of Chemistry, ${ }^{3}$ Department of Microbiology, University of Illinois, Urbana, Illinois, United States of America; ${ }^{4}$ Lassogen, Inc., San Diego, California, United States of America

*Authors to whom correspondence should be addressed:

douglasm@illinois.edu

mburk@lassogen.com

\section{Contents}

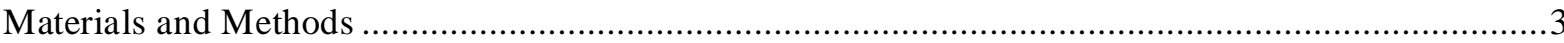

Table S1. Genetically predicted lasso peptides used for FusA chimeric precursor generation. ............11

Table S2. Nucleotide sequences of chimeric precursor peptides. ....................................................12

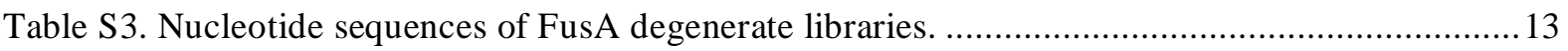

Table S4. Expected surface area for ring region among available lasso peptide structures in PDB.....14

Table S5. List of sequence-confirmed ring variants with mature lasso peptide formation...................15

Table S6. Protein accession identifiers used to mine lasso peptide BGCs from NCBI. .......................16

Table S7. Natural variability in the set of genomically predicted lasso peptides. ...............................17

Table S8. Determination of the "expected frequency" as a weighted average of the most frequent genus

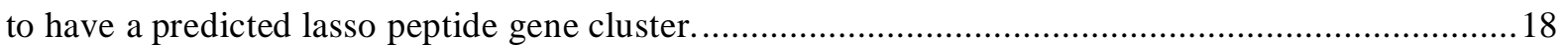

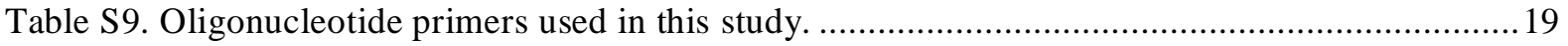

Figure S1. Plasmid maps for constructs used in CFB reactions. ...................................................22

Figure S2. High-resolution and tandem MS of burhizin produced from CFB .................................23

Figure S3. High-resolution and tandem MS of capistruin produced from CFB ................................24

Figure S4. High-resolution and tandem MS of fusilassin produced from CFB. ................................25

Figure S5. High-resolution and tandem MS of cellulassin produced from CFB ...............................26

Figure S6. Simultaneous production of multiple lasso peptides using CFB. ......................................27

Figure S7. Carboxypeptidase Y resistance of CFB-produced lasso peptides.....................................28

Figure S8. Purification and yield determination of capistruin produced from CFB ...........................29

Figure S9. MALDI-TOF-MS of Fusilassin variants with Trp1 substitutions. ......................................30

Figure S10. MALDI-TOF-MS of halolassin produced from CFB with plasmid DNA.......................31

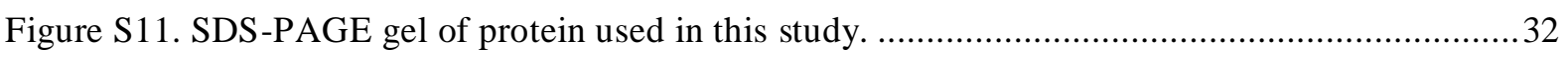

Figure S12. High-resolution and tandem MS of halolassin produced from CFB using chimeric

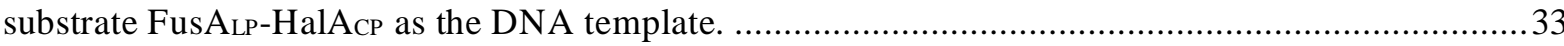

Figure S13. High-resolution and tandem MS of cellulassin produced from CFB using chimeric substrate FusA 
Figure S14. Carboxypeptidase Y treatment of halolassin and cellulassin produced with chimeric substrates from CFB.

Figure S15. Chimeric substrate processing in PURExpress reactions.

Figure S16. Replacement of the ring/loop sequences of chimeric substrates with FusA ring/loop sequence.

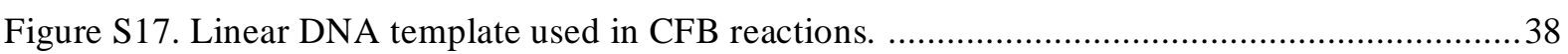

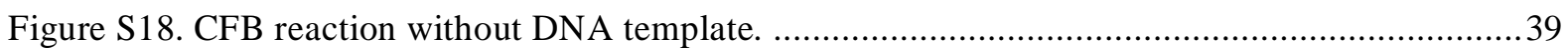

Figure S19. MALDI-TOF mass spectra of the fusilassin ring variants produced from CFB . .............40

Figure S20. High-resolution and tandem MS of TVQGA variant produced from CFB......................45

Figure S21. High-resolution and tandem MS of FASDS variant produced from CFB. .......................46

Figure S22. High-resolution and tandem MS of FTGSK variant produced from CFB.......................47

Figure S23. High-resolution and tandem MS of LSSLM variant produced from CFB......................48

Figure S24. High-resolution and tandem MS of VTTTY variant produced from CFB. .....................49

Figure S25. High-resolution and tandem MS of FTNLT variant produced from CFB. ........................50

Figure S26. High-resolution and tandem MS of NSSLW variant produced from CFB. ......................51

Figure S27. High-resolution and tandem MS of FAYMT variant produced from CFB .....................52

Figure S28. High-resolution and tandem MS of FNHLF variant produced from CFB.......................53

Figure S29. High-resolution and tandem MS of YSYFQ variant produced from CFB. ......................54

Figure S30. Carboxypeptidase Y digestion for fusilassin ring variants. .............................................56

Figure S31. Sequence logo of sequence-confirmed FusC substrates. ...............................................57

Figure S32. Simultaneous production of multiple fusilassin variants using CFB . ............................58

Figure S33. MALDI-TOF-MS spectra of the uncyclized linear core peptides of the FusC nonsubstrates

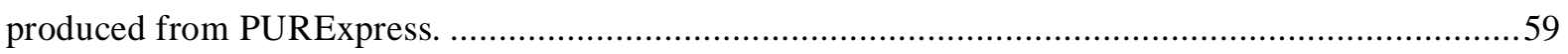

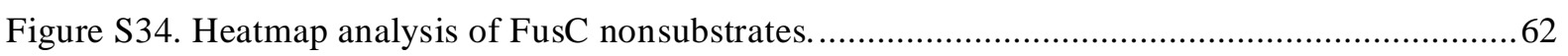

Figure S35. Position-specific amino acid analysis for lasso peptides.............................................63

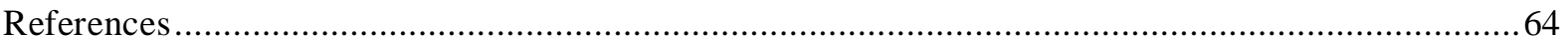




\section{Materials and Methods}

General materials and methods. The synthetic DNA encoding FusA chimeric precursor peptides were codon optimized for E. coli expression and were purchased from Twist Bioscience (Table S2). The synthetic DNA containing NNK degenerate codons (Table S3) and primers used in this study (Table S9) were purchased from Integrated DNA Technologies. Q5 DNA polymerase, restriction enzymes, T4 DNA ligase, and high efficiency Escherichia coli (E. coli) DH5a competent cells were obtained from New England Biolabs. Amicon Ultra-4 centrifugal filters were purchased from EMD Millipore. E. coli DH5a and E. coli BL21(DE3) cells were used for plasmid maintenance and protein production, respectively. Chemical reagents were from Sigma Aldrich and used without further purification unless otherwise noted. Sanger sequencing was performed by the Core DNA Sequencing Facility at the University of Illinois at Urbana-Champaign. Matrix-assisted laser desorption/ionization time-of-flight mass spectrometry (MALDI-TOF-MS) data were acquired in reflector positive mode on a Bruker UltrafleXtreme instrument (Bruker Daltonics) at the University of Illinois School of Chemical Sciences Mass Spectrometry Laboratory. MALDI-TOF-MS data analysis was carried out using the Bruker FlexAnalysis software.

Molecular biology techniques. The chimeric precursor peptides with different core sequences fused to the FusA leader region were cloned into a modified pET28 vector that provides an N-terminal maltose-binding protein (MBP) tag using 5' BamHI and 3' HindIII restriction sites. Predicted FusC substrates (core positions 2-6 varied with: MNMYM, TWTEM, NMQIY, MYTFQ, and FYNWK), predicted FusC non-substrates (IKEVT, QDWFM, FLRCL, IDRSY, and LKNFT), hybrid precursor peptides (i.e. FusA $A_{L P}-M o b A_{C P}: L I F V F P$ and FusA $\left.A_{L P}-N b s A_{C P}: L I F V F P\right)$ were prepared through PCR overlap extension. ${ }^{1}$ MBP-tagged fusilassin leader peptidase (WP_011291590.1, FusB), lasso cyclase (WP_104612995.1, FusC), and RiPP leader peptide recognition element (RRE) (WP_011291591.1, FusE) were cloned previously into pET28-MBP. ${ }^{2}$ The obtained constructs were used for heterologous expression, and pET28-MBP-FusA was used as the template for mutagenesis. Mutagenesis was performed using the QuikChange method with Q5 Polymerase. mCherry was amplified from pmCherry (Clontech) and cloned into pET28 (no MBP tag) using 5' BamHI and 3' HindIII restriction sites. The obtained construct was used to determine transcription inhibition by capistruin. All mutants were verified by Sanger sequencing.

E. coli cell lysate preparation. A single BL21(DE3) colony was inoculated into $10 \mathrm{~mL}$ of YTPG medium $\left(10 \mathrm{~g} / \mathrm{L}\right.$ yeast extract, $16 \mathrm{~g} / \mathrm{L}$ tryptone, $3 \mathrm{~g} / \mathrm{L} \mathrm{KH}_{2} \mathrm{PO}_{4}, 7 \mathrm{~g} / \mathrm{L} \mathrm{K} \mathrm{K}_{2} \mathrm{HPO}_{4}, 5 \mathrm{~g} / \mathrm{L} \mathrm{NaCl}, 18 \mathrm{~g} / \mathrm{L}$ glucose) and grown at $37{ }^{\circ} \mathrm{C}$ overnight with shaking. The culture was then used to inoculate $1 \mathrm{~L}$ of YTPG medium and grown at $37{ }^{\circ} \mathrm{C}$ with shaking. When an optical density (OD 600 ) of $\sim 0.5$ was reached, $1 \mathrm{mM}$ (final) IPTG was added. When an $\mathrm{OD}_{600}$ of $\sim 3$ was observed, the cells were harvested by centrifugation at $4,000 \times g$ for $20 \mathrm{~min}$ at $4{ }^{\circ} \mathrm{C}$. The cells were washed twice with $2 \times 400 \mathrm{~mL}$ of cold buffer S30A [10 mM Tris, $\mathrm{pH}=8.2,14 \mathrm{mM}$ magnesium acetate, $60 \mathrm{mM}$ potassium glutamate, 2 $\mathrm{mM}$ 1,4-dithiothreitol (DTT)]. After the second wash, the cell pellet was resuspended in $\sim 40 \mathrm{~mL}$ of cold buffer S30A in a pre-weighed $50 \mathrm{~mL}$ conical tube, followed by centrifugation at $5,000 \times g$ for 10 $\min$ at $4{ }^{\circ} \mathrm{C}$. The cell pellet was then weighted and resuspended in buffer S30A at $1 \mathrm{~mL} / \mathrm{g}$ cell. The cells were lysed with a homogenizer (single pass, 20,000-30,0000 psi), then subjected to 
centrifugation at $30,000 \times \mathrm{g}$ for $30 \mathrm{~min}$ at $4{ }^{\circ} \mathrm{C}$. A Pierce Bradford assay was used to determine the total protein concentration in the cell extract $(\sim 38 \mathrm{mg} / \mathrm{mL})$. The cell extract was aliquoted and stored at $-80{ }^{\circ} \mathrm{C}$ until needed.

Energy buffer preparation. The energy buffer was prepared following a previously described protocol. ${ }^{3}$ To reiterate, each of the 20 common proteinogenic amino acids was dissolved in sterile water to a final concentration of $240 \mathrm{mM}$, except for Leu $(200 \mathrm{mM})$. The amino acid solution $(4 \times)$ was prepared on ice by adding $2.5 \mathrm{~mL}$ of water and $125 \mu \mathrm{L}$ of each amino acid in the following order with vigorous mixing after each addition: Ala, Arg, Asn, Asp, Gln, Glu, Gly, His, Ile, Lys, Met, Phe, Pro, Ser, Thr, Val, Trp, Tyr, Leu, Cys. The energy solution (14×) was prepared with the following composition: $700 \mathrm{mM}$ 4-(2-hydroxyethyl)-1-piperazineethanesulfonic acid (HEPES) at pH 8.0, $21 \mathrm{mM}$ ATP, $21 \mathrm{mM}$ GTP, $12.6 \mathrm{mM} \mathrm{CTP}, 12.6 \mathrm{mM} \mathrm{UTP}, 2.8 \mathrm{mg} / \mathrm{mL}$ tRNA (Sigma-Aldrich), $3.64 \mathrm{mM}$ coenzyme A (CoA), $4.62 \mathrm{mM}$ nicotinamide adenine dinucleotide (NAD), $10.5 \mathrm{mM}$ cAMP, $0.95 \mathrm{mM}$ folinic acid, $14 \mathrm{mM}$ spermidine, $420 \mathrm{mM}$ 3-phosphoglyceric acid. Stock solutions of $500 \mathrm{mM}$ magnesium glutamate, $4 \mathrm{M}$ potassium glutamate, and $1 \mathrm{M}$ maltodextrin were also prepared. The energy buffer $(10 \mathrm{~mL})$ was finally prepared by mixing $5.6 \mathrm{~mL}$ amino acid solution $(4 \times), 1.6 \mathrm{~mL}$ energy solution $(14 \times), 400 \mu \mathrm{L}$ magnesium glutamate $(500 \mathrm{mM}), 780 \mu \mathrm{L}$ potassium glutamate $(4 \mathrm{M}), 780 \mu \mathrm{L}$ maltodextrin $(1 \mathrm{M})$ and $840 \mu \mathrm{L}$ water. The resulting energy buffer was vigorously mixed, aliquoted, and stored at $-80{ }^{\circ} \mathrm{C}$ until usage.

DNA template preparation for cell-free biosynthesis (CFB) reactions. Circular plasmids used in CFB were purified with maxi-prep. To be specific, E. coli DH5 $\alpha$ chemically competent cells were transformed with reconstituted plasmid, and the cells were grown overnight on Luria-Bertani (LB) agar plates containing $50 \mu \mathrm{g} / \mathrm{mL}$ kanamycin at $37^{\circ} \mathrm{C}$. Single colonies were picked to inoculate $200 \mathrm{~mL}$ of LB medium containing $50 \mu \mathrm{g} / \mathrm{mL}$ kanamycin and grown at $37^{\circ} \mathrm{C}$ for $16-18 \mathrm{~h}$ with shaking. Cells were harvested via centrifugation at $3,800 \times g$ for $20 \mathrm{~min}$ at $4{ }^{\circ} \mathrm{C}$. The plasmids were isolated and purified using a Qiagen plasmid maxi kit per manufacturer's instructions. The concentration of purified plasmid was determined by measuring the absorbance at $260 \mathrm{~nm}$ using a Nanodrop One $^{\mathrm{C}}$ instrument (Thermo Scientific).

Linear DNA templates used in CFB reactions were amplified through polymerase chain reaction (PCR) from the recombinant pET28 plasmids using primers 44 and 45 (Table S9). The resulting PCR products were purified with Qiagen PCR clean up kit per the manufacturer's instructions. For the linear DNA templates used in library screening, the PCR products were purified with ethanol precipitation (details are described below). The concentrations of purified plasmids were determined using the Nanodrop as above.

Capistruin, burhizin, fusilassin, and cellulassin production by CFB. Reactions were carried out at a total volume of $21 \mu \mathrm{L}$, including: $6.9 \mu \mathrm{L}$ E. coli cell extract, $8.8 \mu \mathrm{L}$ energy buffer, $0.21 \mu \mathrm{L}$ isopropyl $\beta$-D-1-thiogalactopyranoside (IPTG, $50 \mathrm{mM}$ stock concentration) and $0.63 \mu \mathrm{L} \mathrm{DTT}, 100 \mathrm{mM}$ stock concentration). Circular plasmids (20 nM) encoding the biosynthetic gene clusters (BGCs) of capistruin, burhizin, fusilassin (both pET28-MBP-fusA and pACYC-fusBE-fusC were added at $20 \mathrm{nM}$ ), and cellulassin were used to initiate the $\mathrm{CFB}$ reactions. The reactions proceeded at room temperature for 16 
$\mathrm{h}$, then were desalted with ZipTip $\mathrm{C}_{18}$ (Sigma-Aldrich) and eluted with $60 \%$ acetonitrile (ACN). The eluents were analyzed by MALDI-TOF-MS using $\alpha$-cyano-4-hydroxycinnamic acid (CHCA) as the matrix.

High-resolution mass spectrometry and tandem mass spectrometry (HRMS/MS) of lasso peptides. The CFB reactions producing desired lasso peptides were desalted using ZipTip $\mathrm{C}_{18}$ and eluted into $60 \%$ aq. ACN. The eluents were directly infused into a ThermoFisher Scientific Orbitrap Fusion ESI-MS using an Advion TriVersa Nanomate 100. The MS was calibrated and tuned with Pierce LTQ Velos ESI Positive Ion Calibration Solution (ThermoFisher). The MS was operated using the following parameters: resolution, 100,000; isolation width (MS/MS), $1 \mathrm{~m} / \mathrm{z}$; normalized collision energy (MS/MS), 70; activation q value (MS/MS), 0.4; activation time (MS/MS), 30 ms. Fragmentation was performed using collision-induced dissociation (CID) at 35 to $70 \%$. The resulting data were averaged and analyzed using the Qualbrowser application of Xcalibur software (Thermo-Fisher Scientific).

Carboxypeptidase $\mathbf{Y}$ evaluation of threadedness. CFB reactions $(10 \mu \mathrm{L})$ were treated with $60 \%$ ACN. Debris from the quenched reactions was removed by centrifugation at $8,000 \times g$ for $2 \mathrm{~min}$ at room temperature. The supernatant was evaporated to dryness under vacuum and resuspended in $5 \mu \mathrm{L}$ of $50 \mathrm{ng} / \mu \mathrm{L}$ carboxypeptidase $\mathrm{Y}$ in phosphate-buffer saline (PBS). This mixture was briefly mixed by vortex and allowed to react at room temperature for $18 \mathrm{~h}$. Resulting peptidase mixtures were treated with $60 \%$ acetonitrile and analyzed by MALDI-TOF-MS with sinapinic acid (SA) as the matrix. To evaluate heat stability, supernatant obtained after $60 \% \mathrm{ACN}$ treatment were incubated at $95{ }^{\circ} \mathrm{C}$ for $2 \mathrm{~h}$. Heating in the presence of organic solvent was expected to amplify the unfolding pathway for less heat-tolerant lasso peptides. This product was then evaporated to dryness under vacuum and resuspended in $50 \mathrm{ng} / \mu \mathrm{L}$ carboxypeptidase $\mathrm{Y}$ in PBS and reacted at room temperature for $18 \mathrm{~h}$. The digested product was analyzed with MALDI-TOF-MS as above.

Purification of the capistruin standard. A methanol extract of spent media of Burkholderia sp. FERM BP-3421 cultures expressing the capABCD was provided by Prof. Eustáquio (Univ. of Illinois, Chicago $)^{4}$. Capistruin was purified from the extract by high-performance liquid chromatography (HPLC, Agilent 1200 series). Extract was injected onto a C18 column (Thermo Scientific betasil 250 $\times 10 \mathrm{~mm} 5 \mu \mathrm{M}$ particle size). Capistruin was separated using mobile phase A [water, $0.1 \%$ trifluoroacetic acid (TFA)] and mobile phase B (acetonitrile, 0.1\% TFA) over a gradient of 4 min at $10 \% \mathrm{~B}, 2 \min 10-32.5 \% \mathrm{~B}, 8 \min 32.5-35 \% \mathrm{~B}$, and $2 \min 35-95 \%, 4 \min 95 \%$, at a flow rate of 5 $\mathrm{mL} / \mathrm{min}$. UV-vis absorbance from 190-450 nm was recorded. Fractions corresponding to UV absorbance peaks $(220 \mathrm{~nm})$ were collected and tested for the presence of capistruin by MALDI-TOF-MS, using CHCA as the matrix. Capistruin eluted at $14 \mathrm{~min}$; this fraction was concentrated to dryness on the lyophilizer (Labcono FreeZone 6L). The purification was repeated to obtain a $>95 \%$ pure sample of capistruin. A sample of the purified capistruin was resuspended in water at a concentration of $5 \mathrm{mM}$, considered the "standard" sample for our quantification of capistruin by CFB. 
Purification and yield determination of capistruin from CFB. Reactions were carried out at atal volume of $750 \mu \mathrm{L}$ including: $260 \mu \mathrm{L} \mathrm{E}$. coli cell extract, $340 \mu \mathrm{L}$ energy buffer, $24 \mu \mathrm{L}$ DTT (100 mM stock conc.), $9 \mu \mathrm{L}$ IPTG (50 mM stock conc.), $80 \mu \mathrm{L}$ of linear DNA template, and $37 \mu \mathrm{L}$ of water. The linear DNA template was PCR amplified from pET41-capABCD (1400 ng, primers 44 and 45 Table S9), including the $\mathrm{T} 7$ promoter, capABCD, and terminator, was added to initiate the reaction. The reaction was separated into 8 tubes for higher aeration, and incubated at $37{ }^{\circ} \mathrm{C}$ for $4 \mathrm{~h}$, followed by $28{ }^{\circ} \mathrm{C}$ for $20 \mathrm{~h}$ with shaking at $1500 \mathrm{rpm}$. ACN was added to a final concentration of $50 \%$ to precipitate salts and proteins. The reaction was centrifuged at $10,000 \times g$ for $10 \mathrm{~min}$ at room temperature to remove precipitate and the supernatant was concentrated on a lyophilizer.

A standard curve was determined by injections of 3, 7.5, 10, 15, 20 and $30 \mathrm{nmol}$ (approx. 6, 7.5, 10, $15,20,30$, and $60 \mu \mathrm{g}$ ) of the standard sample of capistruin onto the HPLC, under the same conditions. The area of the $220 \mathrm{~nm}$ absorbance peak corresponding to capistruin was calculated for each concentration and plotted together to establish the standard curve $\left(\chi^{2}=0.99\right)$.

Capistruin was purified from the CFB reaction by HPLC in the same manner as the standard sample. The lyophilized CFB reaction was resuspended in $500 \mu \mathrm{L} \mathrm{50 \%} \mathrm{ACN} \mathrm{and} \mathrm{purified} \mathrm{in} \mathrm{a} \mathrm{single} \mathrm{injection.}$ Fractions corresponding to UV absorbance peaks $(220 \mathrm{~nm})$ were collected and tested for the presence of capistruin by MALDI-TOF-MS, using CHCA as the matrix. Capistruin eluted at $14 \mathrm{~min}$, identical retention time to the standard capistruin sample. To determine the yield of capistruin from CFB, the area of the $220 \mathrm{~nm}$ absorbance peak was compared to the standard curve. Purified CFB-produced capistruin was concentrated to dryness on the lyophilizer and stored at $-20{ }^{\circ} \mathrm{C}$ until needed.

Validation of the identity of capistruin purified from CFB by liquid chromatography-mass spectrometry. Capistruin from the CFB reaction was compared to the standard sample by liquid chromatography-mass spectrometry (LC-MS, Shimadzu LCMS 2020). A sample of the purified capistruin from the CFB reaction was injected onto a C18 column (Macherey Nagel EC Nucleodur $250 \times 4.6 \mathrm{~mm} 5 \mu \mathrm{M}$ particle size, $100 \mu \mathrm{L}$ injections), using mobile phase A (water, $0.1 \% \mathrm{FA}$ ) and mobile phase B (ACN, $0.1 \% \mathrm{FA})$ over a gradient of $5 \mathrm{~min}$ at $10 \% \mathrm{~B}, 5 \mathrm{~min} 10-35 \% \mathrm{~B}, 15 \mathrm{~min} 35-65 \%$ $\mathrm{B}$, and $5 \mathrm{~min} 65-95 \%, 4 \mathrm{~min} 95 \%$, at a flow rate of $1 \mathrm{~mL} / \mathrm{min}$. UV-vis absorbance from 190-450 $\mathrm{nm}$ was recorded and mass at $\mathrm{m} / \mathrm{z}$ 500-1500 were monitored. The extracted ion chromatograms of 1025.4 $\mathrm{m} / \mathrm{z}[\mathrm{M}+2 \mathrm{H}]^{2+}$ were compared between capistruin purified from $\mathrm{CFB}$, the standard sample of capistruin, and a co-injection.

Capistruin-dependent inhibition of mCherry production. Capistruin, purified from $750 \mu \mathrm{L}$ of CFB reaction, was resuspended in water to $100 \mu \mathrm{M}$. A CFB reaction was set up with $100 \mathrm{ng}$ plasmid encoding mCherry under T7 control with adding capistruin to a final concentration of 0 or $10 \mu \mathrm{M}$. The reactions were incubated at $30{ }^{\circ} \mathrm{C}$ for $16 \mathrm{~h}$ with $225 \mathrm{rpm}$ shaking for aeration. The samples were centrifuged at $15,000 \times g$ for $1 \mathrm{~min}$ at room temperature to remove precipitate, diluted 1:10 in water, and the fluorescence emission at $600 \mathrm{~nm}$ was quantified (Tecan Infinite M2000) using an excitation wavelength of $550 \mathrm{~nm}(\mathrm{n}=2)$. 
Expression and purification of MBP-tagged FusB, FusE, and FusC. Previously described methods were employed for the expression and purification of MBP-tagged FusB, FusE, and FusC. ${ }^{2}$ Protein concentrations were determined by Bradford colorimetric assay and absorbance at $280 \mathrm{~nm} .{ }^{5}$ Protein purity was visually inspected by SDS-PAGE using Coomassie staining (Figure S11).

Expression and purification of GamS. GamS was purified from E. coli BL21(DE3) bearing the pBAD-gamS plasmid as previously described with minor differences. ${ }^{6}$ An overnight culture was used to inoculate $1 \mathrm{~L}$ of $\mathrm{LB}$ containing $50 \mu \mathrm{g} / \mathrm{mL}$ ampicillin and grown to the $\mathrm{OD}_{600}$ of 0.45 . Arabinose was added to a final concentration of $0.25 \% \mathrm{w} / \mathrm{v}$, followed by an induction period at $37{ }^{\circ} \mathrm{C}$ for $4 \mathrm{~h}$. Cells were harvested via centrifugation at $5,000 \times g$ for $30 \mathrm{~min}$ at $4{ }^{\circ} \mathrm{C}$ and resuspended in $30 \mathrm{~mL}$ lysis buffer [50 mM Tris pH 8.0, $500 \mathrm{mM} \mathrm{NaCl}, 5 \mathrm{mM}$ imidazole, $0.1 \%$ Triton X-100 $(v / v), 3 \mathrm{mg} / \mathrm{mL}$ lysozyme, $2 \mu \mathrm{M}$ leupeptin, $2 \mu \mathrm{M}$ benzamidine $\mathrm{HCl}, 2 \mu \mathrm{M}$ E64, and $30 \mathrm{mM}$ phenylmethylsulfonyl fluoride]. Cells were lysed by sonication for ten cycles (30 s each) at $20 \%$ amplitude, with $30 \mathrm{~s}$ intervals of resting at $4{ }^{\circ} \mathrm{C}$. Insoluble cell debris was removed by centrifugation at $18,000 \times g$ for 60 $\min$ at $4{ }^{\circ} \mathrm{C}$. The resultant supernatant was loaded onto pre-equilibrated nickel-nitrilotriacetic acid (Ni-NTA) resin (ThermoFisher; $5 \mathrm{~mL}$ of resin per L of cells). The column was washed with $30 \mathrm{~mL}$ of wash buffer ( $50 \mathrm{mM}$ Tris $\mathrm{pH} 8.0,500 \mathrm{mM} \mathrm{NaCl}, 25 \mathrm{mM}$ imidazole). The $6 x H$ is-tagged proteins were eluted using $15 \mathrm{~mL}$ elution buffer (50 mM Tris $\mathrm{pH} 8.0,500 \mathrm{mM} \mathrm{NaCl}, 250 \mathrm{mM}$ imidazole). The eluent was concentrated using a $30 \mathrm{kDa}$ molecular weight cut-off Amicon Ultra centrifugal filter (EMD Millipore). A buffer exchange with $10 \times$ volume of protein storage buffer [50 mM Tris-Cl pH 7.5, $100 \mathrm{mM} \mathrm{NaCl}, 1 \mathrm{mM}$ DTT, $1 \mathrm{mM}$ ethylenediaminetetraacetic acid, 2\% dimethyl sulfoxide] was performed prior to final concentration and storage. Protein concentrations were determined by Bradford colorimetric assay and absorbance at $280 \mathrm{~nm}$. Protein purity was visually inspected by SDS-PAGE using Coomassie staining (Figure S11).

Chimeric substrates and FusA Ala variants analysis. The plasmid or linear DNA templates for each mutant and chimeric substrate were prepared as described above and used in a CFB reaction, which was carried out with the total volume of $21 \mu \mathrm{L}$ including $6.9 \mu \mathrm{L}$ E. coli cell extract, $8.8 \mu \mathrm{L}$ energy buffer, $0.21 \mu \mathrm{L}$ IPTG (50 mM initial concentration), $0.63 \mu \mathrm{L}$ DTT (100 mM stock conc.), $0.5 \mu \mathrm{L}$ GamS (125 $\mu$ M stock conc.), $0.5 \mu \mathrm{L}$ FusB (500 $\mu \mathrm{M}$ stock conc.), $1 \mu \mathrm{L}$ FusC (200 $\mu \mathrm{M}$ stock conc.), 0.5 $\mu \mathrm{L}$ FusE (500 $\mu \mathrm{M}$ stock conc.) and $2000 \mathrm{ng}$ plasmid DNA (chimeric substrates) or $500 \mathrm{ng}$ linear DNA (FusA ala variants). The reactions proceeded at room temperature for $16 \mathrm{~h}$ and were quenched by adding ACN to a final concentration of $60 \%$. Debris from the quenched reactions was removed by centrifugation at $8,000 \times g$ for $2 \mathrm{~min}$ at room temperature. The supernatant was analyzed by MALDI-TOF-MS using SA as the matrix.

Linear core peptide detection using the PURExpress system. For each precursor peptide, a $10 \mu \mathrm{L}$ reaction was set up using NEB PURExpress kit per manufacturer instruction s (NEB E6800L) with 2 $\mu \mathrm{L}$ of DNA template added (500 ng for plasmid and $200 \mathrm{ng}$ for linear DNA). The reaction was allowed to proceed at $37{ }^{\circ} \mathrm{C}$ for $3 \mathrm{~h}$. Each heterologously expressed and purified fusilassin protein (20 $\mu \mathrm{M}$ ) was added in $10 \mu \mathrm{L}$ of synthetase buffer (50 mM Tris- $\mathrm{HCl} \mathrm{pH} 7.5,125 \mathrm{mM} \mathrm{NaCl}, 20 \mathrm{mM} \mathrm{MgCl}$, $10 \mathrm{mM}$ DTT, $5 \mathrm{mM}$ ATP), and then added into the PURExpress reactions and incubated for another $3 \mathrm{~h}$ at $37{ }^{\circ} \mathrm{C}$. The reaction was quenched by the addition of ACN directly into the reaction ( $60 \% v / v$ final). 
The debris was removed by centrifugation at $8,000 \times g$ for $2 \mathrm{~min}$ at room temperature prior to analyzing the supernatant by MALDI-TOF-MS with SA as the matrix.

FusA degenerate library screening in 96-well format. DNA containing degenerate segments were synthesized by IDT (Table S3) and amplified through PCR using primers 46 and 47 (Table S9). A linear section of pET28 vector was amplified through PCR using primers 48 and 49 (Table S9), followed by addition of $1 \mu \mathrm{L} \mathrm{DpnI}$ to the reaction and allowed to react at $37{ }^{\circ} \mathrm{C}$ for $1 \mathrm{~h}$. The PCR amplicons were purified using a QIAprep PCR Cleanup Spin Kit. The isolated FusA degenerate library and the linear section of pET28 plasmid was then simultaneously digested with KpnI and SacI. Digested insert and vector were then purified with QIAprep PCR Cleanup Spin Kit (Qiagen) and ligated using T4 DNA ligase (NEB). The ligation reactions were directly transformed into high efficiency, chemically competent E. coli DH5 a (NEB 5-alpha Competent E. coli). The cells were plated on $4 \mathrm{LB}$ agar plates $(10 \mathrm{~cm})$ containing $50 \mu \mathrm{g} / \mathrm{mL}$ kanamycin. The plates were incubated at $37{ }^{\circ} \mathrm{C}$ for $18 \mathrm{~h}$. The PCR mixture was prepared using primers 44 and 45 (Table S9) and aliquoted into the 96-well plate with $50 \mu \mathrm{L}$ per well. Single colonies $(\mathrm{n}=96)$ were picked and added into each well. The PCR products were purified by addition of $100 \mu \mathrm{L}$ ethanol and $5 \mu \mathrm{L}$ sodium acetate (3 M, pH 5.2) to each well followed by DNA precipitation at $-20^{\circ} \mathrm{C}$ for $1 \mathrm{~h}$. After DNA precipitation, the plate was subjected to centrifugation at $4,500 \times g$ for $30 \mathrm{~min}$ at $4{ }^{\circ} \mathrm{C}$ to harvest the DNA product. The supernatant was removed, and the DNA precipitate was resuspended in $70 \%$ ethanol for an additional washing step. Supernatant was removed by centrifugation at $4,500 \times g$ for $30 \mathrm{~min}$ at $4{ }^{\circ} \mathrm{C}$. The DNA precipitate was air-dried and dissolved in $25 \mu \mathrm{L}$ of water.

The CFB reaction mixture was prepared with $180 \mu \mathrm{L}$ E. coli cell extract, $141 \mu \mathrm{L}$ energy buffer, 12.6 $\mu \mathrm{L}$ DTT (100 mM stock conc.), $4.2 \mu \mathrm{L} \mathrm{IPTG} \mathrm{(50} \mathrm{mM} \mathrm{stock} \mathrm{conc.),} 10 \mu \mathrm{L} \mathrm{GamS} \mathrm{(125} \mu \mathrm{M}$ stock conc.), $10 \mu \mathrm{L}$ MBP-tagged FusB (500 $\mu \mathrm{M}$ stock conc.), $10 \mu \mathrm{L}$ MBP-tagged FusC (500 $\mu \mathrm{M}$ stock conc.) and 10 $\mu \mathrm{L}$ MBP-tagged FusE (500 $\mu \mathrm{M}$ stock conc.). The reaction mixture was then aliquoted into the 96 -well plate with $3.6 \mu \mathrm{L}$ in each well. Linear DNA template $(1 \mu \mathrm{L}, \sim 200 \mathrm{ng})$ was then added individually to initiate the CFB reaction. Reactions were quenched after incubating of $16 \mathrm{~h}$ at room temperature by addition of $5.4 \mu \mathrm{L} \mathrm{ACN}$ to each well $(60 \% v / v)$. The plate was then subjected to centrifugation at 4,500 $\times g$ for $15 \mathrm{~min}$ at $4{ }^{\circ} \mathrm{C}$. The supernatant was analyzed by MALDI-TOF-MS with SA as the matrix.

For multiple FusA variants produced in a single reaction, each CFB reaction containing one FusA variant was initiated as described above. Afterward, 6 reactions were pooled, allowed to react at room temperature for $16 \mathrm{~h}$, and then analyzed by MALDI-TOF-MS as above.

Statistical estimation of mature lasso peptides possible from the ring library. The normal approximate interval was deemed appropriate for providing an estimate for how many mature lasso peptides would result from the 3.2 M-membered ring library. Given the number of successes and failures were known for the sample size, the binomial proportion confidence interval was applicable. Also, the sample size was sufficiently large and the success probability was not very close to 0 or 1 , allowing the use of the Wald method with the following equation. ${ }^{7}$

$$
\frac{W}{2}=Z \sqrt{\frac{p(1-p)}{n}}
$$


$\mathrm{W}$ represents the total width of the distribution (therefore $\mathrm{W} / 2$ is the margin of error), $\mathrm{Z}$ is the $\mathrm{Z}$-score, and $\mathrm{p}$ is the proportion of the sample population that was sampled (in our case, successfully cyclized). We used a population Z-score of 1.96, which equates to the $95 \%$ confidence interval, giving p as 52\%. $\mathrm{W} / 2$ is then calculated at $3.12 \%$. Therefore, with $95 \%$ confidence, we can say that FusC is capable of producing 1.85-2.05 $\mathrm{M}$ lasso peptides from our 3.2 M-membered ring library.

Heatmap analysis for the amino acid occurrence at each position. The observed occurrence $\left(\mathrm{O}_{\mathrm{obs}}\right)$ of each residue at each position was calculated based on the sequences of FusC substrates or non-substrates. The predicted occurrence $\left(\mathrm{O}_{\text {pre }}\right)$ of each residue based on the degenerate NNK codon (N=A, T, C, G; K = T, G) was as follows: Glu: 3.1\%; Gly: 6.3\%; Asp: 3.1\%; Met: 3.1\%; Ala: 6.3\%; Val: 6.3\%; Asn: 3.1\%; Lys: 3.1\%; Thr: 6.3\%; Ile: 3.1\%; Gln: 3.1\%; Arg: 9.4\%; His: $3.1 \%$; Phe: $3.1 \%$; Ser: 9.4\%; Leu: 9.4\%; Tyr: 3.1\%; Trp: $3.1 \%$; Cys: $3.1 \%$; Pro: $6.3 \%$. The percent difference (D\%) between the two was then calculated using:

$$
\begin{aligned}
& \mathrm{D} \%=\left(O_{o b s}-O_{p r e}\right) / O_{p r e} * 100 \% \text { if } O_{o b s} \text { is higher than } O_{\text {pre }} \\
& \mathrm{D} \%=\left(O_{o b s}-O_{\text {pre }}\right) / O_{o b s} * 100 \% \text { if } O_{o b s} \text { is lower than } O_{\text {pre }}
\end{aligned}
$$

Calculation of percent surface area of lasso peptide ring region. For each lasso peptide structure with a PDB file, the exposed surface area for each residue was calculated in UCSF Chimera. ${ }^{8}$ The surface command for each strand calculated the solvent-excluded molecular surface. The solvent accessible surface area (SASA) was calculated by residue. ${ }^{9}$ The SASA for the ring region was the sum of the residues in the macrocycle, while the SASA of the loop/tail was the sum of the remaining residues (Table $\mathbf{S 4}$ ).

Compilation of all predicted lasso peptides found in NCBI. A diverse set of known lasso leader peptidases (Table S6) were used as queries for a four-iteration Position-Specific Iterated BLAST (PSI- BLAST ${ }^{10}$ ) search (November 2020). Default settings were used except the maximum target sequence was increased to 20,000. The eleven searches were combined for a non-redundant list of 9,571 protein accession identifiers from Protein Family ${ }^{11}$ PF13471 (transglut_core3) within the NCBI database. Each NCBI protein accession identifier was submitted to RODEO (Rapid ORF Description $\&$ Evaluation Online) using the lasso peptide scoring function. ${ }^{12}$ Initial analysis yielded $\sim 452,000$ putative open reading frames co-occurring with the queried leader peptidase. Hypothetical peptides receiving a RODEO score $<5$ were disqualified from further analysis. Peptides receiving a score of 5 or greater $(\sim 38,500)$ are provided in Dataset 1. Peptides receiving a score of 10 or greater were considered lasso precursor peptides. Leader peptidase queries that did not co-occur with a putative lasso cyclase (PF00733, asparagine synthetase homologs) were also removed. Entries with the same leader peptidase query, where the ending nucleotide position was the same, but the starting positions were variable due to ambiguity over start codons, were considered identical. The longest of these entries with the highest RODEO lasso score was retained while the remaining were removed such that each unique core peptide sequence (same stop codon) occurred only once per leader peptidase query, resulting in $\sim 8,000$ precursor peptides. The ring size and acceptor residue were predicted and manually inspected. Entries with core sequences lacking a single Asp or Glu within position 7-16 were considered invalid and removed. In total, 7,701 total peptides were identified in the NBCI 
database that were encoded in a suspected lasso peptide BGC that appeared to be competent substrates.

Bioinformatic analysis of naturally encoded lasso core peptides. To reveal sequence trends in the core region of predicted lasso peptide precursors, any database entries $(n=7,701)$ with identical core sequences were removed, yielding 4,485 unique predicted core sequences. The acceptor site was determined as either the Asp or Glu that was in the $7^{\text {th }}, 8^{\text {th }}$, or $9^{\text {th }}$ core position. If multiple Asp or Glu were found at positions 7-9 for uncharacterized lasso peptide families, the acceptor site was presumed to yield the larger macrocycle, which was based on a recent statistical analysis of naturally encoded lasso precursor peptides $\left(5.5 \%, 36.6 \%\right.$, and $57.9 \%$ for 7 -mers, 8 -mers, and 9-mers, respectively). ${ }^{2}$ The only exception was if Glu was found at both positions 7 and 8, the acceptor site was presumed to be at position $7(n=170 / 7701,2.2 \%)$, similar to the observed structure of the xanthomonins. ${ }^{13}$ If there was neither Asp or Glu at position 7-9, the acceptor residue was assigned as the next-closest Asp or Glu to the N-terminal of the core peptide beyond position $9(10$-mers $n=505 / 7701,6.6 \%, 11+$-mers $n=$ $318 / 7701,4.1 \%)$.

The variability of the residues was calculated with non-redundant core sequences, either only sequences predicted to have a 9-residue macrolactam or 9-residue macrolactam with a Glu-acceptor (same macrocycle as fusilassin). Three different variability measurements were calculated: Shannon entropy ${ }^{14}$ consurf, ${ }^{15}$ and relative entropy (Table S7). ${ }^{16}$ The frequency of each amino acid at each position of the lasso peptides was then determined with the non-redundant core sequences. For analysis, only 9-mer predicted lasso peptides were used, the same macrocycle size as fusilassin. The frequency of each amino acid was compared to its expected frequency. The expected frequency was chosen to be the weighted average codon frequency for the most represented genus (Table S8) ${ }^{17}$. 
Table S1. Genetically predicted lasso peptides used for FusA chimeric precursor generation. The leader region of FusA was retained while the core sequences of 10 alternative precursor peptides were used (acceptor residue is red) to evaluate FusB/C/E compatibility. The FusA core sequence is aligned to each entry with bold and underlined representing identical and similar residues. Hyphens represent gaps in the alignment. Values in parenthesis indicate percent identity/similarity (lenient). Percent identity (\%ID) of the lasso cyclase present in the alternative BGC is also compared with FusC (WP_104612995.1).

\begin{tabular}{|c|c|c|c|c|}
\hline Name & $\begin{array}{l}\text { NCBI accession } \\
\text { (lasso cyclase) }\end{array}$ & Bacterial strain & $\begin{array}{l}\text { Core sequence (top) } \\
\text { FusA core sequence (bottom) }\end{array}$ & $\begin{array}{c}\text { \%ID to } \\
\text { FusC }\end{array}$ \\
\hline CelA & WP_068757176.1 & $\begin{array}{l}\text { Thermobifida cellulosilytica } \\
\text { TB100 }\end{array}$ & $\begin{array}{l}\text { WIQGKWGLEIYLIFPRYL } \\
\text { WYTAEWGLELIFVFPRFI }\end{array}$ & 77 \\
\hline HalA & WP_068689552.1 & Thermobifida halotolerans & 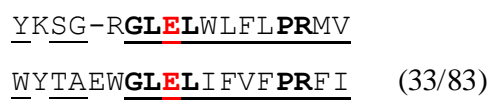 & 71 \\
\hline MthA & WP_078762521.1 & $\begin{array}{l}\text { Marinactinospora } \\
\text { thermotolerans DSM } 45154\end{array}$ & $\begin{array}{l}\underline{Y}-\underline{N} I N K \underline{\underline{L}} \text { IIFIWPRLFN } \\
\underline{\text { WYTAEWGLELIFVFPRFI }}\end{array}$ & 55 \\
\hline $\mathrm{NcaA}$ & WP_052423756.1 & Nonomuraea candida & $\begin{array}{l}\text { YV-GLRNRESLLGYPRN IW } \\
\underline{\text { WYTAEWGLELI FVE PRF I }}\end{array}$ & 49 \\
\hline MobA & WP_051072671.1 & $\begin{array}{l}\text { Streptomyces mobaraensis } \\
\text { DSM } 40847\end{array}$ & 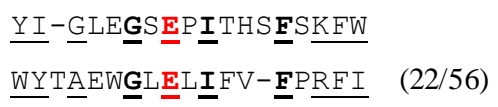 & 49 \\
\hline RubA & WP_045701015.1 & Streptomyces rubellomurinus & $\begin{array}{l}\text { AL-GLHGAEPFFPTLHTSWW } \\
\text { WYTAEWGLELIFVFPRFI }\end{array}$ & 45 \\
\hline NbsA & WP_089507158.1 & Streptomyces sp. NBS 14/10 & 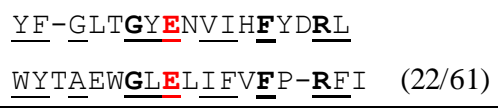 & 44 \\
\hline SruA & WP_107644840.1 & Streptomyces sp. Ru87 & $\begin{array}{l}\text { RG-GEP IWEEVVVPWDYWV } \\
\text { WYTAEWGLELIFVFPRFI }\end{array}$ & 44 \\
\hline SleA & WP_048574008.1 & Streptomyces leeuwenhoekii & 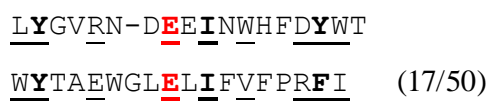 & 42 \\
\hline AreA & WP_048574008.1 & Actinoplanes regularis & $\begin{array}{ll}\text { MY } \underline{\mathbf{Y}} \text { GRRGYERTLQT-KA } & \left(\mathrm{D} 8 \mathrm{E}^{1}\right) \\
\text { WYTAEWGLELIFVFPRFI } & (17 / 50)\end{array}$ & 41 \\
\hline $\mathrm{CitA}^{2}$ & WP_038518372.1 & Streptomyces albulus & $\begin{array}{ll}\text { LLGLA-GNERT LVLSKN } & \left(\mathrm{D} 8 \mathrm{E}^{1}\right) \\
\underline{\text { WYTAEWGLELI FVE PRF I }} & (11 / 39)\end{array}$ & 38 \\
\hline
\end{tabular}

${ }^{1}$ The acceptor residues of AreA and CitA were changed from Asp to Glu.

${ }^{2} \mathrm{Cit} \mathrm{A}$ is the precursor peptide of citrulassin A, which was reported previously ${ }^{2}$ and not included in this work. 
Table S2. Nucleotide sequences of chimeric precursor peptides. The codons are optimized for $E$. coli. All sequences are provided 5' to $3^{\prime}$. Restriction sites (BamHI, HindIII) are underlined. LP, leader peptide; $\mathrm{CP}$, core peptide.

FUSA $_{\mathrm{LP}}-$ CelA $_{\mathrm{CP}}$

GGATCCATGGAAAAGAAGAAGTACACGGCCCCTCAGCTGGCTAAGGTAGGCGAATTTAAGGAAGCCACTGGATGGATTCAAGG CAAATGGGGCCTGGAGATTTACCTGATCTTCCCGCGCTATTTGTAGAAGCTT

FUSA $_{\mathrm{LP}}-$ HalA $_{\mathrm{CP}}$

GGATCCATGGAAAAGAAGAAGTACACGGCCCCACAACTGGCCAAGGT TGGTGAGTTTAAGGAAGCGACTGGTTACAAGTCGGG TCGTGGTTTAGAGCTTTGGCTGTTTCTCCCCCGTATGGTATAAAAGCTT

FusA $A_{\mathrm{LP}}-M t h A_{\mathrm{CP}}$

GGATCCATGGAAAAGAA GAAGTACACGGCCCCTCAACTGGCCAAGGT TGGCGAGTTTAAGGAAGCAACAGGTTACAATGCCAT CAATAAGTTAGAGATCATCTTCATCTGGCCTCGTTTATTCAATTAAAAGCTT

FusA $_{\mathrm{LP}}-\mathrm{NcaA} \boldsymbol{A}_{\mathrm{CP}}$

GGATCCATGGAAAAGAAGAAGTACACGGCCCCTCAATTGGCAAAGGT GGGTGAGTTTAAGGAAGCGACAGGTTACGTCGGATT GCGTAATCGTGAGTCGCTTTTAGGGTACCCCCGTAATATCTGGTAAAAGCTT

FUSA $A_{\mathrm{LP}}-\mathrm{MobA}_{\mathrm{CP}}$

GGATCCATGGAAAAGAAGAAGTACACGGCACCCCAACTCGCTAAGGTGGGCGAGTTTAAGGAAGCGACGGGATACATCGGTCT CGAGGGAAGCGAGCCTATCACTCACTCGTTCTCCAAGTTCTGGTAAAAGCTT

\section{Fus $_{\mathrm{LP}}-\boldsymbol{R u b A _ { \mathrm { CP } }}$}

GGATCCATGGAAAAGAAGAAGTACACGGCACCACAACTCGCTAAGGTAGGTGAGTTTAAGGAAGCGACGGGTGCCTTAGGACT

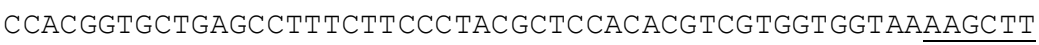

FUSA $A_{\mathrm{LP}}-N b s A_{\mathrm{CP}}$

GGATCCATGGAAAAGAAGAAGTACACT GCACCTCAATTGGCCAAGGT GGGCGAGTTTAAGGAAGCGACTGGGTACTTCGGTTT GACTGGGTACGAGAATGTAATCCACTTCTACGACCGTCTCTAAAAGCTT

FusA $_{\mathrm{LP}}-\mathrm{SruA}_{\mathrm{CP}}$

GGATCCATGGAAAAGAAGAAGTACACT GCCCCACAATTGGCAAAGGT GGGTGAGTTTAAGGAAGCAACGGGTCGTGGTGGTGA GCCCATCTGGGAAGAGGTTGTCGTCCCATGGGACTACTGGGTTTAAAAGCTT

FUSA $_{\mathrm{LP}}-$ SleA $A_{\mathrm{CP}}$

GGATCCATGGAAAAGAAGAAGTACACAGCACCTCAACTCGCCAAGGTAGGTGAGTTTAAGGAAGCGACTGGTTTATACGGTGT CCGTAATGACGAGGAGATCAATTGGCACTTCGACTACTGGACTTAAAAGCTT

\section{FusA $_{\mathrm{LP}}-$ AreA $_{\mathrm{CP}}(\mathrm{D} 8 \mathrm{E})$}

GGATCCATGGAAAAGAAGAAGTACACGGCACCTCAACTCGCAAAGGT TGGCGAGTTTAAGGAAGCGACGGGTATGTACGGACG TCGTGGTTACGAACGTACGCTCCAAACGAAGGCCTGAAAGCTT 
Table S3. Nucleotide sequences of FusA degenerate libraries. All sequences are provided 5' to 3' direction. Top, "Ring" library with the NNK (orange) region located in the ring region of FusA. Bottom, "Loop" library with NNK codons appearing in the loop region of FusA. Restriction sites (KpnI and SacI) are underlined. Color-coding: blue, T7 promotor/terminator; purple, lac operator; red, ribosome-binding sequence. The FusA coding sequence is in bold.

FusA ring degenerate library:

GGTACCATCCCGCGAAATTAATACGACTCACTATAGGGGAATTGTGAGCGGATAACAATTCCCCTCTA GAAATAATTTTGTTTAACTTTAAGAAGGAGATATACCATGGAAAAGAAGAAGTACACCGCTCCGCAGC TCGCTAAGGTCGGCGAATTCAAGGAGGCCACCGGCTGGNNKNNKNNKNNKNNKGGCCTCGAGCTGATC TTCGTCTTCCCGCGCTTCATCTGAAGGAAGCTGAGTTGGCTGCTGCCACCGCTGAGCAATAACTAGCA TAACCCCTTGGGGCCTCTAAACGGGTCTTGAGGGGTTTTTTGGAGCTC

FusA loop degenerate library:

GGTACCATCCCGCGAAATTAATACGACTCACTATAGGGGAATTGTGAGCGGATAACAATTCCCCTCTA GAAATAATTTTGTTTAACTTTAAGAAGGAGATATACCATGGAAAAGAAGAAGTACACCGCTCCGCAGC TCGCTAAGGTCGGCGAATTCAAGGAGGCCACCGGCTGGTACACCGCGGAATGGGGCCTCGAGNNKNNK NNKNNKNNKCCGCGCTTCATCTGAAGGAAGCTGAGTTGGCTGCTGCCACCGCTGAGCAATAACTAGCA TAACCCCTTGGGGCCTCTAAACGGGTCTTGAGGGGTTTTTGGAGCTC 
Table S4. Expected surface area for ring region among available lasso peptide structures in PDB. The surface areas for 21 lasso peptides were analyzed and the estimated surface areas for both ring and loop + tail were calculated. Blue, ring region; red, acceptor residue. Underlined residues are not shown in the crystal structure.

\begin{tabular}{|c|c|c|c|c|c|}
\hline Name & Core sequences & $\begin{array}{l}\text { PDB } \\
\text { entry }\end{array}$ & $\begin{array}{c}\text { Estimated } \\
\text { surface area } \\
(\text { ring })\left(\AA^{2}\right)\end{array}$ & $\begin{array}{c}\text { Estimated } \\
\text { surface area } \\
(\text { loop+tail })\left(\AA^{2}\right)\end{array}$ & $\begin{array}{c}\% \text { estimated } \\
\text { surface area } \\
\text { (ring) }\end{array}$ \\
\hline microcin $\mathrm{J} 25$ & GGAGHVPEYFVGIGTPISFYG & $1 \mathrm{Q} 71$ & 561 & 1170 & $32 \%$ \\
\hline astexin-1 & GLSQGVEPDIGQTYFEESRINQD & 2LTI & 632 & 1231 & $34 \%$ \\
\hline caulosegnin I & GAF'VGQPEAVN PLGREIQG & 2LX6 & 647 & 1132 & $36 \%$ \\
\hline astexin-3 & GPTPMVGLDSVSGQYWDQHAPLAD & $2 \mathrm{M} 8 \mathrm{~F}$ & 576 & 1461 & $28 \%$ \\
\hline lassomycin & GLRRLFADQLVGRRNI & 2MAI & 771 & 1063 & $42 \%$ \\
\hline xanthomonin II & GGPLAGEEMGG ITT & $2 \mathrm{MFV}$ & 519 & 752 & $41 \%$ \\
\hline caulonodin V & S IGD SGLRE SMSSQTYWP & $2 \mathrm{MLJ}$ & 800 & 922 & $46 \%$ \\
\hline streptomonomicin & SLGSSPYNDILGYPALIVIYP & 2MW3 & 736 & 1320 & $36 \%$ \\
\hline citrocin & GGVGKIIEYFI GGGVGRYG & 6MW6 & 633 & 980 & $39 \%$ \\
\hline chaxapeptin & GFGSKPLDSFGLNFF & $2 \mathrm{~N} 5 \mathrm{C}$ & 703 & 818 & $46 \%$ \\
\hline astexin-2 & GLTQIQALD SVSGQFRDQLG & $2 \mathrm{~N} 6 \mathrm{U}$ & 828 & 1153 & $42 \%$ \\
\hline xanthomonin I & GGPLAGEE I GG FNVPG I SEE & $4 \mathrm{NAG}$ & 335 & 774 & $30 \%$ \\
\hline caulosegnin II & GTLTPGLPEDF LPGHYMPG & 5D9E & 714 & 1390 & $34 \%$ \\
\hline sphaericin & GLPI GWWIERP SGWYFPI & $5 \mathrm{GVO}$ & 857 & 1027 & $45 \%$ \\
\hline sphingopyxin I & GIEPLGPVDEDQGEHYLFAGG & $5 \mathrm{JQF}$ & 543 & 839 & $39 \%$ \\
\hline rubrivinodin & GAPS LINSEDN PAFPQRV & $50 Q Z$ & 698 & 909 & $43 \%$ \\
\hline benenodin-1 & GVGF GRPDS ILTQE QAK PMGLDRD & $5 \mathrm{TJ} 1$ & 608 & 1051 & $37 \%$ \\
\hline acinetodin & GGKGPIFETWVTEGNYYG & 5UI6 & 620 & 962 & $39 \%$ \\
\hline klebsidin & GSDGPIIEFFNPNGVMHYG & $5 \mathrm{UI7}$ & 592 & 1120 & $35 \%$ \\
\hline subterisin & GPPGDRIEFGVLAQLPGLDRD & 5XM4 & 722 & 908 & $44 \%$ \\
\hline ubonodin & GGDGSIAEY FNRPMH I HDWQ IMDS GYYG & 6POR & 516 & 2184 & $19 \%$ \\
\hline
\end{tabular}

average $=38 \%$ 
Table S5. List of sequence-confirmed ring variants with mature lasso peptide formation. The fusilassin ring variants that were successfully converted into mature lasso peptides contain residue substitutions within positions 2-6 of the FusA core sequence. The number of fusilassin variants with 1-5 substitutions are summarized and tabulated. Wild type residues are highlighted in red.

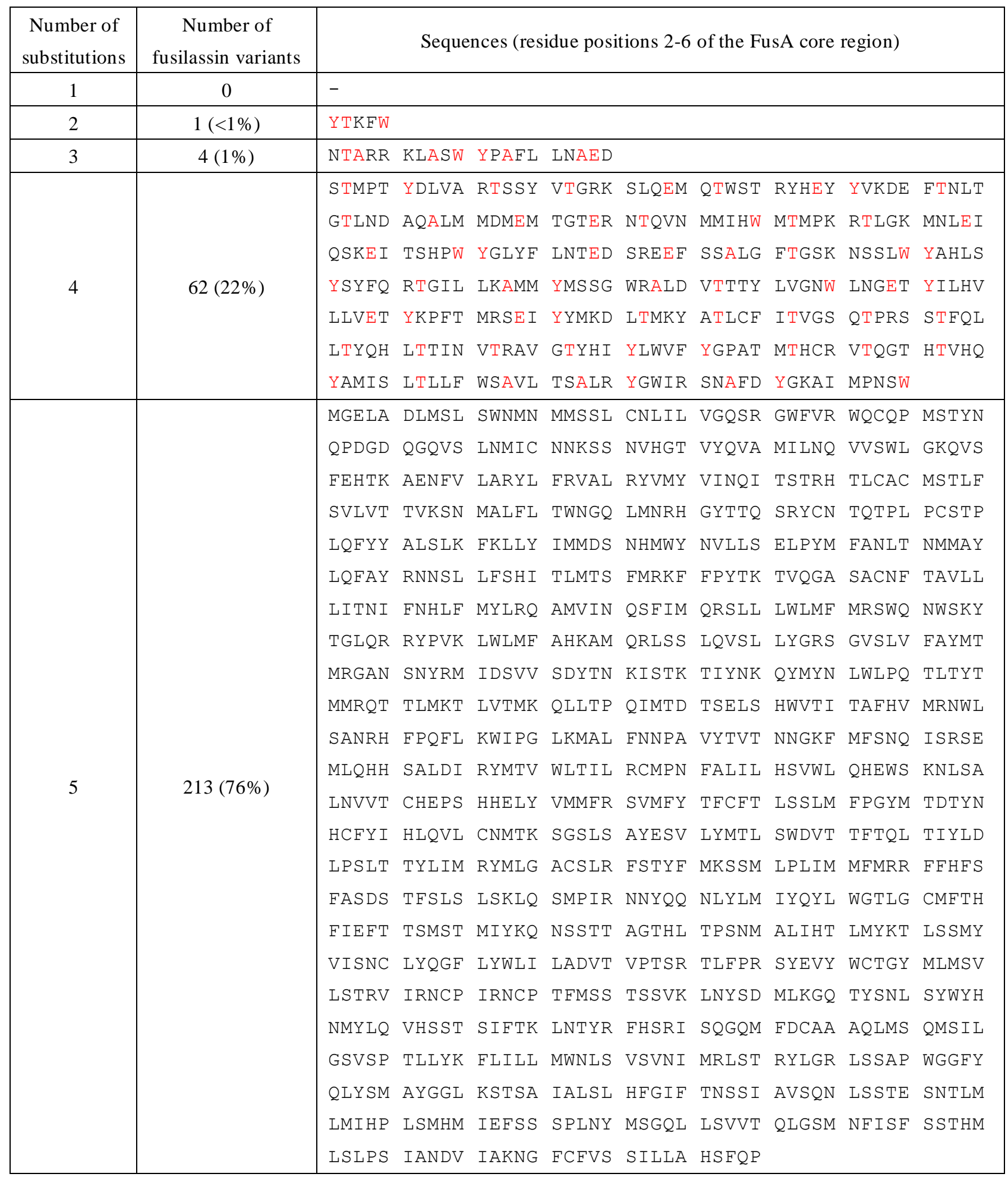


Table S6. Protein accession identifiers used to mine lasso peptide BGCs from NCBI. Each protein is lasso leader peptidase (PF13471) from a known lasso peptide biosynthetic gene cluster (BGC).

\begin{tabular}{|c|c|c|c|c|c|}
\hline Lasso peptide & Genus and species & Phylum & Class & Linkage & NCBI accession \\
\hline citrulassin $\mathrm{A}$ & Streptomyces albus & Actinobacteria & II & Leu-Asp 8mer & WP_030547855.1 \\
\hline specialicin & Streptomyces specialis & Actinobacteria & I & Cys-Asp 9mer & WP_059005882.1 \\
\hline xanthomonin III & Xanthomonas citri & Proteobacteria & II & Gly-Glu 7 mer & WP_050545040.1 \\
\hline pseudomycoidin & Bacillus pseudomycoides & Firmicutes & II & Ala-Asp 9mer & EEM14410.1 \\
\hline caulonodin VI & Caulobacter sp. & Proteobacteria & II & Ala-Glu 9mer & WP_049771798.1 \\
\hline LP2006 & Nocardiopsis alba & Actinobacteria & IV & Gly-Glu 8mer & WP_014912067.1 \\
\hline pandonodin & $\begin{array}{l}\text { Pandoraea } \\
\text { norimbergensis }\end{array}$ & Proteobacteria & IV & Gly-Glu 8mer & WP_058377878.1 \\
\hline 9401-LP1 & Streptomyces sp. & Actinobacteria & III & Ala-Asp 9mer & ARA91542.1 \\
\hline paeninodin & $\begin{array}{l}\text { Paenibacillus } \\
\text { dendritiformis }\end{array}$ & Firmicutes & II & Ala-Asp 9mer & EHQ60563.1 \\
\hline capistruin & $\begin{array}{l}\text { Burkholderia } \\
\text { thailandensis }\end{array}$ & Proteobacteria & II & Gly-Asp 9mer & ABC38564.1 \\
\hline lariatin $\mathrm{B}$ & Rhodococcus jostii & Actinobacteria & II & Gly-Glu 8mer & BAL72549.1 \\
\hline
\end{tabular}


Table S7. Natural variability in the set of genomically predicted lasso peptides. The set has been reduced to only include one entry for each unique core sequence $(n=4,485)$. The position with the greatest variability per metric is highlighted in red while the next three most variable positions are in bold. Acceptor residues were predicted based on the position of the Asp and/or Glu residues as described in the Methods. White background indicates ring region while gray background indicates loop/tail region.

\begin{tabular}{|c|c|c|c|c|c|c|}
\hline \multirow{2}{*}{$\begin{array}{c}\text { Core } \\
\text { position }\end{array}$} & \multicolumn{2}{|c|}{ 9-residue ring, Asp- and Glu-acceptor } & \multicolumn{2}{c|}{ 9-residue ring, Glu only-acceptor } \\
\cline { 2 - 7 } & $\begin{array}{c}\text { Shannon } \\
\text { entropy }\end{array}$ & $\begin{array}{c}\text { Consurf } \\
\mathbf{( 1 - 9 )}\end{array}$ & $\begin{array}{c}\text { Relative } \\
\text { entropy }\end{array}$ & $\begin{array}{c}\text { Shannon } \\
\text { entropy }\end{array}$ & $\begin{array}{c}\text { Consurf } \\
\text { (1-9) }\end{array}$ & $\begin{array}{c}\text { Relative } \\
\text { entropy }\end{array}$ \\
\hline 1 & 2.91 & 6.5 & 0.155 & 3.05 & 7 & 0.156 \\
\hline 2 & 3.74 & 5.5 & 0.074 & 4.02 & 5.5 & 0.054 \\
\hline 3 & 3.91 & $\mathbf{4 . 5}$ & 0.044 & 4.03 & 5.5 & $\mathbf{0 . 0 2 0}$ \\
\hline 4 & 3.87 & $\mathbf{4 . 5}$ & 0.044 & 4.00 & $\mathbf{4}$ & $\mathbf{0 . 0 2 6}$ \\
\hline 5 & 3.85 & $\mathbf{4 . 5}$ & 0.046 & 3.84 & 5 & 0.036 \\
\hline 6 & 3.94 & $\mathbf{4 . 5}$ & 0.037 & 3.96 & $\mathbf{5 . 5}$ & 0.037 \\
\hline 7 & 4.21 & $\mathbf{3 . 5}$ & $\mathbf{0 . 0 2 5}$ & $\mathbf{4 . 1 4}$ & $\mathbf{3}$ & 0.036 \\
\hline 8 & 3.99 & 5.5 & 0.204 & 3.97 & 5.5 & 0.269 \\
\hline 9 & 0.89 & 9 & 0.386 & 0 & 9 & 0.489 \\
\hline 10 & 4.10 & 5.5 & 0.201 & $\mathbf{4 . 1 2}$ & 5.5 & 0.248 \\
\hline 11 & 4.01 & $\mathbf{4 . 5}$ & $\mathbf{0 . 0 2 7}$ & 4.06 & 5 & $\mathbf{0 . 0 2 3}$ \\
\hline 12 & 3.97 & 5.5 & 0.038 & 4.04 & 5.5 & 0.029 \\
\hline 13 & 4.07 & $\mathbf{4 . 5}$ & 0.035 & $\mathbf{4 . 1 7}$ & 5.5 & $\mathbf{0 . 0 2 8}$ \\
\hline 14 & $\mathbf{4 . 1 2}$ & 5.5 & $\mathbf{0 . 0 3 0}$ & 4.10 & 5.5 & 0.029 \\
\hline 15 & $\mathbf{4 . 1 5}$ & 5.5 & $\mathbf{0 . 0 3 1}$ & $\mathbf{4 . 1 1}$ & 5.5 & 0.040 \\
\hline 16 & $\mathbf{4 . 2 0}$ & $\mathbf{4 . 5}$ & 0.035 & $\mathbf{4 . 1 1}$ & $\mathbf{4 . 5}$ & 0.057 \\
\hline
\end{tabular}


Table S8. Determination of the "expected frequency" as a weighted average of the most frequent genus to have a predicted lasso peptide gene cluster. The genus, number of CDSs included in the analysis, the residue frequency and the weight for the average, is shown. The determined frequency is Glu: $6.0 \%$; Gly: $8.2 \%$; Asp: $5.7 \%$; Met: 2.2\%; Ala: 11.7\%; Val: 7.6\%; Asn: 2.9\%; Lys: 3.9\%; Thr: 5.6\%; Ile: 5.1\%; Gln: 3.3\%; Arg: 6.8\%; His: 2.1\%; Phe: 3.6\%; Ser: 5.5\%; Leu: 9.8\%; Tyr: 2.6\%; Trp: 1.4\%; Cys: $0.8 \%$; Pro: $5.2 \%$. All the values shown in the table are percentages

\begin{tabular}{|c|c|c|c|c|c|c|c|c|c|c|c|c|c|c|c|c|c|c|c|}
\hline 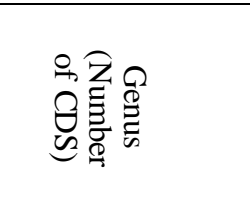 & 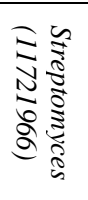 & 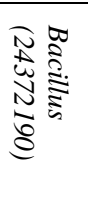 & 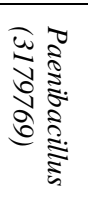 & 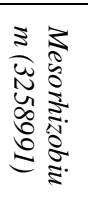 & 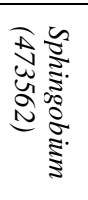 & 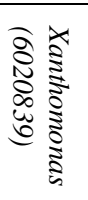 & 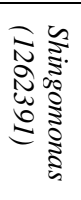 & 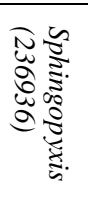 & 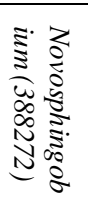 & 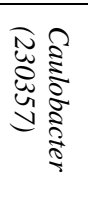 & 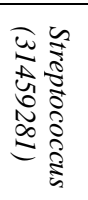 & 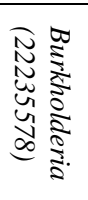 & 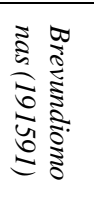 & 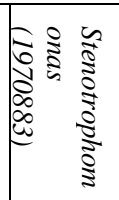 & 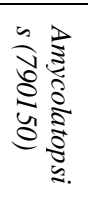 & 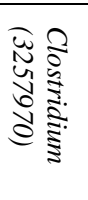 & 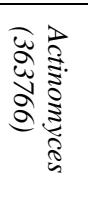 & 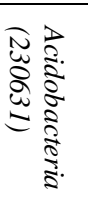 & 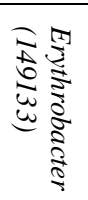 \\
\hline $\mathrm{E}$ & 5.7 & 7.4 & 6.7 & 5.5 & 5.2 & 4.8 & 5.7 & 5.3 & 5.3 & 5.3 & 7.1 & 4.7 & 5.6 & 5.1 & 5.7 & 7.6 & 5.8 & 5.5 & 6.5 \\
\hline $\mathrm{G}$ & 9.6 & 6.8 & 7.5 & 8.6 & 8.8 & 8.2 & 3.6 & 9.0 & 8.9 & 9.0 & 6.6 & 8.3 & 9.0 & 8.6 & 9.3 & 4.7 & 9.0 & 8.1 & 8.8 \\
\hline $\mathrm{D}$ & 5.9 & 4.8 & 5.1 & 5.7 & 6.1 & 5.7 & 6.7 & 6.2 & 5.8 & 5.9 & 5.6 & 5.7 & 6.0 & 5.7 & 5.9 & 5.6 & 5.9 & 5.1 & 6.2 \\
\hline $\mathrm{M}$ & 1.6 & 2.7 & 2.8 & 2.4 & 2.5 & 2.1 & 2.5 & 2.4 & 2.4 & 2.1 & 2.5 & 2.1 & 2.3 & 2.1 & 1.5 & 2.7 & 2.0 & 2.2 & 2.4 \\
\hline A & 13.7 & 6.9 & 8.2 & 12.4 & 13.1 & 13 & 15.4 & 13.6 & 13.3 & 13.9 & 7.5 & 14.2 & 13.8 & 12.9 & 13.3 & 5.9 & 13.2 & 11 & 12.6 \\
\hline $\mathrm{V}$ & 8.4 & 7.1 & 7.0 & 7.4 & 6.9 & 7.3 & 8.2 & 7.0 & 7.2 & 7.6 & 7.0 & 7.5 & 7.6 & 7.4 & 8.9 & 6.5 & 8.7 & 7.3 & 7.0 \\
\hline $\mathrm{N}$ & 1.7 & 4.5 & 3.8 & 2.7 & 2.6 & 2.6 & 2.8 & 2.6 & 2.6 & 2.4 & 4.5 & 2.6 & 2.4 & 2.6 & 1.9 & 6.3 & 2.1 & 3.3 & 2.6 \\
\hline $\mathrm{K}$ & 2.1 & 7.3 & 5.3 & 3.7 & 3.0 & 2.7 & 3.1 & 3.1 & 2.9 & 3.3 & 7.0 & 2.7 & 2.8 & 2.7 & 2.3 & 9.2 & 2.3 & 3.6 & 3.0 \\
\hline $\mathrm{T}$ & 6.2 & 5.5 & 5.5 & 5.3 & 5.1 & 5.2 & 6.2 & 5.2 & 5.3 & 5.4 & 5.7 & 5.2 & 5.3 & 4.9 & 6.0 & 5.0 & 6.3 & 6.0 & 5.2 \\
\hline I & 3.0 & 7.9 & 6.6 & 5.4 & 5.2 & 4.1 & 5.5 & 5.1 & 4.8 & 4.3 & 7.4 & 4.3 & 4.4 & 4.0 & 3.5 & 9.8 & 4.2 & 4.9 & 5.0 \\
\hline $\mathrm{Q}$ & 2.7 & 3.8 & 3.9 & 3.0 & 3.3 & 4.5 & 3.4 & 3.0 & 3.2 & 3.1 & 4.2 & 3.2 & 3.2 & 4.4 & 2.7 & 2.5 & 3.2 & 3.8 & 3.2 \\
\hline $\mathrm{R}$ & 8.3 & 3.9 & 5.1 & 6.9 & 7.5 & 7.6 & 8.6 & 7.3 & 7.2 & 7.2 & 4.2 & 8.0 & 7.6 & 7.5 & 7.8 & 3.6 & 7.5 & 6.6 & 7.1 \\
\hline $\mathrm{H}$ & 2.3 & 2.2 & 2.1 & 2.0 & 2.1 & 2.3 & 2.2 & 2.0 & 2.1 & 1.8 & 2.0 & 2.4 & 1.8 & 2.2 & 2.2 & 1.4 & 0.4 & 2.2 & 1.9 \\
\hline$F$ & 2.7 & 4.6 & 4.1 & 3.9 & 3.5 & 3.2 & 3.9 & 3.6 & 3.5 & 3.5 & 4.7 & 3.6 & 3.4 & 3.3 & 3.0 & 4.4 & 2.7 & 3.9 & 3.7 \\
\hline$S$ & 5.1 & 6.0 & 6.4 & 5.7 & 5.4 & 5.6 & 5.7 & 5.2 & 5.4 & 5.2 & 5.0 & 5.4 & 5.1 & 5.4 & 5.2 & 6.4 & 6.4 & 6.3 & 5.4 \\
\hline $\mathrm{L}$ & 10.3 & 9.6 & 10.1 & 9.9 & 9.9 & 10.7 & 5.3 & 9.7 & 9.9 & 10 & 10.4 & 9.9 & 9.9 & 11.1 & 10.5 & 9.3 & 10.1 & 10 & 9.7 \\
\hline $\mathrm{Y}$ & 2.0 & 3.7 & 3.5 & 2.2 & 2.3 & 2.4 & 2.5 & 2.2 & 2.2 & 2.2 & 3.9 & 2.4 & 2.0 & 2.3 & 2.0 & 4.2 & 2.1 & 2.7 & 2.2 \\
\hline $\mathrm{W}$ & 1.5 & 1.0 & 1.3 & 1.4 & 1.5 & 1.6 & 1.6 & 1.5 & 1.5 & 1.5 & 0.9 & 1.4 & 1.5 & 1.6 & 1.5 & 0.8 & 1.5 & 1.4 & 1.4 \\
\hline $\mathrm{C}$ & 0.8 & 0.8 & 0.8 & 0.9 & 0.8 & 1.0 & 0.8 & 0.8 & 0.9 & 0.7 & 0.6 & 1.0 & 0.7 & 0.8 & 0.8 & 1.3 & 0.8 & 0.9 & 0.8 \\
\hline $\mathrm{P}$ & 6.3 & 3.5 & 4.1 & 5.1 & 5.3 & 5.3 & 6.1 & 5.3 & 5.4 & 5.6 & 3.3 & 5.4 & 5.5 & 5.3 & 6.0 & 2.9 & 5.8 & 5.4 & 5.1 \\
\hline Weight of average & 16.7 & 10 & 5.5 & 3.6 & 3.5 & 3.0 & 2.8 & 2.4 & 2.4 & 2.0 & 1.5 & 1.3 & 1.2 & 1.1 & 1.1 & 1.0 & 0.9 & 0.6 & 0.6 \\
\hline
\end{tabular}


Table S9. Oligonucleotide primers used in this study. All sequences are provided in the $5^{\prime}$ to $3^{\prime}$ direction. "f" and " $r$ " indicate a forward or reverse primer, respectively. LP, leader peptide; CP, core peptide; capital letters, mutagenized codon; capitalized and italics, restriction enzyme recognition site.

\begin{tabular}{|c|c|c|}
\hline Number & Primer name & Oligonucleotide sequence \\
\hline 1 & Cellulassin BGC_f & aaaGGATCCatgcaggaaaagaagaccC \\
\hline 2 & Cellulassin BGC_r & aaaGCGGCCGCtcagtcgacggtgatcagg \\
\hline 3 & FusA LP $_{\text {-chimeric_f }}$ & cgcGGATCCatggaaaagaagaagtacac \\
\hline 4 & Fus $_{\mathrm{LP}}-\mathrm{CelA} \mathrm{CP}_{\mathrm{CP}} \mathrm{r}$ & CCCAAGCT TCtacaatagcg cgggaag \\
\hline 5 & Fus $_{L P}-\mathrm{HalA}_{\mathrm{CP} \_} \mathrm{r}$ & cCCAAGCTTttataccatacgggggag \\
\hline 6 & FusA $_{\mathrm{LP}}-\mathrm{Mth} \mathrm{A}_{\mathrm{CP} \_} \mathrm{r}$ & CCCAAGCTTttaattgaataaacgaggccag \\
\hline 7 & FusA $A_{L P}-N_{c a A} A_{C P} r$ & cCCAAGCTTttaccagatattacggggg \\
\hline 8 & FusA $_{\mathrm{LP}}-\mathrm{Mob} \mathrm{A}_{\mathrm{CP} \_} \mathrm{r}$ & cCCAAGCTTttaccagaacttggagaacg \\
\hline 9 & FusA $_{\mathrm{LP}}-\mathrm{Nbs} \mathrm{A}_{\mathrm{CP} \_} \mathrm{r}$ & cCCAAGCTTttacccaccgaaagtaccg \\
\hline 10 & FusA $_{\mathrm{LP}}-\mathrm{Sle} \mathrm{A}_{\mathrm{CP} \_} \mathrm{r}$ & CCCAAGCTTttaagtccagtagtcgaagtg \\
\hline 11 & FusA $_{L P}-R u b A_{C P} \_r$ & CCCAAGCTTttaccaccacgacgtgtg \\
\hline 12 & FusA $\mathrm{AP}_{\mathrm{LP}}-\mathrm{SruA} \mathrm{A}_{\mathrm{CP} \_} \mathrm{r}$ & cCCAAGCTTttaaacccagtagtcccatg \\
\hline 13 & FusA $A_{L_{P}}-A_{r e} A_{C P}{ }_{C}$ & cCCAAGCTTtcaggccttcgtttgg \\
\hline 14 & FusA_Y2A_f & ccaccggctggGCGaccgcggaatggggcctcgagc \\
\hline 15 & FusA_Y2A_r & cattccgcggtCGCccagccggtggcctccttgaat \\
\hline 16 & FusA_T3A_f & ccggctggtacGCCgcggaatggggcctcgagctga \\
\hline 17 & FusA_T3A_r & ccccattccgcCCGgtaccagccggtggcctccttg \\
\hline 18 & FusA_E5A_f & ggtacaccgcgGCCtggggcctcgagctgatcttcg \\
\hline 19 & FusA_E5A_r & tcgaggccccaGGCcgcggtg taccagccggtggec \\
\hline 20 & FusA_W6A_f & acaccgcggaaGCGggcctcgagctgatcttcgtct \\
\hline 21 & FusA_W6A_r & agctcgaggccCGCttccgcggtgtaccagccggtg \\
\hline 22 & FusA_G7A_f & ccgcggaatggGCGctcgagctgatcttcgtcttcc \\
\hline 23 & FusA_G7A_r & atcagctcgagCGCccattccgcggtgtaccagccg \\
\hline 24 & FusA_L8A_f & cggaatggggcGCGgagctgatcttcgtcttcccgc \\
\hline 25 & FusA_L8A_r & a agatcagctcCGCgccccattccgcggtgtaccag \\
\hline 26 & FusA_L10A_f & ggggcctcgagGCGatcttcgtcttcccgcgcttca \\
\hline 27 & FusA_L10A_r & aagacgaagatCGCctcgagg ccccattccgcggtg \\
\hline 28 & FusA_I11A_f & gcctcgagctgGCGttcgtcttcccgcgcttcatct \\
\hline 29 & FusA_I11A_r & gggaagacgaaCGCcagctcgaggccccattccgcg \\
\hline 30 & FusA_F12A_f & tcgagctgatcGCGgtcttcccgcgcttcatctgag \\
\hline 31 & FusA_F12A_r & cgcgggaagacCGCgatcagctcgaggccccattcc \\
\hline 32 & FusA_V13A_f & agctgatcttcGCGttcccgcgcttcatctgagcgg \\
\hline 33 & FusA_V13A_r & a agcgcgggaaCGCgaagatcagctcgaggccccat \\
\hline
\end{tabular}




\begin{tabular}{|c|c|c|}
\hline 34 & FusA_F14A_f & tgatcttcgtcGCGccgcgcttcatctgagcggccg \\
\hline 35 & FusA_F14A_r & atgaagcgcggCGCgacgaagatcagctcgaggccC \\
\hline 36 & FusA_P15A_f & tcttcgtcttcGCGcgcttcatctgagcggccgcac \\
\hline 37 & FusA_P15A_r & cagatgaagcgCGCgaagacgaagatcagctcgag \\
\hline 38 & FusA_R16A_f & tcgtcttcccgGCGttcatctgagcggccgcactc \\
\hline 39 & FusA_R16A_r & gctcagatgaaCGCcgggaagacgaagatcagctc \\
\hline 40 & FusA_F17A_f & tcttcccgcgcGCGatctgagcggccgcactcgag \\
\hline 41 & FusA_F17A_r & gccgctcagatCGCgcgcgggaagacgaagatcag \\
\hline 42 & FusA_I18A_f & cttcccgcgcttcGCGtgagcggccgcactcgagc \\
\hline 43 & FusA_I18A_r & cggccgctcaCGCgaagcgcgggaagacgaagatc \\
\hline 44 & pET28_CFB_linear DNA_f & gctatcatgccataccgcgaaaggttttgcgccattcg \\
\hline 45 & pET28_CFB_linear DNA_r & aaccgtctatcagggcgatggcccactacgtgaaccatc \\
\hline 46 & FusA DEGlib_KpnI_f & cgcGGTACCatcccgcgaaattaatacgactcactatagg \\
\hline 47 & FusA DEGlib_SacI_r & cgcGAGCTCcaaaaaacccctcaagacccgtttagagg \\
\hline 48 & pET28_DEGlib_SacI_f & cgCGAGCTCCtgaaaggaggaactatatcC \\
\hline 49 & pET28_DEGlib_KpnI_r & cggGGTACCcgagatctcgatcctctac \\
\hline 50 & MthA_ring to FusA_f & TACACCGCGGAATGGGGCttagagatcatcttcatctgg \\
\hline 51 & NcaA_ring to FusA_f & TACACCGCGGAATGGGGCcgtgagtcgcttttagggtac \\
\hline 52 & MobA_ring to FusA_f & TACACCGCGGAATGGGGCagcgagcctatcactcactcg \\
\hline 53 & NbsA_ring to FusA_f & TACACCGCGGAATGGGGCtacgagaatgtaatccacttc \\
\hline 54 & $\begin{array}{l}\text { MthA_CanA_MobA_NbsA_ring } \\
\text { to FusA_r }\end{array}$ & GCCCCATTCCGCGGTGTAgtaacctgttgcttccttaaac \\
\hline 55 & MthA_loop to FusA_f & CTGATCTTCGTCTTCCCGcgtttattcaattaaaagcttgcg \\
\hline 56 & NcaA_loop to FusA_f & CTGATCTTCGTCTTCCCGcgtaatatctggtaaaagcttgcg \\
\hline 57 & MobA_loop to FusA_f & CTGATCTTCGTCTTCCCGaagttctggtaaaagcttgcg \\
\hline 58 & NbsA_loop to FusA_f & CTGATCTTCGTCTTCCCGcgtctctaaaagcttgcg \\
\hline 59 & MthA_loop to FusA_r & CGGGAAGACGAAGATCAGctctaacttattgatggcattg \\
\hline 60 & NcaA_loop to FusA_r & CGGGAAGACGAAGATCAGctcacgattacgcaatccgac \\
\hline 61 & MobA_loop to FusA_r & CGGGAAGACGAAGATCAGctcgcttccetcgagacc \\
\hline 62 & NbsA_loop to FusA_r & CGGGAAGACGAAGATCAGctcgtacccagtcaaacc \\
\hline 63 & FusA ring_MNMYM_f & ATGAACATGTATATGggcctcgagctgatcttcgtcttcccg \\
\hline 64 & FusA ring_MNMYM_r & CATATACATGTTCATCcagccggtggcetccttgaattcg \\
\hline 65 & FusA ring_FYNWK_f & TTTTATAACTGGAAAggcctcgagctgatcttcgtcttcccg \\
\hline 66 & FusA ring_FYNWK_r & TTTCCAGTTATAAAACCagccggtggcctccttgaattcg \\
\hline 67 & FusA ring_NMQIY_f & AACATGCAGATTTATggcctcgagctgatcttcgtcttcccg \\
\hline 68 & FusA ring_NMQIY_r & ATAAATCTGCATGTTCcagccggtggcetccttgaattcg \\
\hline 69 & FusA ring_MYTFQ_f & ATGTATACCTTTCAGggcctcgagctgatcttcgtcttcccg \\
\hline 70 & FusA ring_MYTFQ_r & CTGAAAGGTATACATccagccggtggcetccttgaattcg \\
\hline
\end{tabular}




\begin{tabular}{|c|c|c|}
\hline 71 & FusA ring_TWTEM_f & ACCTGGACCGAAATGggcctcgagctgatcttcgtcttcccg \\
\hline 72 & FusA ring_TWTEM_r & CATTTCGGTCCAGGTccagccggtggcctccttgaattcg \\
\hline 73 & FusA ring_IKEVT_f & ATTAAAGAAGTGACCggcctcgagctgatcttcgtcttcccg \\
\hline 74 & FusA ring_IKEVT_r & GGTCACTTCTTTAATCCagccggtggcctccttgaattcg \\
\hline 75 & FusA ring_QDWFM_f & CAGGATTGGTTTATGggcctcgagctgatcttcgtcttcccg \\
\hline 76 & FusA ring_QDWFM_r & CATAAACCAATCCTGCCagccggtggcetccttgaattcg \\
\hline 77 & FusA ring_FLRCL_f & TTTCTGCGCTGCCTGggcetcgagctgatcttcgtcttcccg \\
\hline 78 & FusA ring_FLRCL_r & CAGGCAGCGCAGAAAccagccggtggcctccttgaattcg \\
\hline 79 & FusA ring_IDRSY_f & ATTGATCGCAGCTATggcctcgagctgatcttcgtcttcccg \\
\hline 80 & FusA ring_IDRSY_r & ATAGCTGCGATCAATccagccggtggcctccttgaattcg \\
\hline 81 & FusA ring_LKNFT_f & CTGAAAAACTTTACCggcctcgagctgatcttcgtcttcccg \\
\hline 82 & FusA ring_LKNFT_r & GGTAAAGTTTTTCAGccagccggtggcctccttgaattcg \\
\hline
\end{tabular}


A

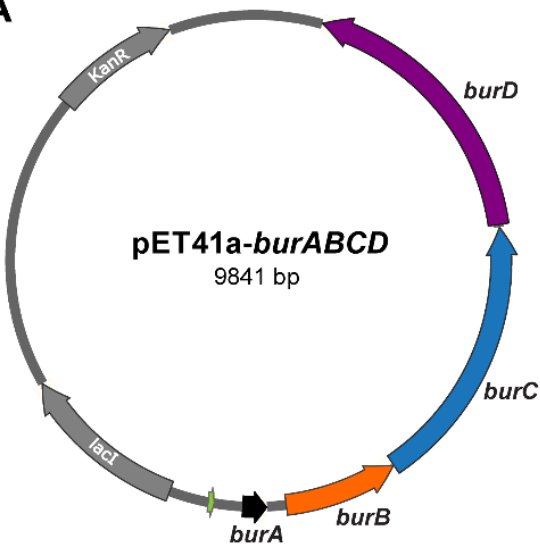

B

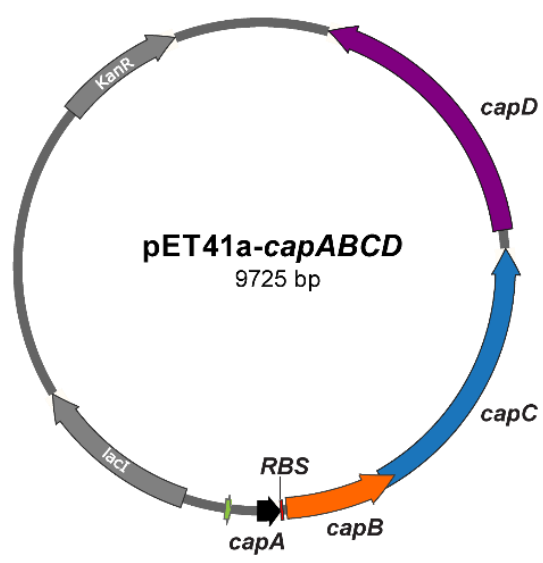

C

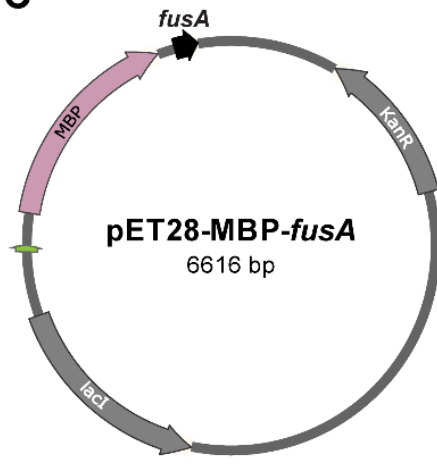

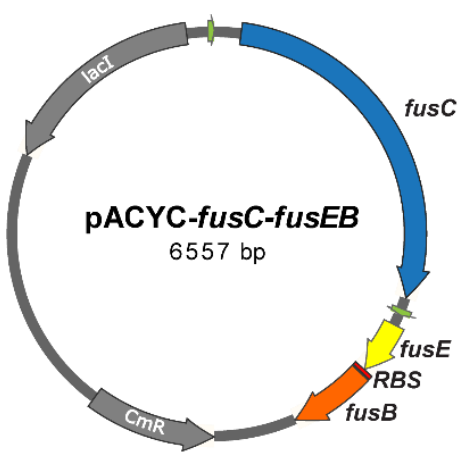

D

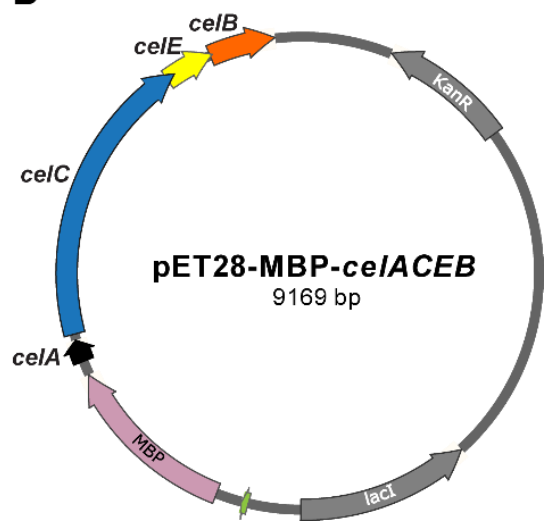

Figure S1. Plasmid maps for constructs used in CFB reactions. (A) Plasmid map of pET41a-burABCD. The BGC of burhizin from Burkholderia rhizoxinica was cloned into pET41a using the native gene organization. (B) Plasmid map of pET41a-capABCD. The BGC of capistruin from Burkholderia thailandensis was cloned into pET41a using the native organization with the intergenic region between capA and $c a p B$ replaced with an E. coli ribosome-binding site (RBS). (C) Plasmid maps of pET28-MBP-fusA and pACYC-fusC-fusEB. The fusA (fusilassin precursor peptide) gene (or chimeric precursor) was cloned into pET28-MBP. The lasso cyclase gene (fusC) was cloned into the first multiple cloning site of pACYC. The RiPP recognition element (fusE) and leader peptidase ( $f u s B$ ) were Gibson assembled into the second multiple cloning site of pACYC with an $E$. coli RBS inserted between fusE and fusB. ${ }^{2}$ (D) The plasmid map of pET28-MBP-celACEB. The BGC of cellulassin from Thermobifida cellulosilytica was cloned into pET28-MBP using the native organization. Green, T7 promotor; Red, inserted RBS. The NCBI accession identifiers for the cellulassin biosynthetic proteins are: CelA, WP_157080207.1; CelC, WP_068757176.1; CelE, WP_068757174.1; CelB, WP_068757172.1. 
A

Formula: $\mathrm{C}_{84} \mathrm{H}_{129} \mathrm{~N}_{27} \mathrm{O}_{27}$

Cal. $[\mathrm{M}+2 \mathrm{H}]^{2+}: 924.4428 \mathrm{Da}$

Obsv. $[\mathrm{M}+2 \mathrm{H}]^{2+}:$ 924.4417 Da

Error: $1.19 \mathrm{ppm}$

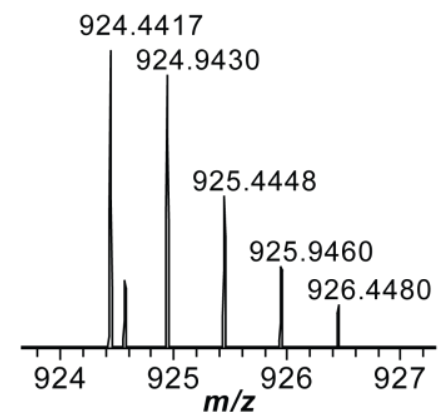

C

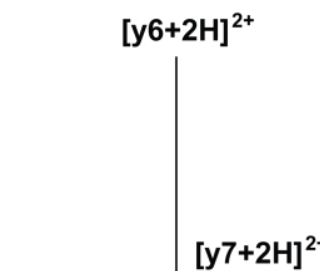

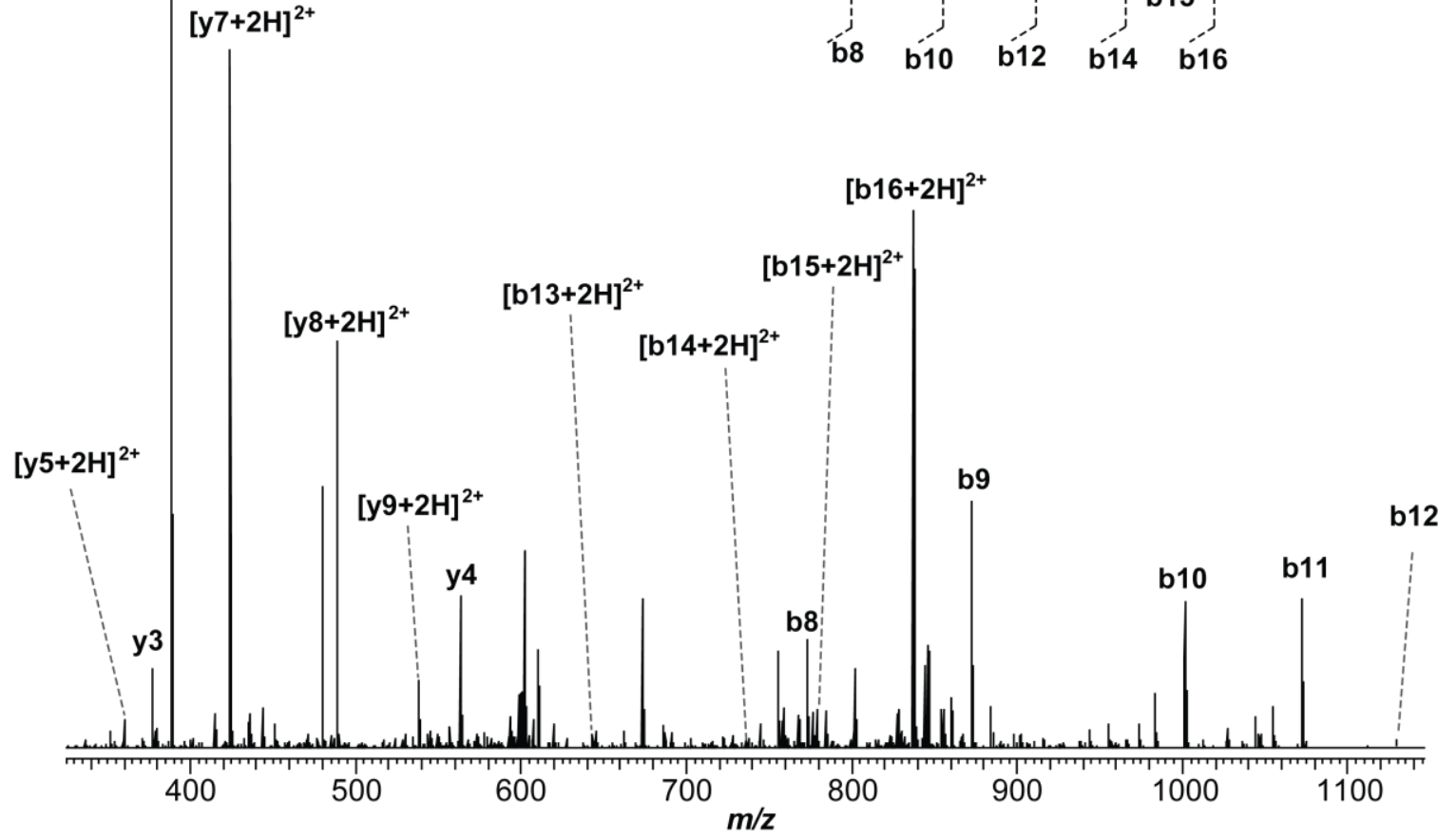

B

\begin{tabular}{|c|c|c|c|}
\hline Ion & $\begin{array}{c}\text { Calculated } \\
\text { mass (Da) }\end{array}$ & $\begin{array}{c}\text { Observed } \\
\text { mass (Da) }\end{array}$ & $\begin{array}{c}\text { Error } \\
\text { (ppm) }\end{array}$ \\
\hline $\mathrm{b} 8$ & 773.3582 & 773.3552 & 3.88 \\
\hline $\mathrm{b} 9$ & 872.4266 & 872.4242 & 2.75 \\
\hline $\mathrm{b} 10$ & 1001.4692 & 1001.4668 & 2.40 \\
\hline $\mathrm{b} 11$ & 1072.5063 & 1072.5035 & 2.61 \\
\hline $\mathrm{b} 12$ & 1129.5278 & 1129.5265 & 1.15 \\
\hline$[\mathrm{b} 13+2 \mathrm{H}]^{2+}$ & 643.3178 & 643.3170 & 1.24 \\
\hline$[\mathrm{b} 14+2 \mathrm{H}]^{2+}$ & 736.3575 & 736.3556 & 2.58 \\
\hline$[\mathrm{b} 15+2 \mathrm{H}]^{2+}$ & 779.8734 & 779.8697 & 4.74 \\
\hline$[\mathrm{b} 16+2 \mathrm{H}]^{2+}$ & 837.3868 & 837.3849 & 2.27 \\
\hline $\mathrm{y} 3$ & 377.1785 & 377.1767 & 4.77 \\
\hline $\mathrm{y} 4$ & 563.2578 & 563.2563 & 2.66 \\
\hline$[\mathrm{y} 5+2 \mathrm{H}]^{2+}$ & 360.1828 & 360.1816 & 3.33 \\
\hline$[\mathrm{y} 6+2 \mathrm{H}]^{2+}$ & 388.6925 & 388.6924 & 0.26 \\
\hline$[\mathrm{y} 7+2 \mathrm{H}]^{2+}$ & 424.2121 & 424.2110 & 2.59 \\
\hline$[\mathrm{y} 8+2 \mathrm{H}]^{2+}$ & 488.7334 & 488.7325 & 1.84 \\
\hline$[\mathrm{y} 9+2 \mathrm{H}]^{2+}$ & 538.2676 & 538.2668 & 1.49 \\
\hline
\end{tabular}<smiles>[GeH2]O[GeH2]</smiles>

G) $\mathrm{O}^{-\mathrm{NH}} \mathrm{y}^{\mathrm{y}} \quad \mathrm{y}^{6} \quad \mathrm{y}^{4}$ Q $y 9$ y7 $y 5$ y3

(R) (V) A G R WOSDR- $\mathrm{CO}_{2} \mathrm{H}$

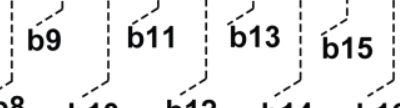

$[\mathrm{b} 16+2 \mathrm{H}]^{2+}$

Figure S2. High-resolution and tandem MS of burhizin produced from CFB. (A) High-resolution mass spectrum of burhizin, produced from CFB with pET41a-burABCD as the DNA template. (B) Mass assignment for the $\mathrm{b}^{+}$and $\mathrm{y}^{+}$ions generated from CID of burhizin. (C) Tandem MS spectrum of burhizin, consistent with a Gly1-Glu8 macrolactam. 
A

Formula: $\mathrm{C}_{98} \mathrm{H}_{148} \mathrm{~N}_{28} \mathrm{O}_{27}$

Cal. $[\mathrm{M}+2 \mathrm{H}]^{2+}: 1025.0187 \mathrm{Da}$

Obsv. $[\mathrm{M}+2 \mathrm{H}]^{2+}: 1025.0177 \mathrm{Da}$

Error: $0.98 \mathrm{ppm}$

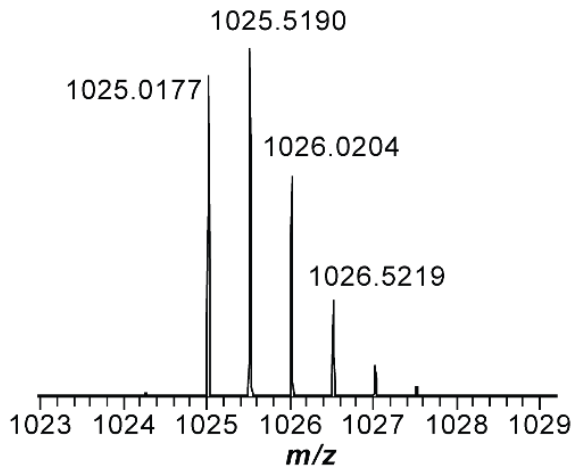

C

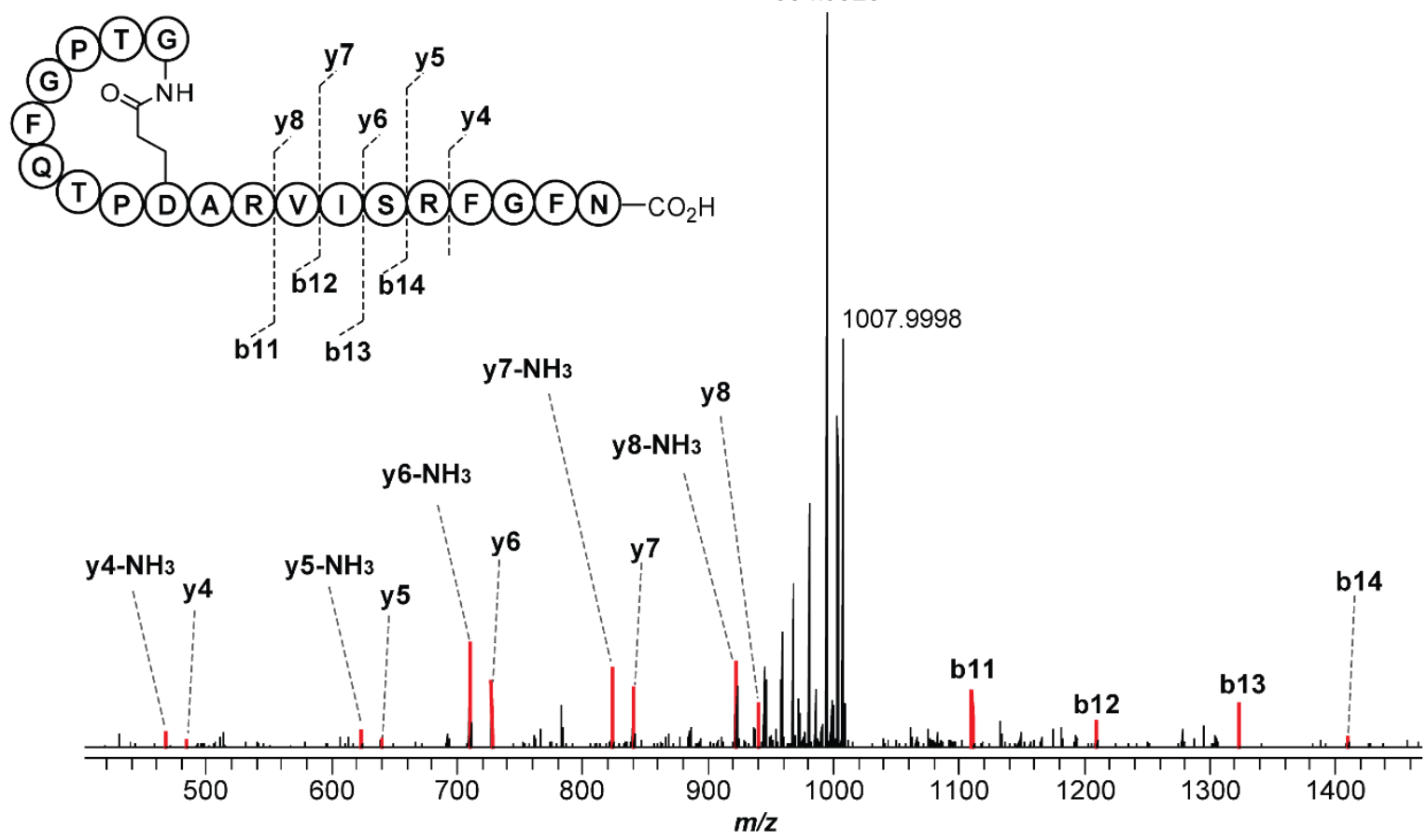

B

\begin{tabular}{|c|c|c|c|}
\hline Ion & $\begin{array}{c}\text { Calculated } \\
\text { mass (Da) }\end{array}$ & $\begin{array}{c}\text { Observed } \\
\text { mass (Da) }\end{array}$ & Error (ppm) \\
\hline $\mathrm{b} 11$ & 1110.5332 & 1110.5311 & 1.89 \\
\hline $\mathrm{b} 12$ & 1209.6016 & 1209.5990 & 2.15 \\
\hline $\mathrm{b} 13$ & 1322.6857 & 1322.6831 & 1.97 \\
\hline $\mathrm{b} 14$ & 1409.7177 & 1409.7146 & 2.20 \\
\hline $\mathrm{y} 4-\mathrm{NH}_{3}$ & 467.1931 & 467.1922 & 1.93 \\
\hline $\mathrm{y} 4$ & 484.2196 & 484.2187 & 1.86 \\
\hline $\mathrm{y} 5-\mathrm{NH}_{3}$ & 623.2942 & 623.2927 & 2.41 \\
\hline $\mathrm{y} 5$ & 640.3207 & 640.3195 & 1.87 \\
\hline $\mathrm{y} 6-\mathrm{NH}_{3}$ & 710.3262 & 710.3249 & 1.83 \\
\hline $\mathrm{y} 6$ & 727.3527 & 727.3513 & 1.92 \\
\hline $\mathrm{y} 7-\mathrm{NH}_{3}$ & 823.4103 & 823.4088 & 1.82 \\
\hline $\mathrm{y} 7$ & 840.4368 & 840.4353 & 1.78 \\
\hline $\mathrm{y} 8-\mathrm{NH}_{3}$ & 922.4787 & 922.4769 & 1.95 \\
\hline $\mathrm{y} 8$ & 939.5052 & 939.5036 & 1.70 \\
\hline & & &
\end{tabular}

994.9926

Figure S3. High-resolution and tandem MS of capistruin produced from CFB. (A)

High-resolution mass spectrum of capistruin produced from CFB with pET41a-capABCD as the DNA template. (B) Mass assignment for the $\mathrm{b}^{+}$and $\mathrm{y}^{+}$ions generated from CID of capistruin. (C) Tandem MS spectrum of capistruin. The most intense signals $(\mathrm{m} / \mathrm{z} 994.9926$ and 1007.9998) correspond to the neutral loss of multiple molecules of water and/or ammonia, consistent with previous reports on capistruin. ${ }^{4,18}$ Assigned $\mathrm{b}$ and $\mathrm{y}$ ions are red. 
A

Formula: $\mathrm{C}_{117} \mathrm{H}_{157} \mathrm{~N}_{23} \mathrm{O}_{24}$

Cal. $[\mathrm{M}+2 \mathrm{H}]^{2+}: 1135.0959 \mathrm{Da}$

Obsv. $[\mathrm{M}+2 \mathrm{H}]^{2+}: 1135.0952 \mathrm{Da}$

Error: $0.62 \mathrm{ppm}$

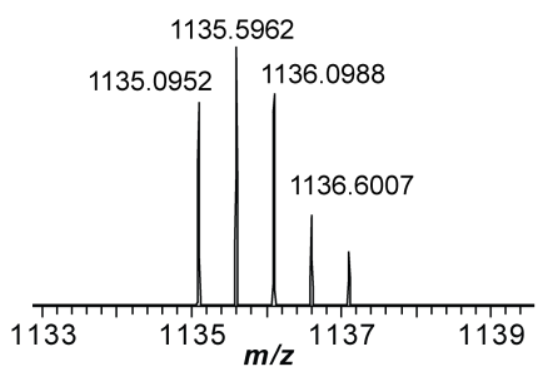

B

\begin{tabular}{|c|c|c|c|}
\hline Ion & $\begin{array}{c}\text { Calculated } \\
\text { mass (Da) }\end{array}$ & $\begin{array}{c}\text { Observed } \\
\text { mass (Da) }\end{array}$ & Error (ppm) \\
\hline b9 & 1118.4947 & 1118.4922 & 2.24 \\
\hline b10 & 1231.5788 & 1231.5760 & 2.27 \\
\hline b11 & 1344.6628 & 1344.6600 & 2.08 \\
\hline b12 & 1491.7313 & 1491.7286 & 1.81 \\
\hline b13 & 1590.7997 & 1590.7969 & 1.76 \\
\hline b14 & 1737.8681 & 1737.8643 & 2.19 \\
\hline y4 & 532.3247 & 532.3234 & 2.44 \\
\hline y5 & 679.3932 & 679.3917 & 2.21 \\
\hline y6 & 778.4616 & 778.4600 & 2.06 \\
\hline y7 & 925.5300 & 925.5283 & 1.84 \\
\hline y8 & 1038.6140 & 1038.6118 & 2.12 \\
\hline y9 & 1151.6981 & 1151.6952 & 2.52 \\
\hline
\end{tabular}

C
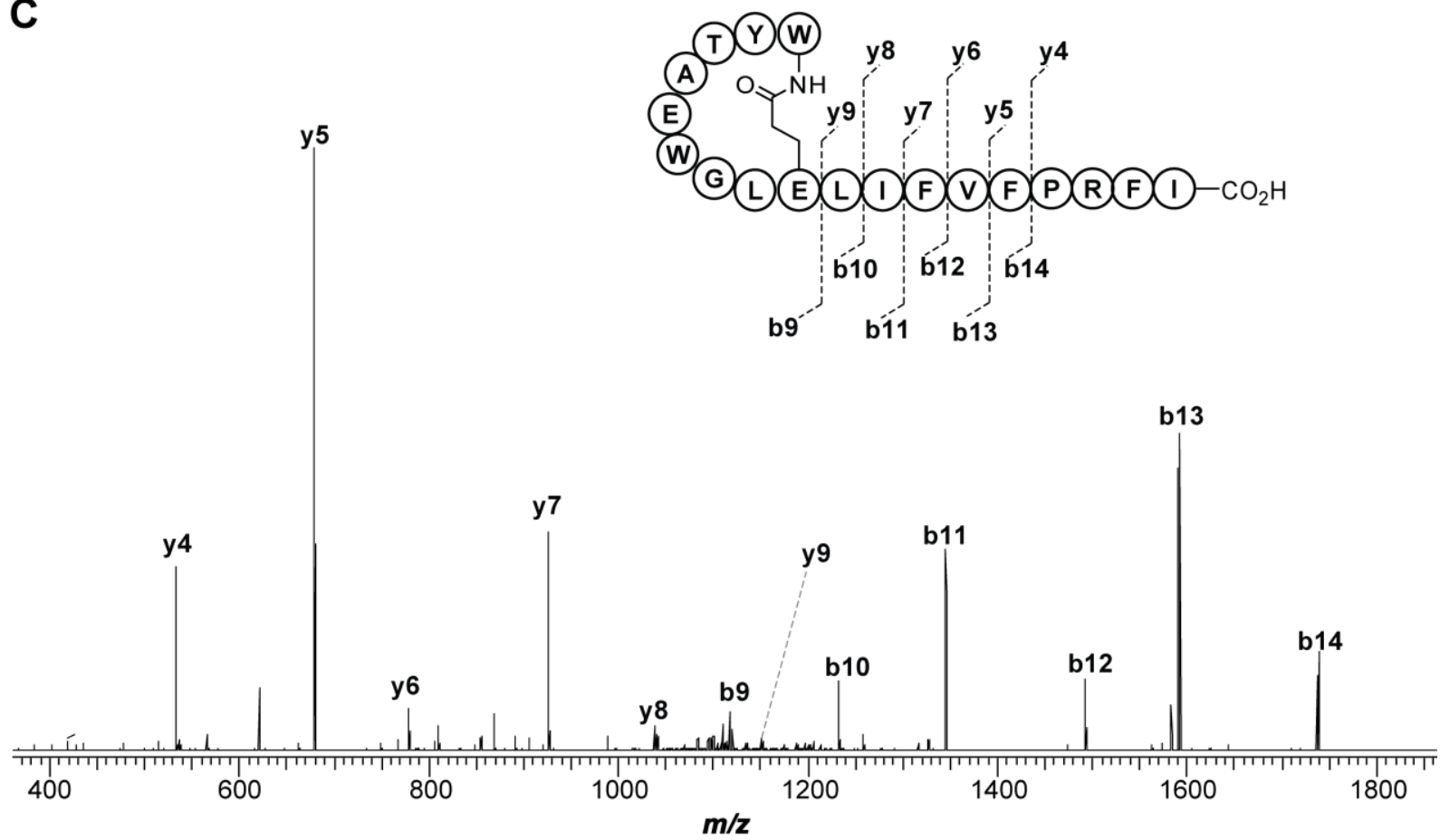

Figure S4. High-resolution and tandem MS of fusilassin produced from CFB. (A) High-resolution mass spectrum of fusilassin, produced from CFB with pET28-MBP-fusA and pACYC-fusC-fusEB as the DNA templates. (B) Mass assignment for the $\mathrm{b}^{+}$and $\mathrm{y}^{+}$ions generated the CID of fusilassin (C) Tandem MS spectrum of fusilassin, consistent with a Trp1-Glu9 macrolactam. 
A

Formula: $\mathrm{C}_{116} \mathrm{H}_{165} \mathrm{~N}_{25} \mathrm{O}_{23}$

Cal. $[\mathrm{M}+2 \mathrm{H}]^{2+}: 1139.1328 \mathrm{Da}$

Obsv. $[\mathrm{M}+2 \mathrm{H}]^{2+}: 1139.1318 \mathrm{Da}$

Error: $0.88 \mathrm{ppm}$

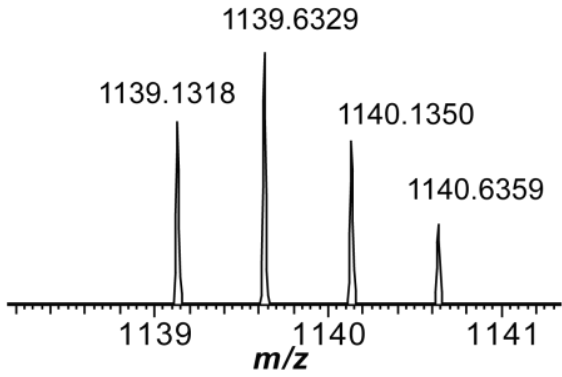

C

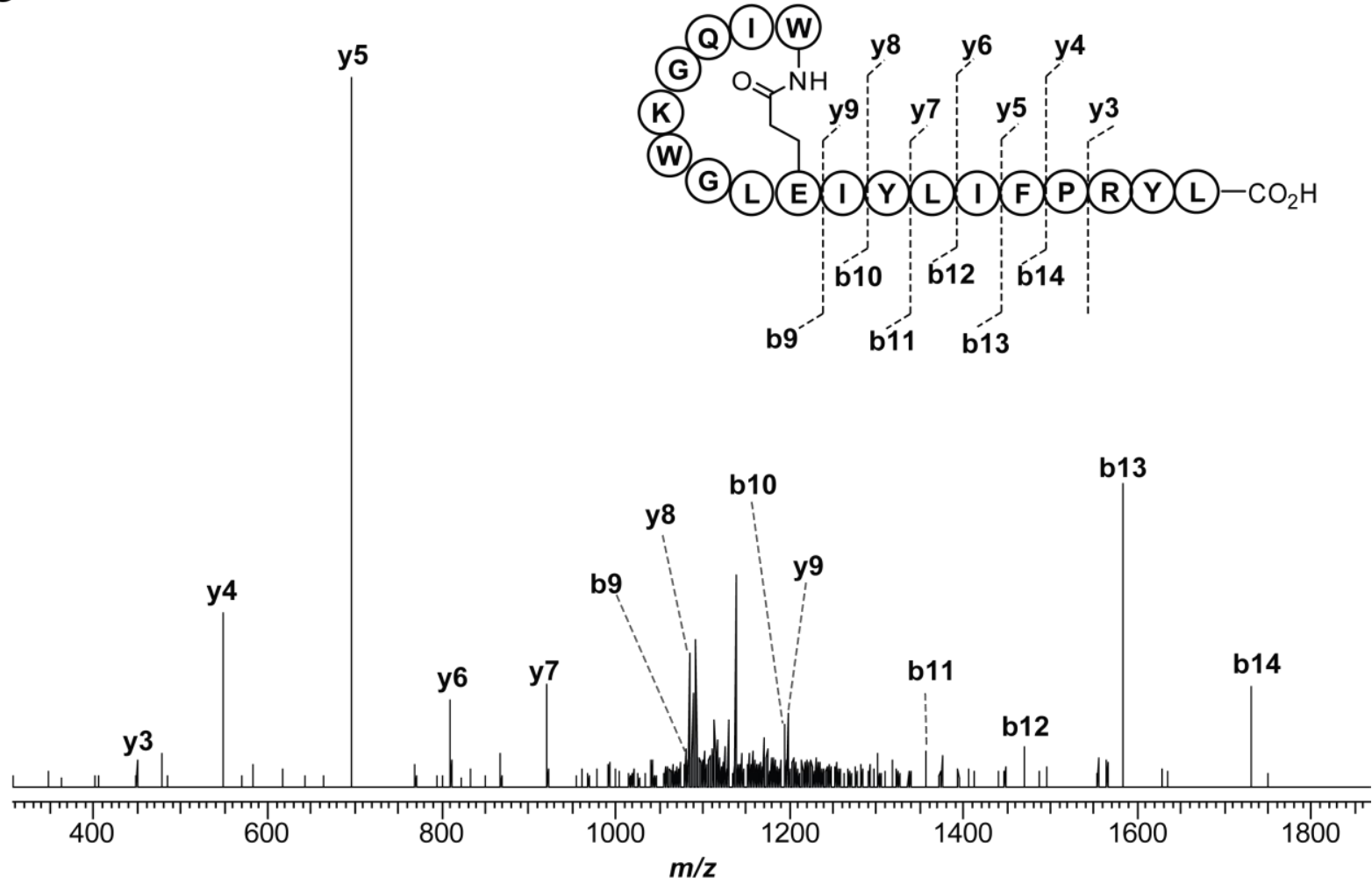

B

\begin{tabular}{|c|c|c|c|}
\hline Ion & $\begin{array}{c}\text { Calculated } \\
\text { mass (Da) }\end{array}$ & $\begin{array}{c}\text { Observed } \\
\text { mass (Da) }\end{array}$ & Error (ppm) \\
\hline b9 & 1080.5631 & 1080.5613 & 1.67 \\
\hline b10 & 1193.6471 & 1193.6454 & 1.42 \\
\hline b11 & 1356.7105 & 1356.7063 & 3.10 \\
\hline b12 & 1469.7945 & 1469.7930 & 1.02 \\
\hline b13 & 1582.8786 & 1582.8760 & 1.64 \\
\hline b14 & 1730.9470 & 1730.9463 & 0.40 \\
\hline y3 & 451.2669 & 451.2660 & 1.99 \\
\hline y4 & 548.3197 & 548.3182 & 2.74 \\
\hline y5 & 695.3881 & 695.3865 & 2.30 \\
\hline y6 & 808.4721 & 808.4708 & 1.61 \\
\hline y7 & 921.5562 & 921.5546 & 1.74 \\
\hline y8 & 1084.6195 & 1084.6152 & 3.96 \\
\hline y9 & 1197.7036 & 1197.7007 & 2.42 \\
\hline
\end{tabular}

Figure S5. High-resolution and tandem MS of cellulassin produced from CFB. (A) High-resolution broadband spectrum of cellulassin, produced from CFB with pET28-MBP-celACEB as the DNA template. (B) Mass assignment for the $\mathrm{b}^{+}$and $\mathrm{y}^{+}$ions generated from CID of cellulassin. (C) Tandem MS spectrum of cellulassin, consistent with a Trp1-Glu9 macrolactam. 


\section{Fusilassin}

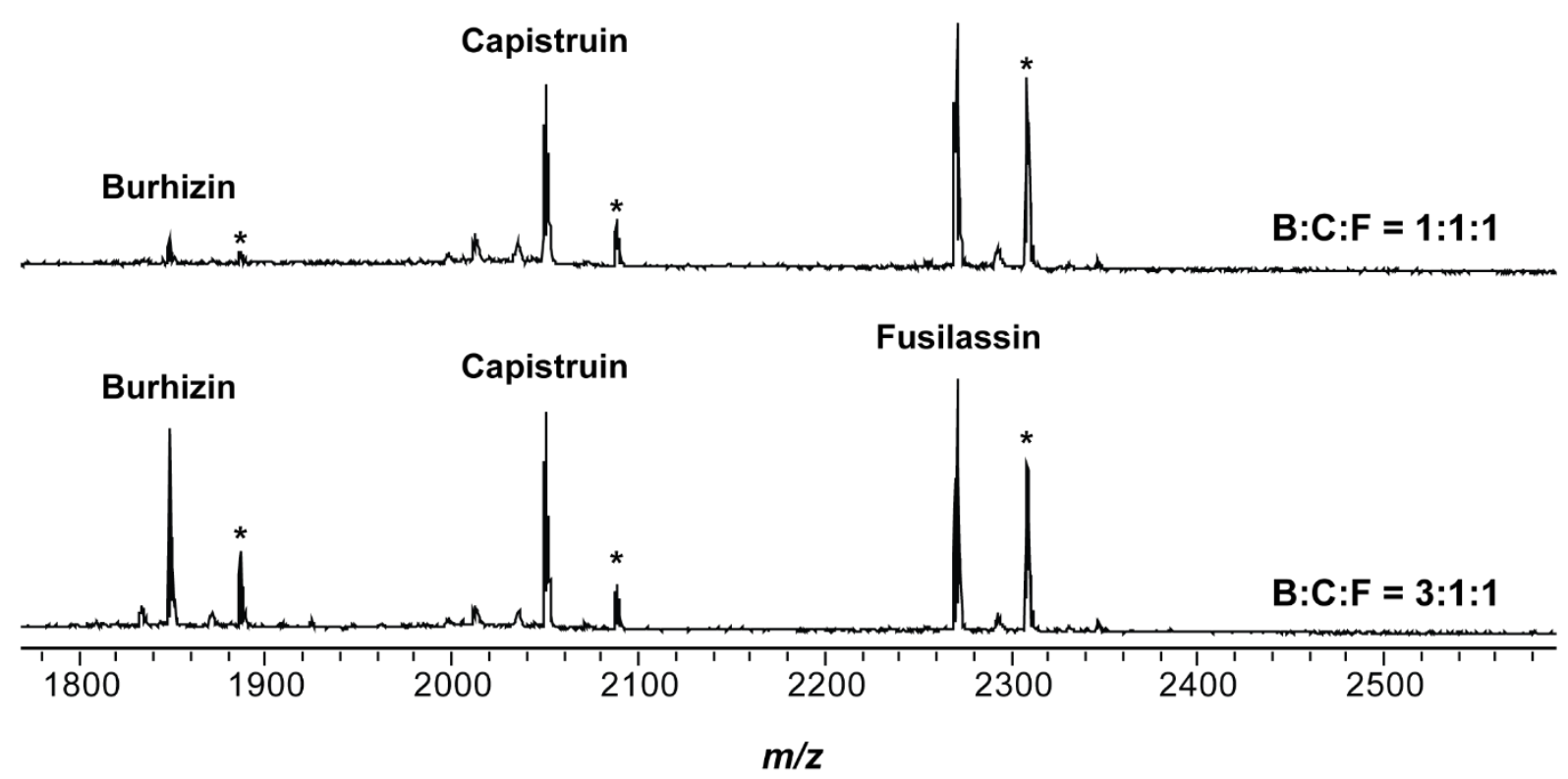

Figure S6. Simultaneous production of multiple lasso peptides using CFB. MALDI-TOF mass spectrum of burhizin, capistruin, and fusilassin produced in a single CFB reaction. Different ratios of DNA templates encoding the BGC of burhizin, capistruin, and fusilassin were supplied in a single CFB reaction. Top, the ratio of DNA templates is 1:1:1 for burhizin, capistruin, and fusilassin respectively; bottom, the ratio of DNA templates 3:1:1. B, burhizin; C, capistruin; F, fusilassin. * indicates $[\mathrm{M}+\mathrm{K}]^{+}$ion of the lasso peptide. 

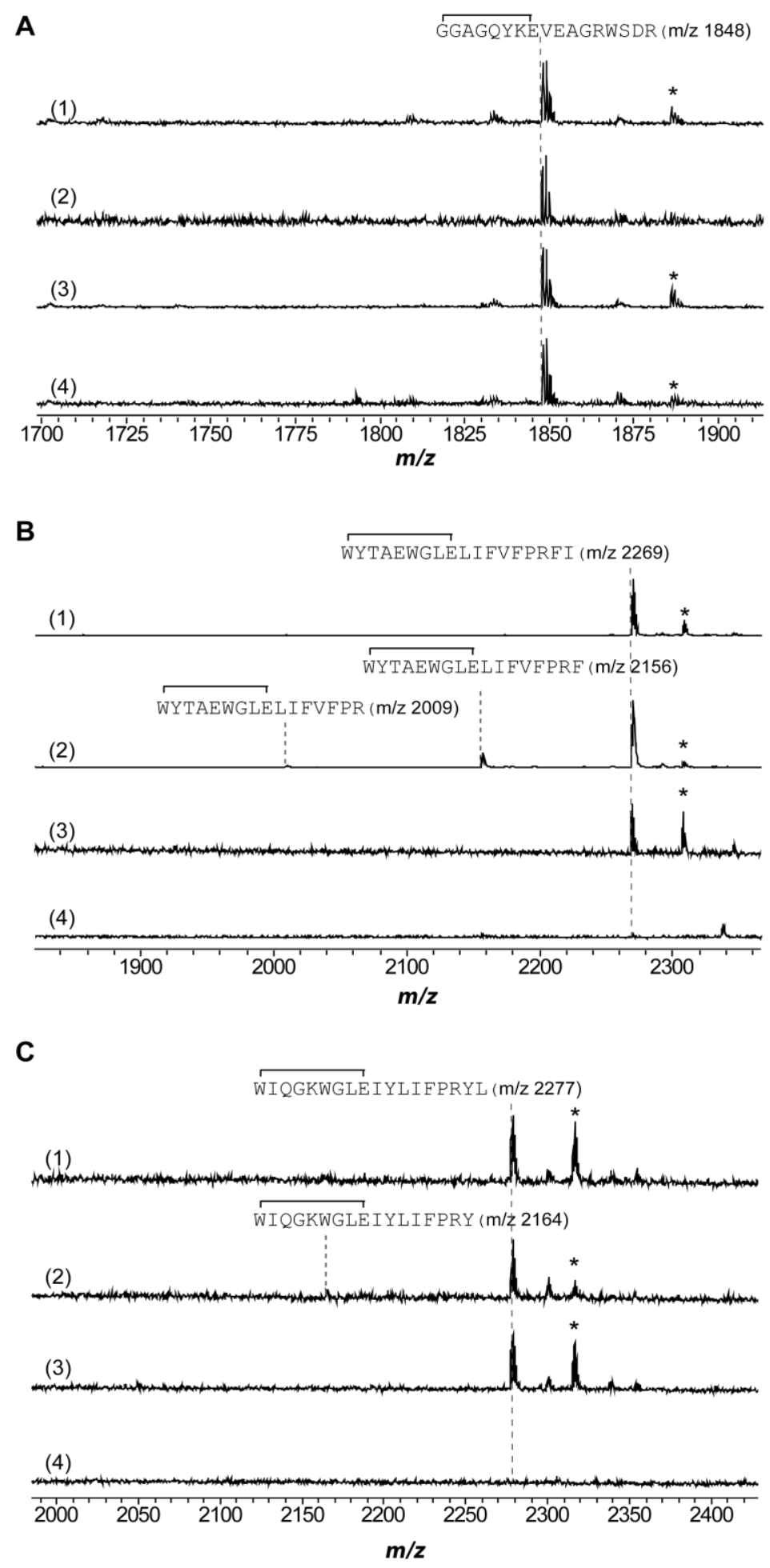

Figure S7. Carboxypeptidase $\mathrm{Y}$ resistance of CFB-produced lasso peptides. Burhizin (A), fusilassin (B), and cellulassin (C) were produced by CFB and shown in the top spectrum (1). Each lasso peptide was subjected to carboxypeptidase $\mathrm{Y}$ digestion for $18 \mathrm{~h}$ at room temperature (2); heat treatment at $95{ }^{\circ} \mathrm{C}$ for $2 \mathrm{~h}$ in $60 \%$ ACN (3); heat treatment at $95{ }^{\circ} \mathrm{C}$ for $2 \mathrm{~h}$ in $60 \%$ ACN followed by carboxypeptidase $\mathrm{Y}$ digestion for $18 \mathrm{~h}$ at room temperature (4). * indicates $[\mathrm{M}+\mathrm{K}]^{+}$ion of the lasso peptide. 


\section{A}
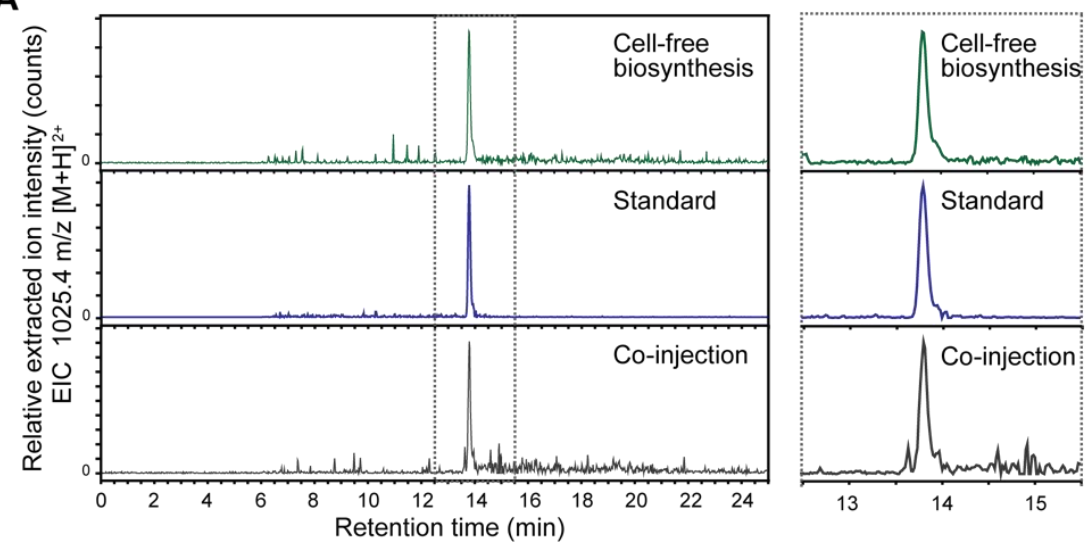

B

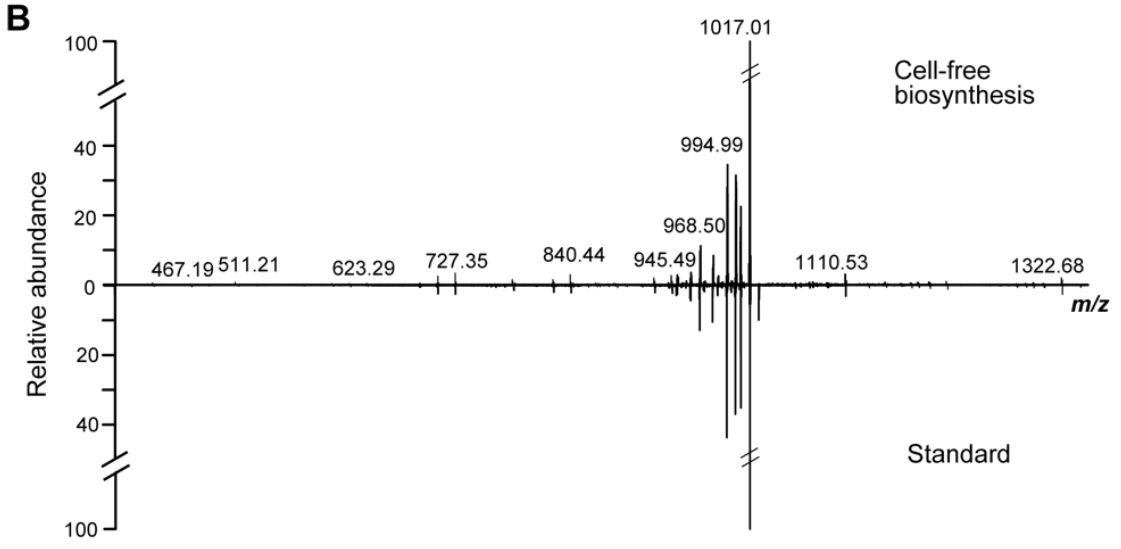

C

D
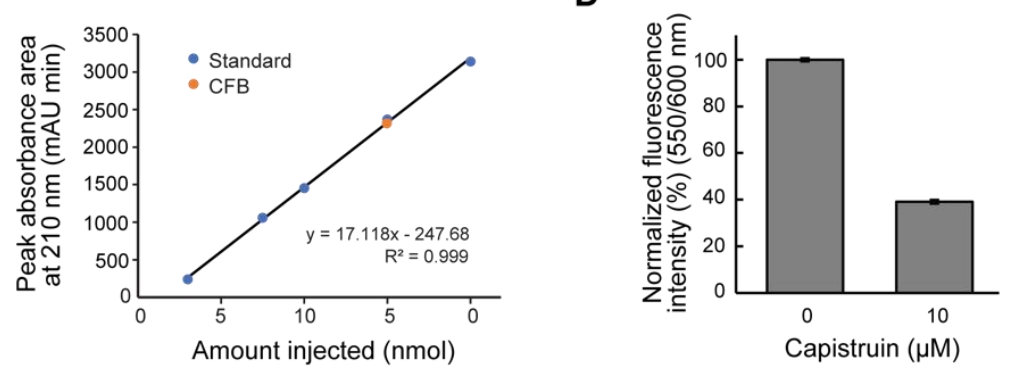

Figure S8. Purification and yield determination of capistruin produced from CFB. (A) LC-MS confirms CFB-produced capistruin is identical to an authentic sample. ${ }^{4}$ Extracted ion chromatograms for $m / z$ 1025.4 $\left([\mathrm{M}+2 \mathrm{H}]^{2+}\right.$ ion of capistruin) are shown for samples of capistruin isolated from CFB (green, top), a standard isolated from heterologous expression of capistruin (blue, middle), and a co-injection (black, bottom). (B) High-resolution tandem mass spectrum of capistruin from CFB (top) compared to the capistruin standard (bottom). (C) Quantification of capistruin from a $750 \mu \mathrm{L} \mathrm{CFB}$ reaction. The area of the $220 \mathrm{~nm}$ absorbance peak corresponding to capistruin was compared to a standard curve to determine yield. Orange circle, CFB-produced capistruin; blue circles, authentic standards. (D) CFB-produced capistruin inhibited the CFB-based production of mCherry. Error bars represent standard deviation $\left(n=2, \mathrm{p}\right.$-value $\left.=8.7 \times 10^{-5}\right)$. 


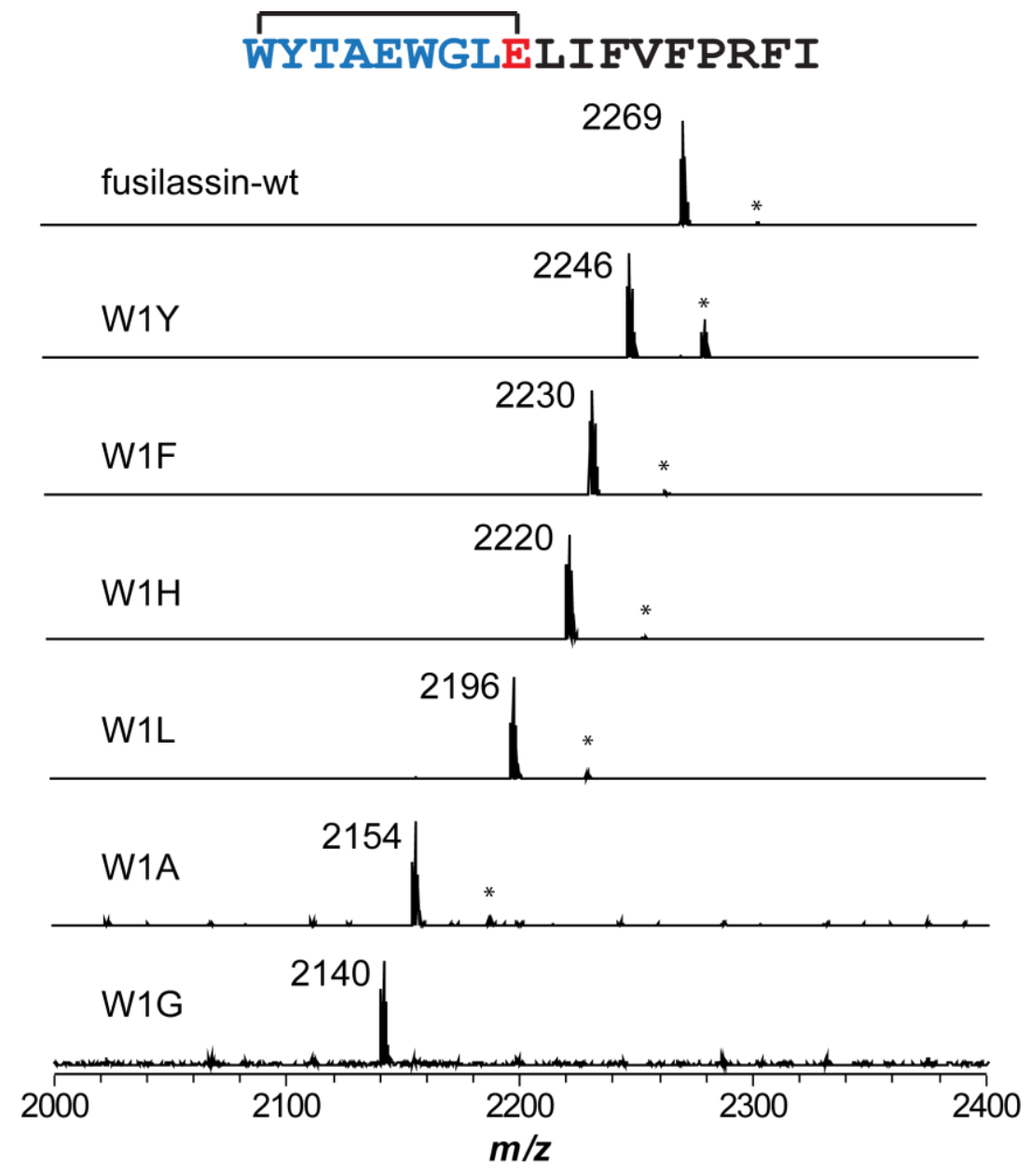

Figure S9. MALDI-TOF-MS of Fusilassin variants with Trp1 substitutions. End point MALDI-TOF-MS assay of fusilassin variants produced from CFB with different substitutions on the first position. The mass label corresponds to the $[\mathrm{M}+\mathrm{H}]^{+}$ion of the lasso peptide. ${ }^{*}$ indicates $[\mathrm{M}+\mathrm{K}]^{+}$ ion of lasso peptide. 


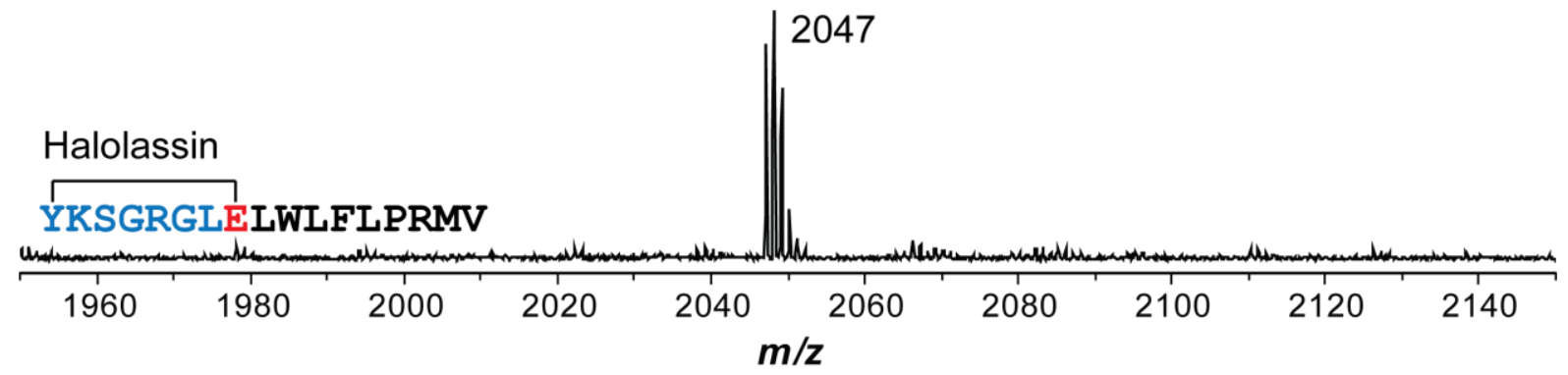

Figure S10. MALDI-TOF-MS of halolassin produced from CFB with plasmid DNA. Halolassin ( $m / z$ 2047, predicted from Thermobifida halotolerans) was produced from CFB with FusA serving as a Fus-compatible chimeric substrate and pACYC-fusC-fusEB as the DNA template for the modification enzymes. 


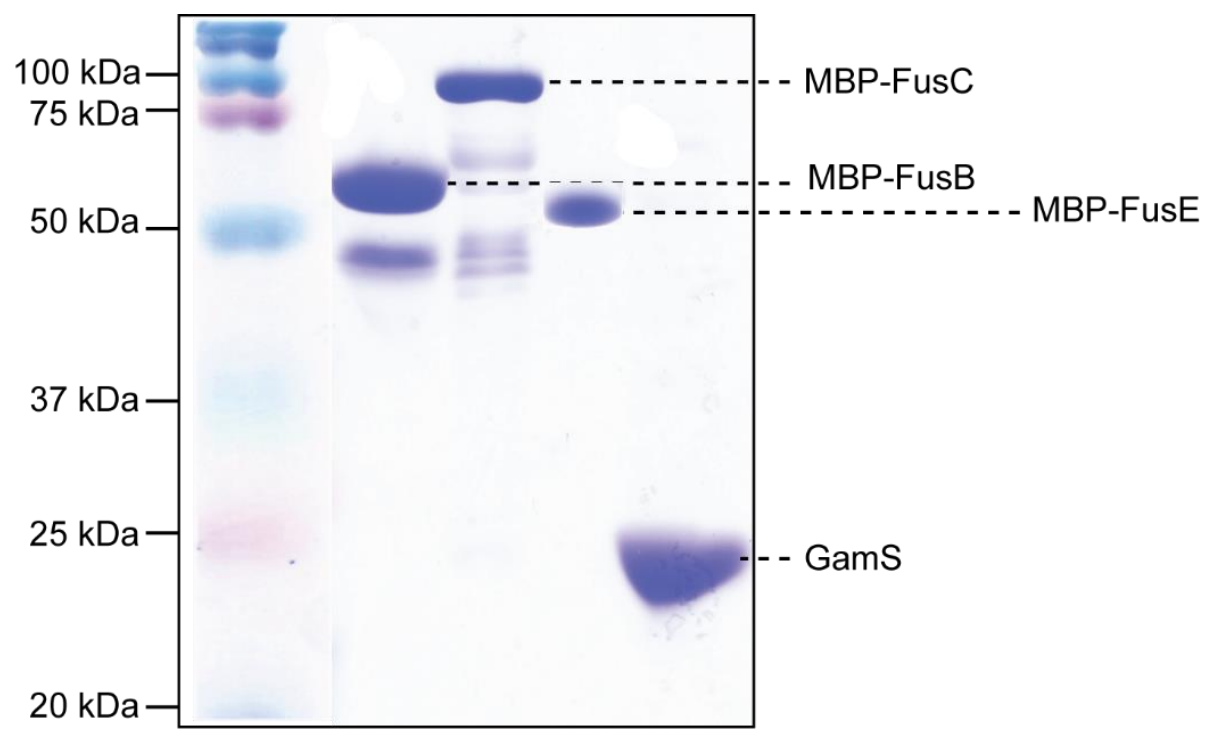

Figure S11. SDS-PAGE gel of protein used in this study. From left to right: MBP-FusB; MBP-FusC; MBP-FusE; 6xHis-GamS. All four proteins were heterologously expressed in E. coli and purified to homogeneity by amylose affinity chromatography (MBP-FusB, MBP-FusC, MBP-FusE) or Ni-NTA affinity chromatography (6xHis-GamS). 
A

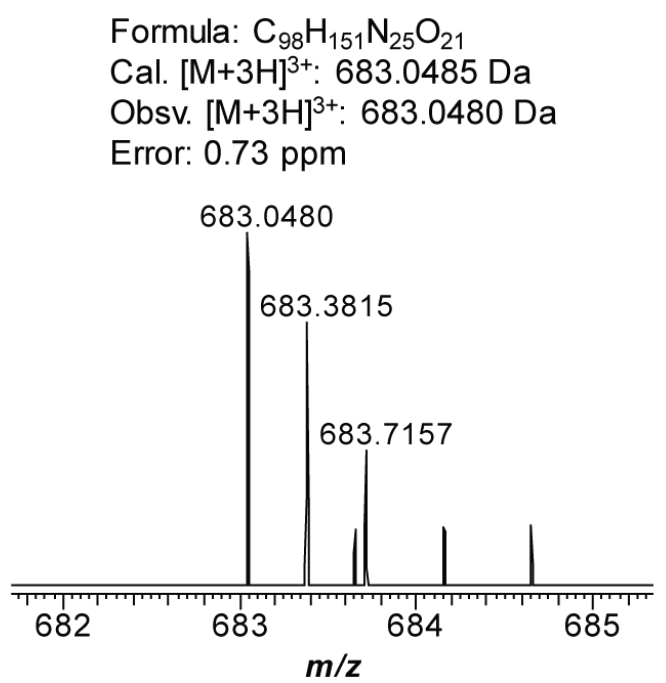

C

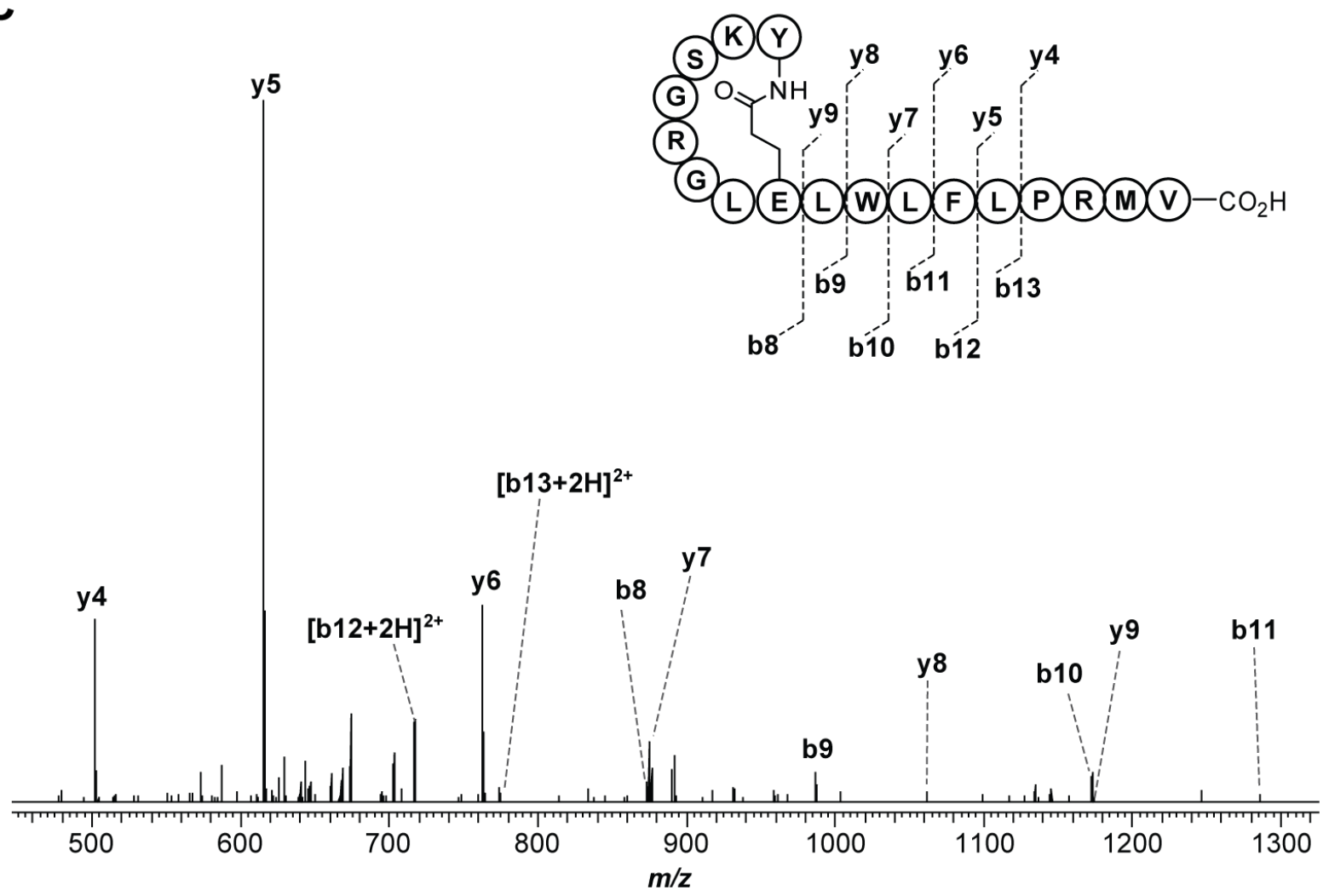

B

\begin{tabular}{|c|c|c|c|}
\hline Ion & $\begin{array}{c}\text { Calculated } \\
\text { mass (Da) }\end{array}$ & $\begin{array}{c}\text { Observed } \\
\text { mass (Da) }\end{array}$ & $\begin{array}{c}\text { Error } \\
\text { (ppm) }\end{array}$ \\
\hline b8 & 873.4583 & 873.4557 & 2.98 \\
\hline b9 & 986.5423 & 986.5410 & 1.32 \\
\hline b10 & 1172.6217 & 1172.6191 & 2.22 \\
\hline b11 & 1285.7057 & 1285.7007 & 3.89 \\
\hline$[\mathrm{b} 12+2 \mathrm{H}]^{2+}$ & 716.8904 & 716.8895 & 1.26 \\
\hline$[\mathrm{b} 13+2 \mathrm{H}]^{2+}$ & 773.4325 & 773.4316 & 1,16 \\
\hline $\mathrm{y} 4$ & 502.2812 & 502.2800 & 2.39 \\
\hline $\mathrm{y} 5$ & 615.3652 & 615.3638 & 2.28 \\
\hline $\mathrm{y} 6$ & 762.4336 & 762.4321 & 1.84 \\
\hline $\mathrm{y} 7$ & 875.5177 & 875.5158 & 2.40 \\
\hline $\mathrm{y} 8$ & 1061.5970 & 1061.5938 & 3.01 \\
\hline $\mathrm{y} 9$ & 1174.6811 & 1174.6787 & 2.04 \\
\hline
\end{tabular}

Figure S12. High-resolution and tandem MS of halolassin produced from CFB using chimeric substrate FusA LP-HalA $_{C P}$ as the DNA template. (A) High-resolution broadband spectrum of halolassin, produced from CFB with FusA $A_{L P}-H_{a l} A_{C P}$ as the DNA template and reacted with heterologously expressed and purified FusB, C, and E. (B) Mass assignment for the $\mathrm{b}^{+}$and $\mathrm{y}^{+}$ions generated from CID of halolassin. (C) Tandem MS spectrum of halolassin, consistent with a Tyr1Glu8 macrolactam. 
A

Formula: $\mathrm{C}_{116} \mathrm{H}_{165} \mathrm{~N}_{25} \mathrm{O}_{23}$

Cal. $[\mathrm{M}+2 \mathrm{H}]^{2+}: 1139.1328 \mathrm{Da}$

Obsv. $[\mathrm{M}+2 \mathrm{H}]^{2+:}: 1139.1317 \mathrm{Da}$

Error: $0.97 \mathrm{ppm}$

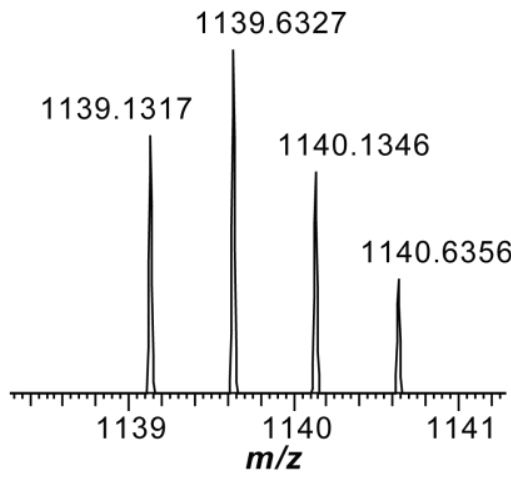

B

\begin{tabular}{|c|c|c|c|}
\hline Ion & $\begin{array}{c}\text { Calculated } \\
\text { mass (Da) }\end{array}$ & $\begin{array}{c}\text { Observed } \\
\text { mass (Da) }\end{array}$ & $\begin{array}{c}\text { Error } \\
\text { (ppm) }\end{array}$ \\
\hline b9 & 1080.5631 & 1080.5602 & 2.68 \\
\hline b10 & 1193.6471 & 1193.6444 & 2.18 \\
\hline b11 & 1356.7105 & 1356.7063 & 3.10 \\
\hline b12 & 1469.7945 & 1469.7915 & 2.04 \\
\hline b13 & 1582.8786 & 1582.8748 & 2.40 \\
\hline b14 & 1730.9470 & 1730.9447 & 1.33 \\
\hline y4 & 548.3197 & 548.3183 & 2.55 \\
\hline y5 & 695.3881 & 695.3863 & 2.59 \\
\hline y6 & 808.4721 & 808.4703 & 2.23 \\
\hline y7 & 921.5562 & 921.5541 & 2.28 \\
\hline y8 & 1084.6195 & 1084.6167 & 2.58 \\
\hline y9 & 1197.7036 & 1197.7007 & 2.42 \\
\hline
\end{tabular}

C

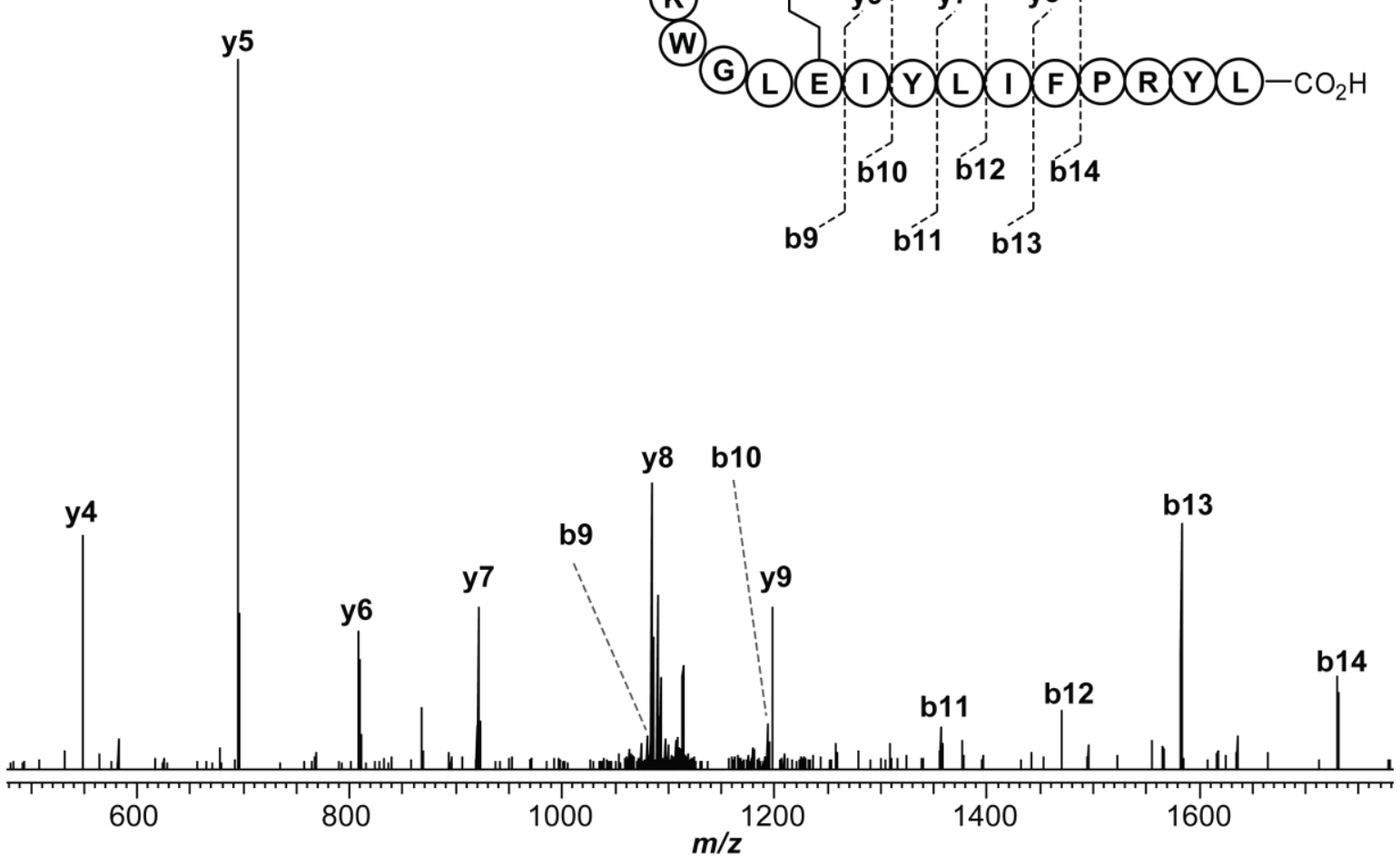

Figure S13. High-resolution and tandem MS of cellulassin produced from CFB using chimeric substrate FusA LP-CelAcP as the DNA template. (A) High-resolution broadband spectrum of cellulassin, produced from CFB with FusA heterologously expressed and purified FusB, C, and E. (B) Mass assignment for the $\mathrm{b}^{+}$and $\mathrm{y}^{+}$ions generated from CID of cellulassin. (C) Tandem MS spectrum of cellulassin, consistent with a Trp1Glu9 macrolactam. 


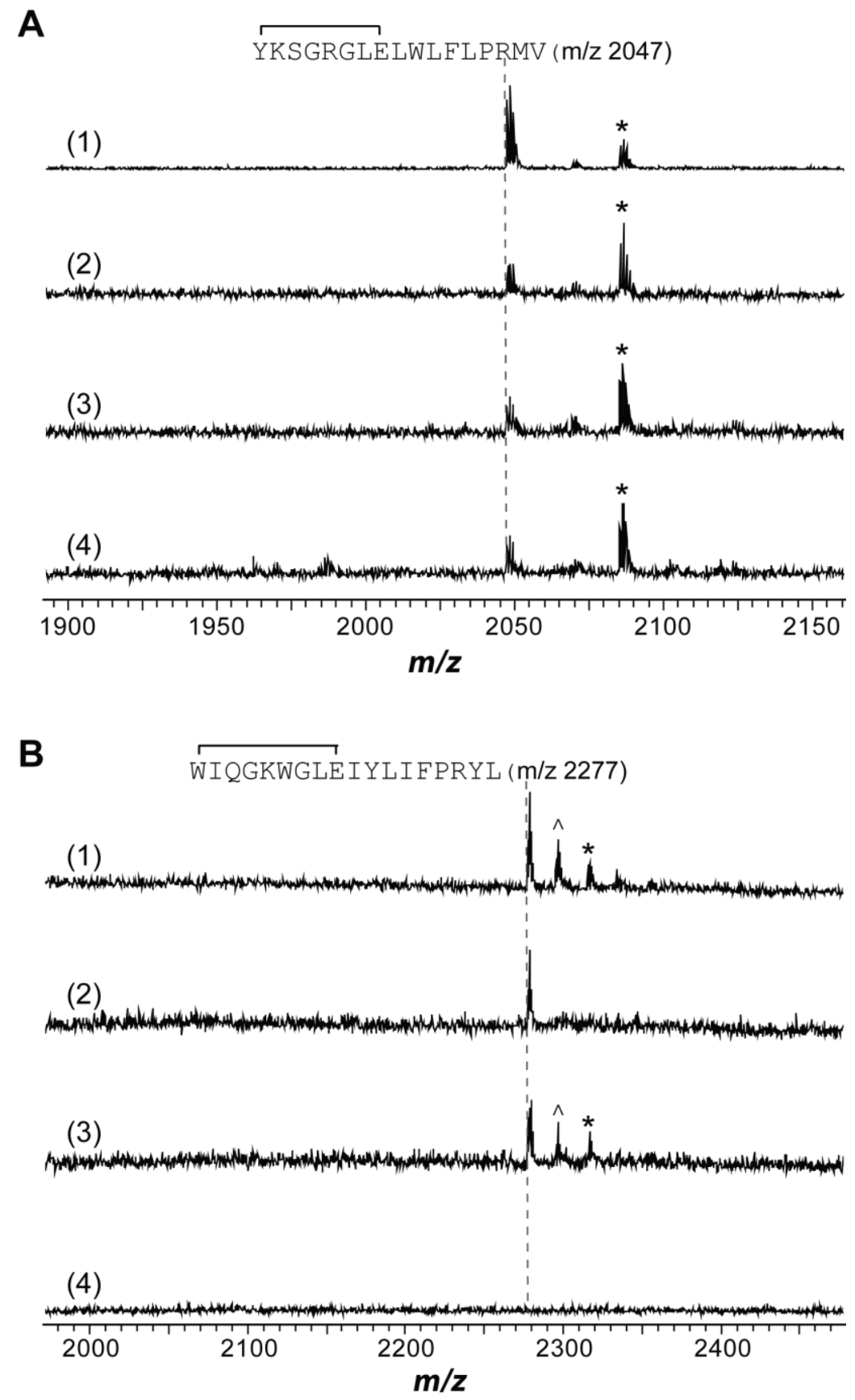

Figure S14. Carboxypeptidase Y treatment of halolassin and cellulassin produced with chimeric substrates from CFB. Halolassin and cellulassin were produced through CFB using chimeric precursor peptides as the DNA template (1). The produced lasso peptides were treated under 3

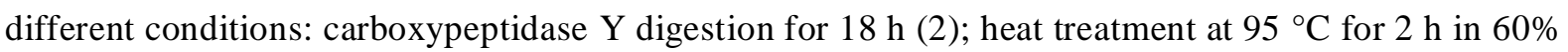
$\mathrm{ACN}$ (3); heat treatment at $95{ }^{\circ} \mathrm{C}$ for $2 \mathrm{~h}$ in $60 \% \mathrm{ACN}$ followed by carboxypeptidase $\mathrm{Y}$ digestion for $18 \mathrm{~h}$ at room temperature (4). The presence/absence of intact lasso peptide upon treatment under condition (4) and the presence of intact lasso peptide under conditions (2) and (3) indicate the threaded isomer of a lasso peptide. ${ }^{\wedge}$ indicates the uncyclized linear core peptide. ${ }^{*}$ indicates the $[\mathrm{M}+\mathrm{K}]^{+}$ion of the lasso peptide. 

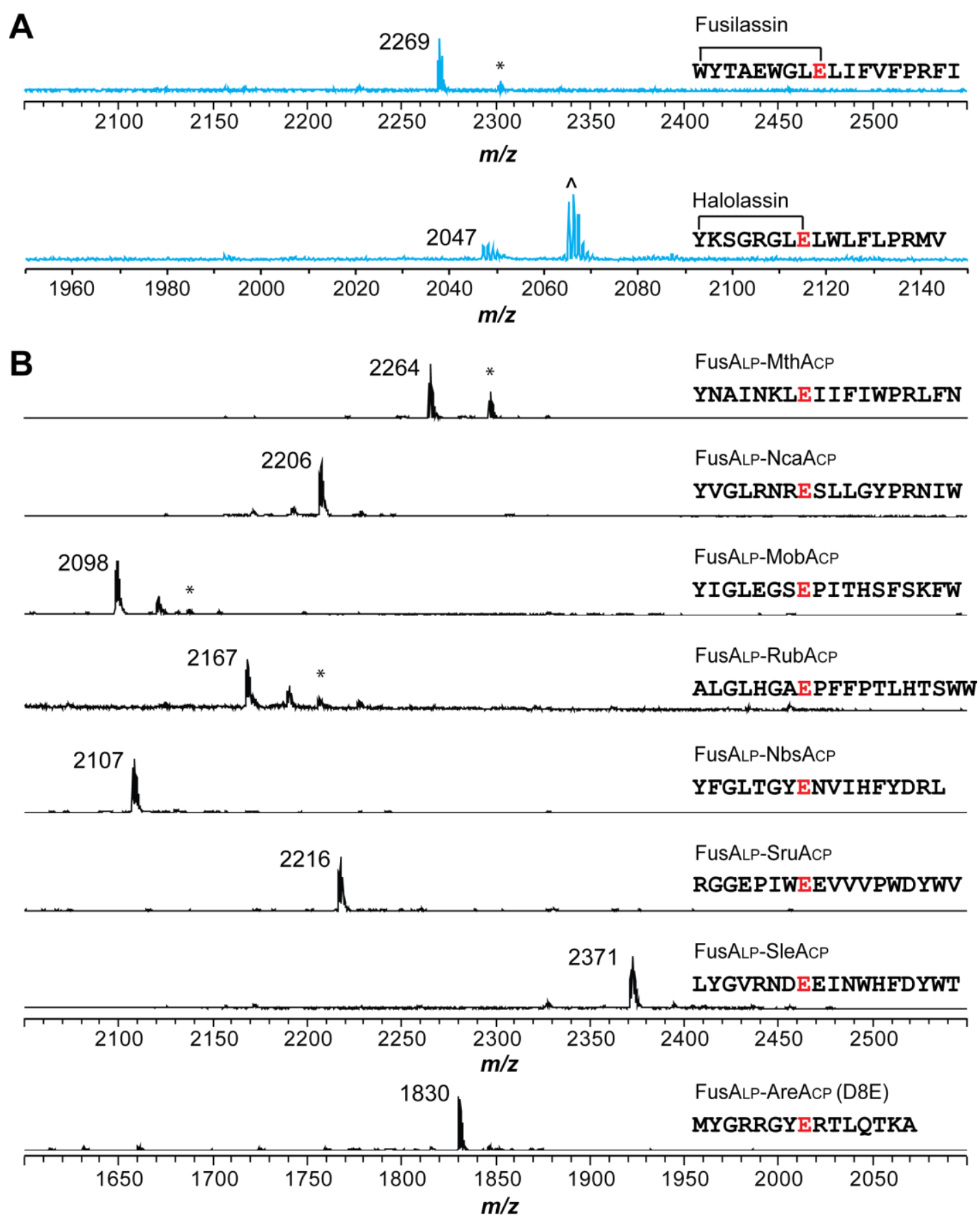

Figure S15. Chimeric substrate processing in PURExpress reactions. (A) Wildtype FusA and Fus $A_{L P}-H_{a l} A_{C P}$ were synthesized using PURExpress and treated with purified FusB, FusC, and FusE. Analysis by MALDI-TOF-MS demonstrated the production of mature lasso peptides (blue) fusilassin $\left(\mathrm{m} / \mathrm{z}\right.$ 2269), and to a lesser extent, halolassin $\left(\mathrm{m} / \mathrm{z} 2\right.$ 2047). Mass labels corresponds to the $[\mathrm{M}+\mathrm{H}]^{+}$ion of the lasso peptide. ${ }^{\wedge}$ indicates the uncyclized linear core peptide. $*$ indicates the $[\mathrm{M}+\mathrm{K}]^{+}$ion of the lasso peptide. (B) Eight chimeric substrates were produced by PURExpress and reacted with purified FusB, FusC, and FusE. MALDI-TOF-MS detected masses corresponding to the $[\mathrm{M}+\mathrm{H}]^{+}$ions for the linear core peptides, indicating all substrates were processed by FusB/E but none were processed by FusC. * indicates $[\mathrm{M}+\mathrm{K}]^{+}$ion for the linear core peptide. 
A
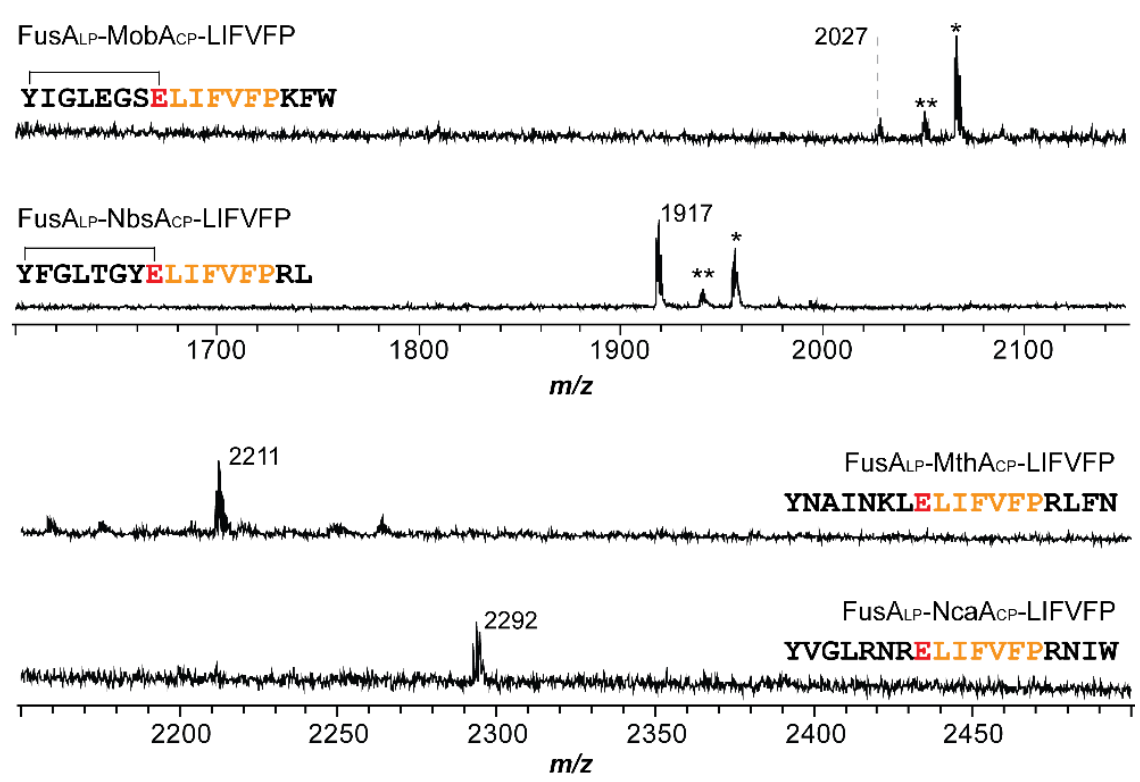

B
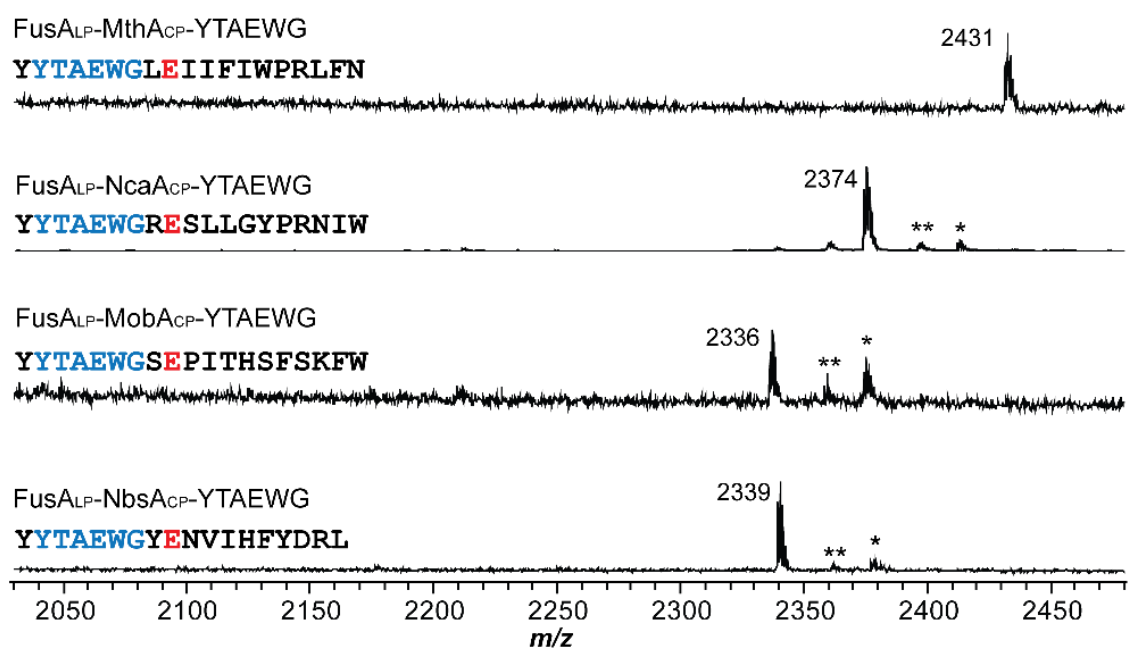

Figure S16. Replacement of the ring/loop sequences of chimeric substrates with FusA ring/loop sequence. (A) Four new chimeric substrates, in which the loop region of the core sequence was replaced with FusA loop (orange), were produced by CFB and reacted with purified FusB, FusC, and FusE. Cyclized FusA $A_{L P}-M o b A_{C P}-L I F V F P(m / z, 2027)$ and FusA $A_{L P}-N b s A_{C P}-L I F V F P(m / z, 1917)$ were detected, while the other two failed to cyclize. FusA $A_{L P}-M A_{\text {th }} A_{C P}$-LIFVFP and FusA $A_{L P}-N_{C a} A_{C P}-L I F V F P$ were produced through PURExpress and reacted with FusB, FusC, and FusE. Their corresponding linear core peptides were detected by MALDI $(\mathrm{m} / \mathrm{z}$ of 2211 and 2292, respectively). (B) Four additional chimeric substrates, in which a portion of the ring region was replaced with FusA ring sequence (blue), were also prepared. All four new chimeric substrates failed to cyclize in CFB and their linear core peptides were produced in PURExpress and detected by MALDI $(\mathrm{m} / \mathrm{z}$ of 2431,2374 , 2336 , and 2339 , respectively). $*$ indicates the $[\mathrm{M}+\mathrm{K}]^{+}$ion of cyclized lasso peptide/uncyclized core peptide, ${ }^{* *}$ indicates the $[\mathrm{M}+\mathrm{Na}]^{+}$ion of cyclized lasso peptide/uncyclized core peptide. 


\section{A}
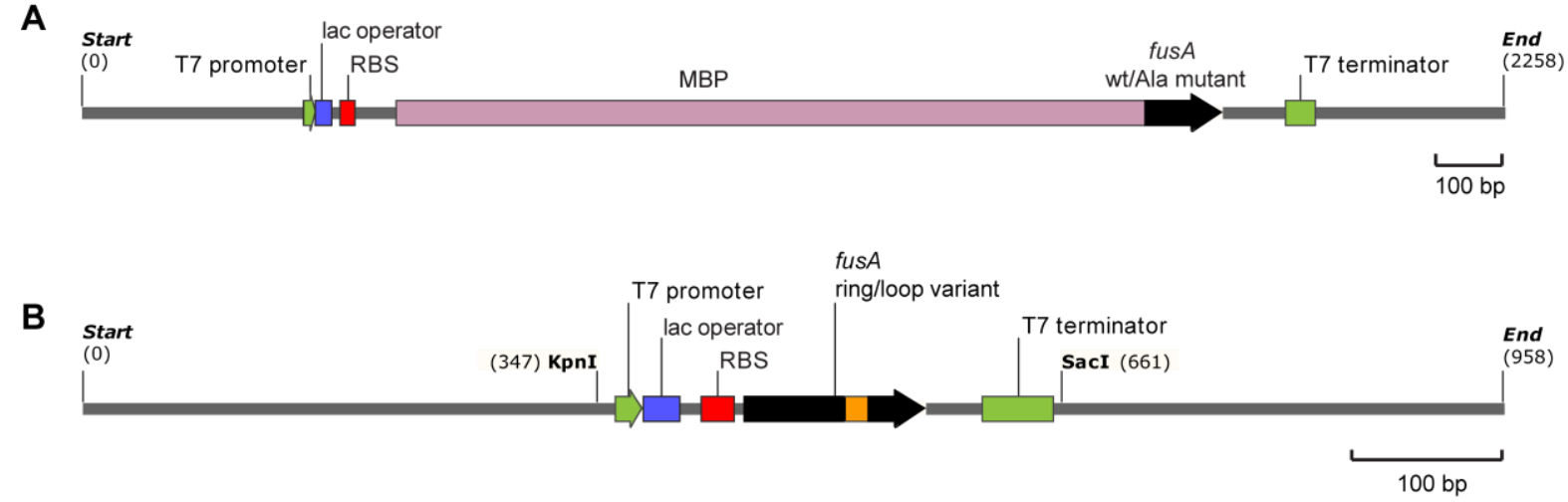

Figure S17. Linear DNA template used in CFB reactions. (A) The linear DNA template of fusA wild type and Ala-substituted variants used in CFB reactions. (B) The linear DNA template of fusA ring/loop variants used for $\mathrm{CFB}$ reactions. $\mathrm{T} 7$ promotor and $\mathrm{T} 7$ terminator is shown in green; lac operators are shown in blue; RBSs are shown in red; fusA genes are shown in black; MBP, maltose-binding protein, is shown in pink. 


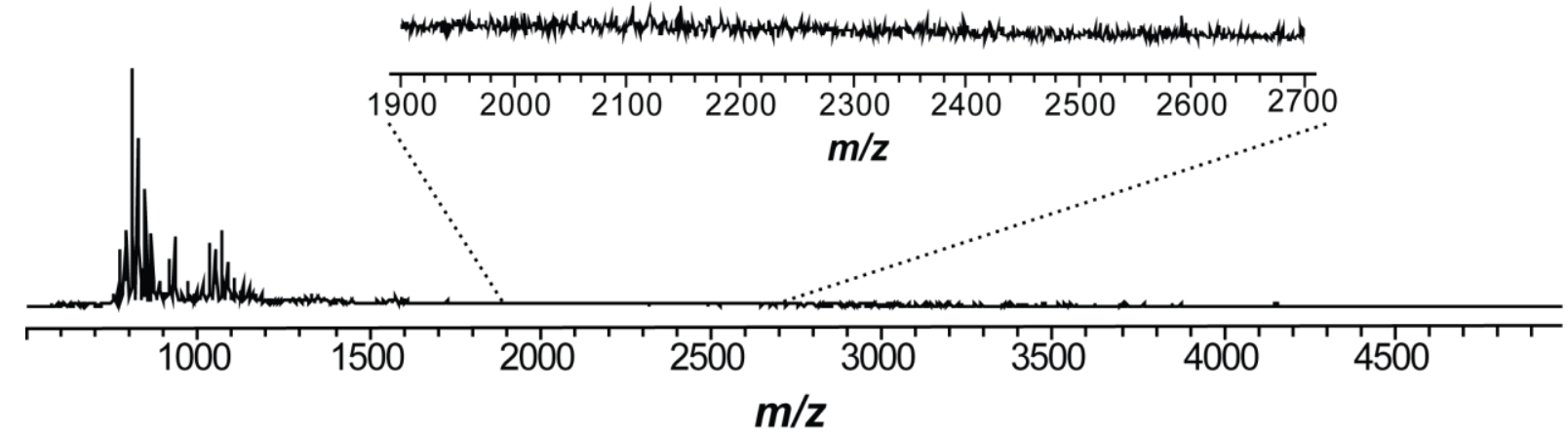

Figure S18. CFB reaction without DNA template. The control reaction was conducted the same way as other CFB reactions including fusilassin biosynthetic proteins, but with no DNA template. No signal was present in the mass range of 1900-2700 Da. 


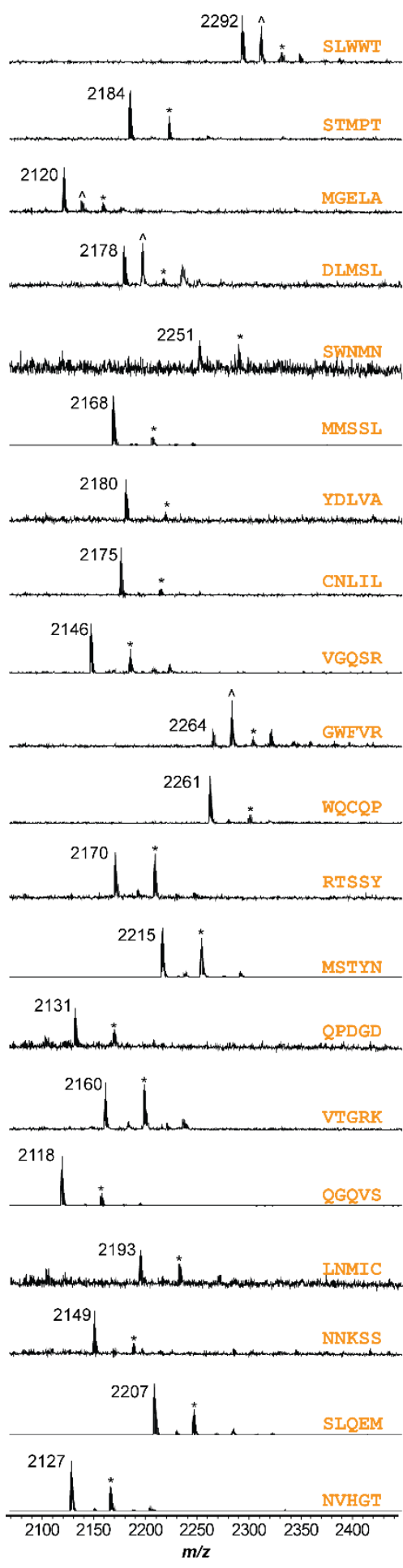

\section{WXXXXXGLELIFVFPRFI}
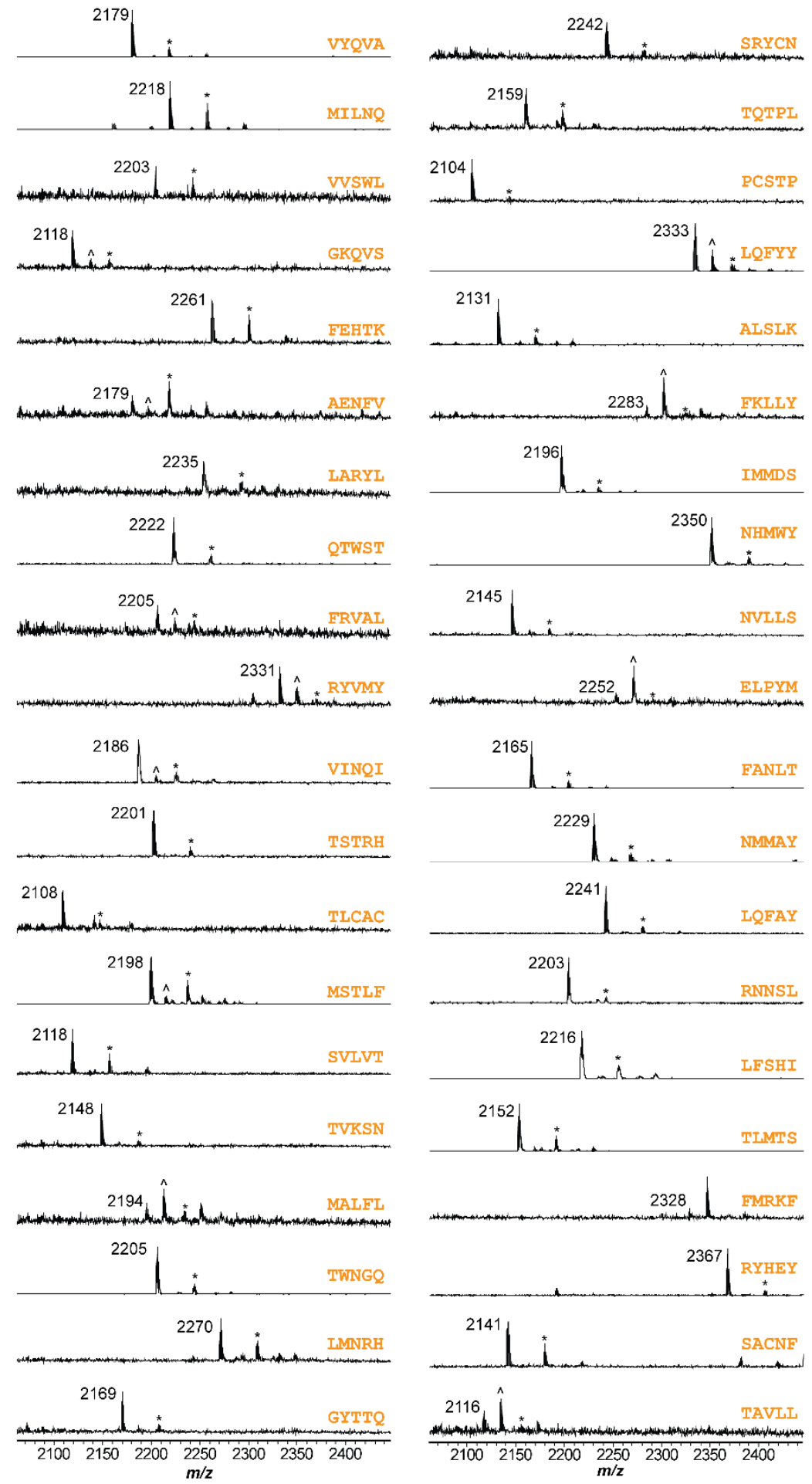

Figure S19. MALDI-TOF mass spectra of the fusilassin ring variants produced from CFB. Fusilassin variants (270 out of 513; an additional 10 are shown in main text Figure 4) detected by MALDI-TOF-MS are shown. The DNA encoding each ring variant product was subjected to Sanger sequencing. Orange depicts the varied sequence of positions 2-6 of the FusA core region. The mass label corresponds to the $[\mathrm{M}+\mathrm{H}]^{+}$ion of the lasso peptide. * indicates $[\mathrm{M}+\mathrm{K}]^{+}$ion of the lasso peptide. $\wedge$ indicates the uncyclized linear core peptide. Figure continues onto the next 4 pages. 


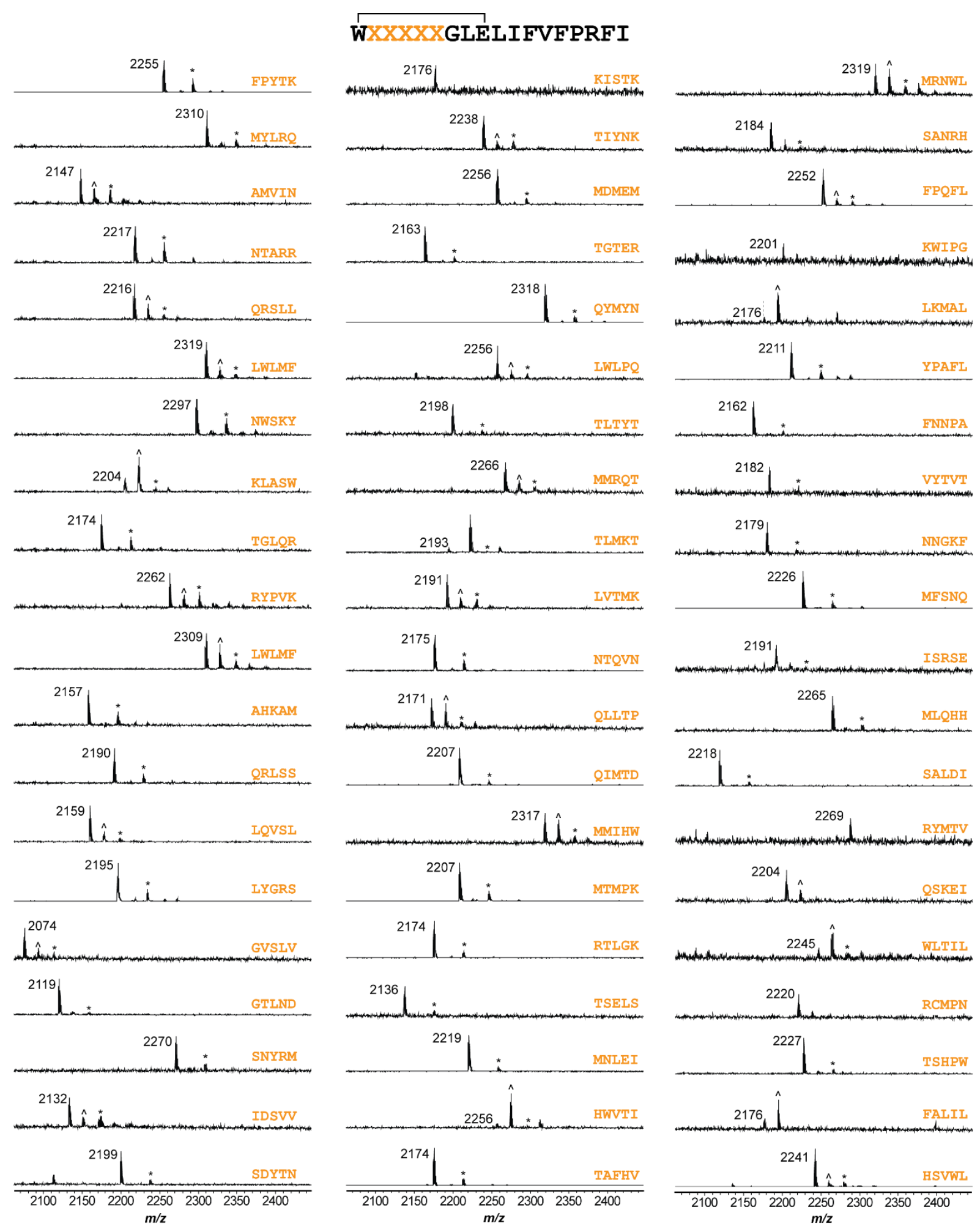

Figure S19. Continued from previous page ( 2 of 5 ) 


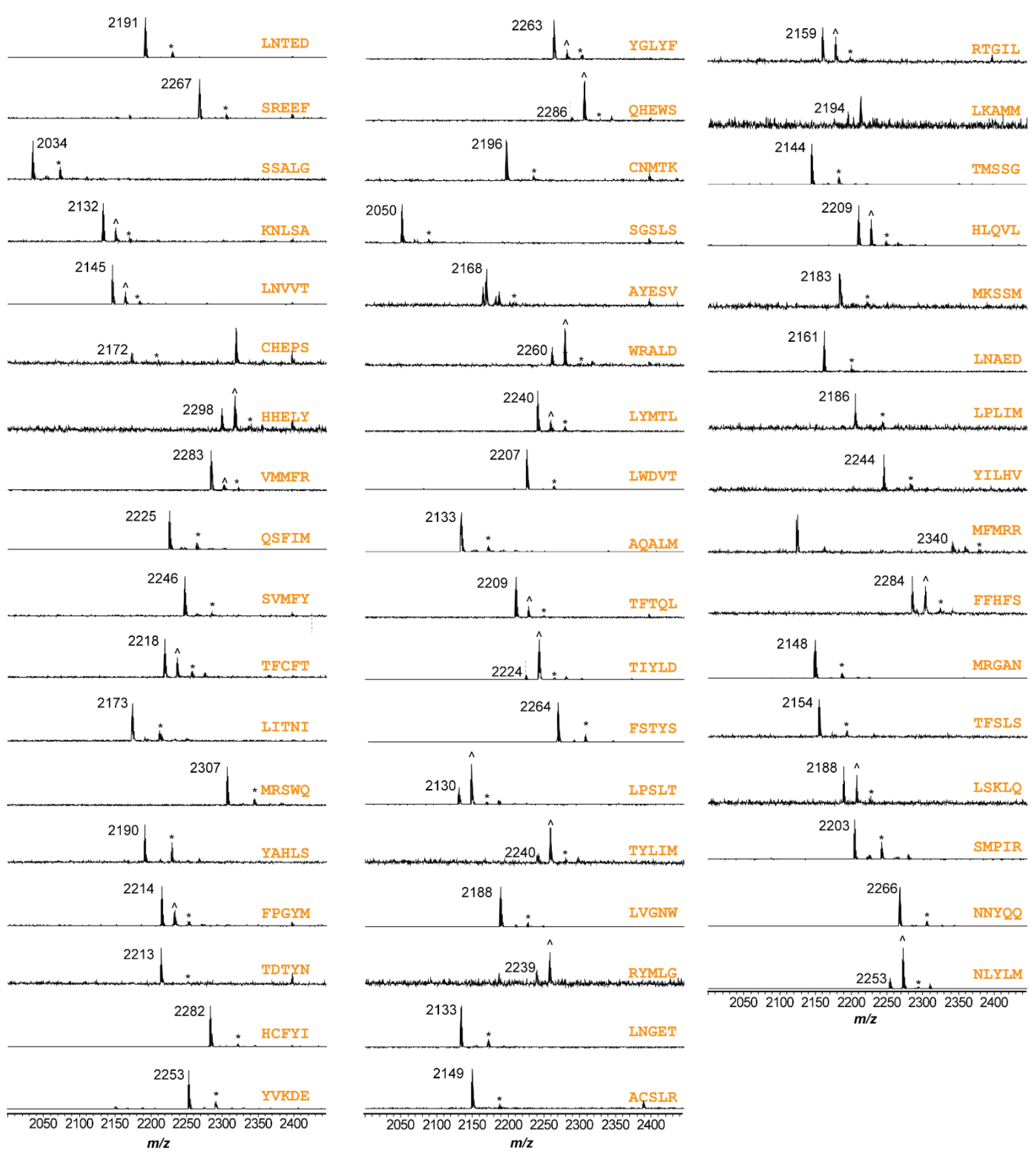

Figure S19. Continued from previous page ( 3 of 5 ) 


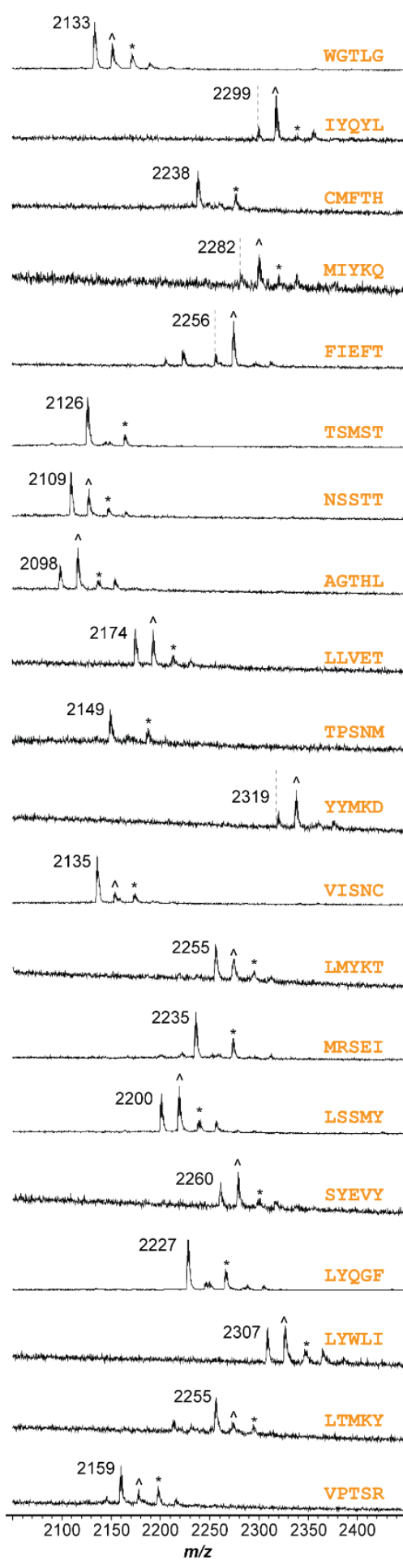

\section{WXXXXXGLELIFVFPRFI}
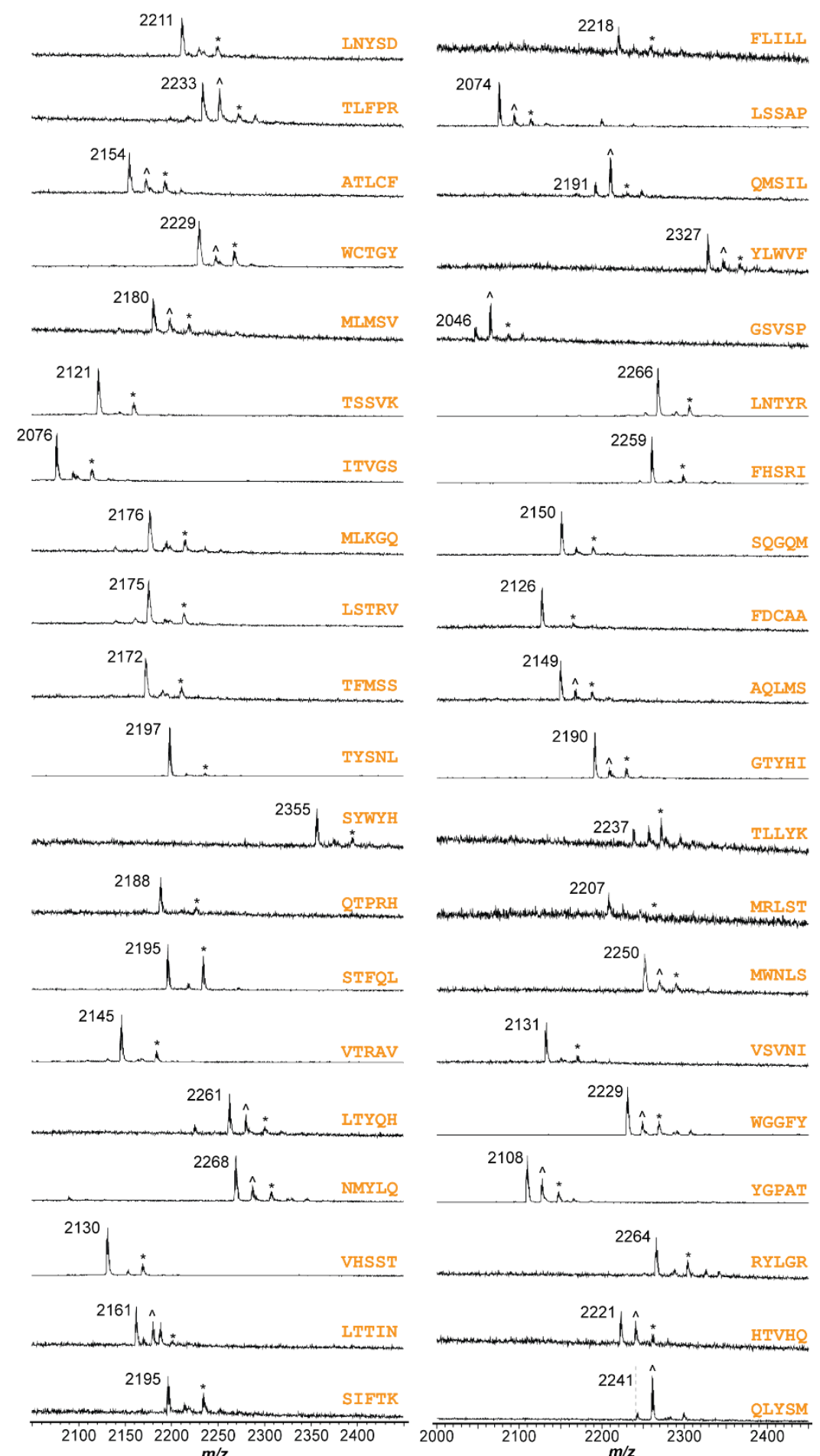

Figure S19. Continued from previous page (4 of 5) 


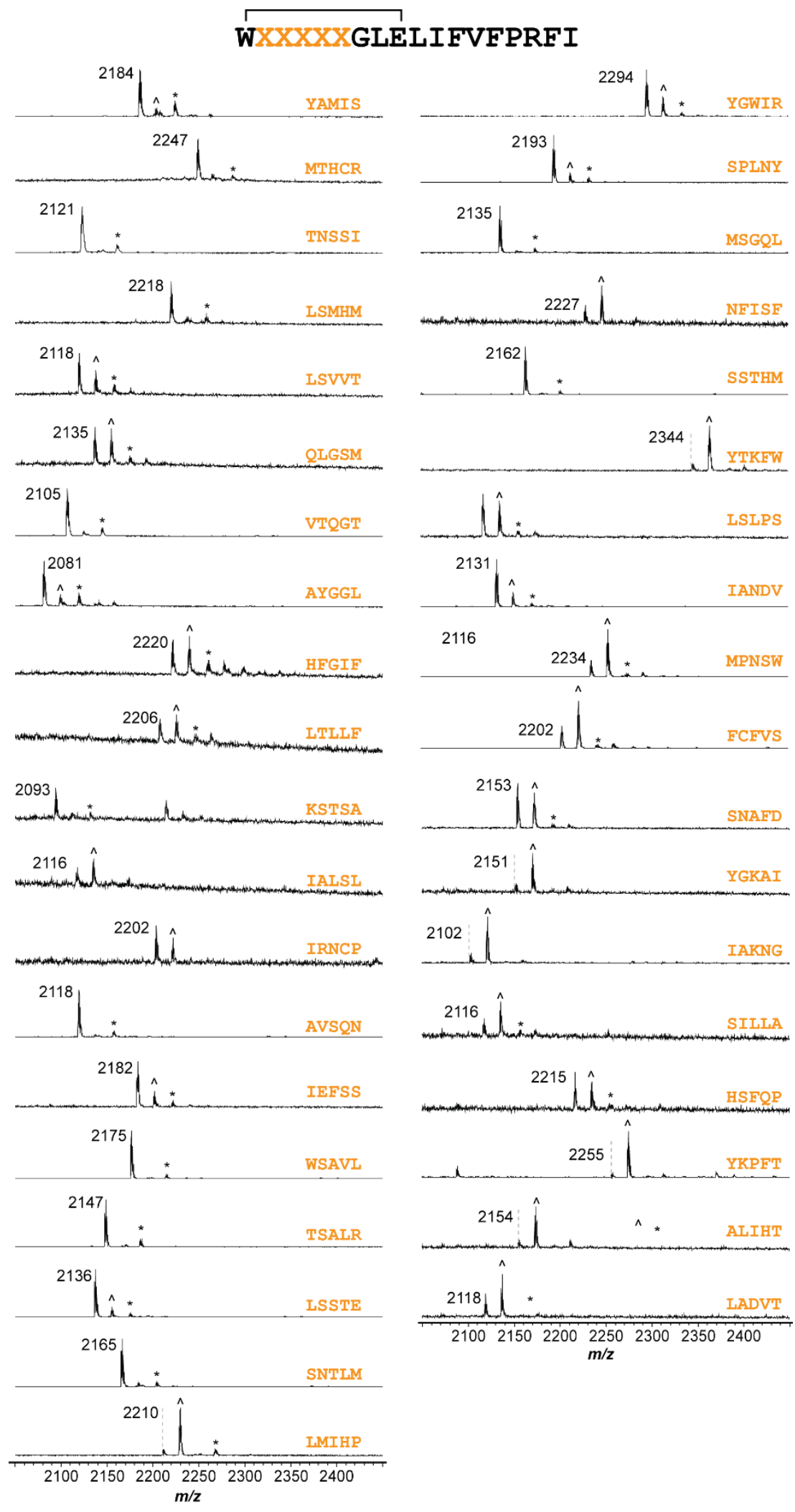

Figure S19. Continued from previous page (5 of 5) 
A

Formula: $\mathrm{C}_{104} \mathrm{H}_{151} \mathrm{~N}_{23} \mathrm{O}_{22}$

Cal. $[\mathrm{M}+2 \mathrm{H}]^{2+}: 1038.0780 \mathrm{Da}$

Obsv. $[\mathrm{M}+2 \mathrm{H}]^{2+:}: 1038.0763 \mathrm{Da}$

Error: $1.64 \mathrm{ppm}$

1038.5779

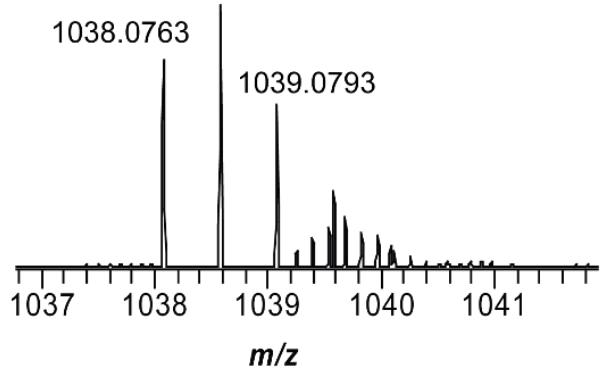

C

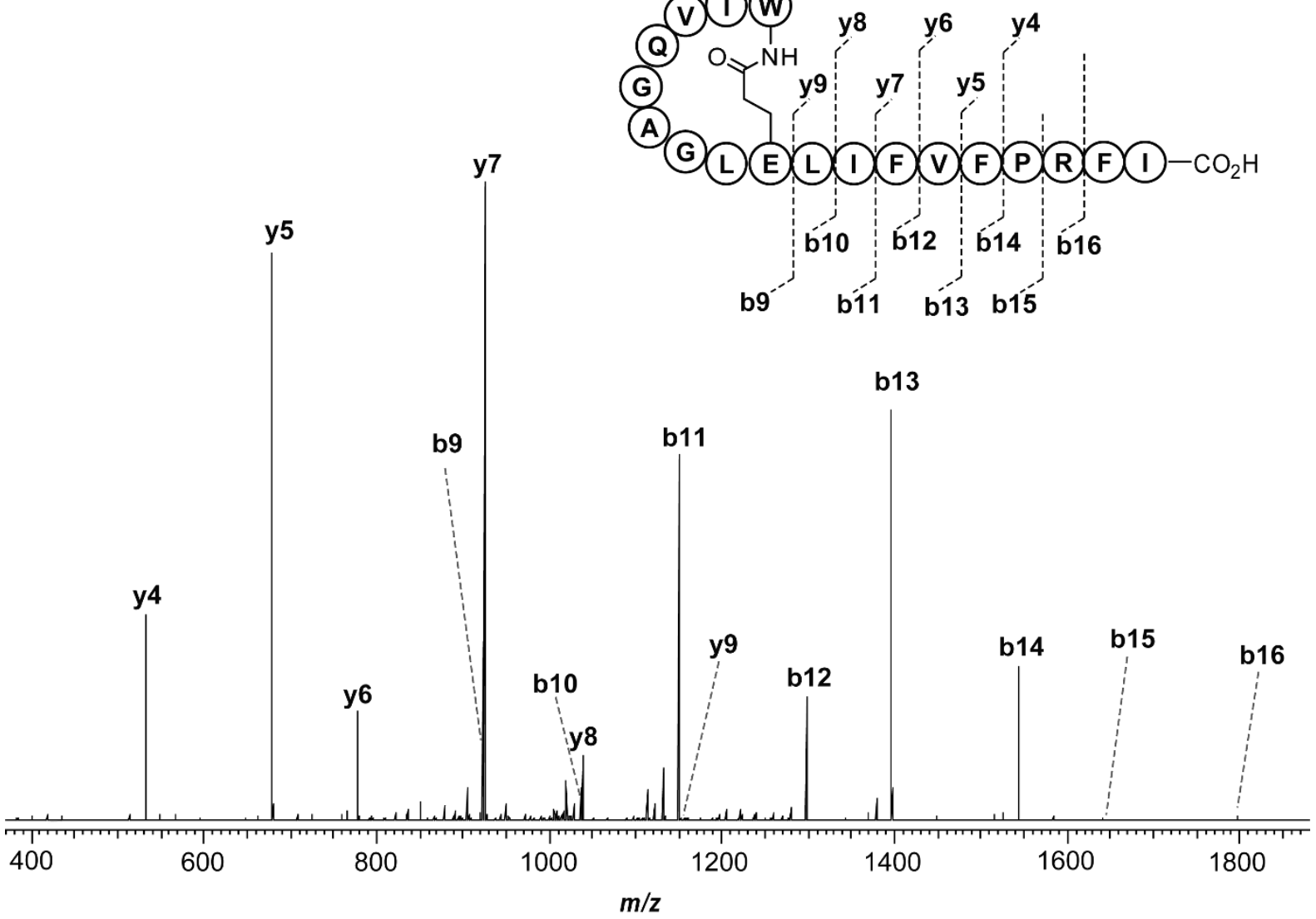

Figure S20. High-resolution and tandem MS of TVQGA variant produced from CFB. (A) High-resolution broadband spectrum of fusilassin TVQGA variant, produced from CFB. (B) Mass assignment for the $\mathrm{b}^{+}$and $\mathrm{y}^{+}$ions generated from CID of fusilassin TVQGA variant. (C) Tandem MS spectrum of fusilassin TVQGA variant, consistent with a Trp1-Glu9 macrolactam linkage. 
A

Formula: $\mathrm{C}_{107} \mathrm{H}_{148} \mathrm{~N}_{22} \mathrm{O}_{24}$ Cal. $[\mathrm{M}+2 \mathrm{H}]^{2+}: 1063.5591 \mathrm{Da}$ Obsv. $[\mathrm{M}+2 \mathrm{H}]^{2+}: 1063.5587 \mathrm{Da}$ Error: $0.38 \mathrm{ppm}$

1064.0602

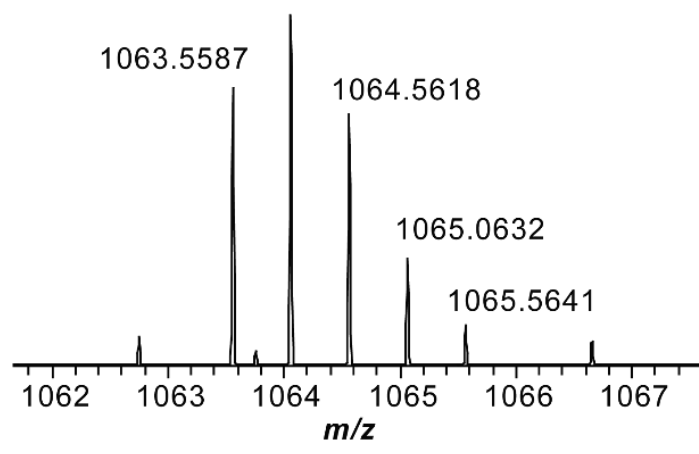

B

\begin{tabular}{|c|c|c|c|}
\hline Ion & $\begin{array}{c}\text { Calculated } \\
\text { mass (Da) }\end{array}$ & $\begin{array}{c}\text { Observed } \\
\text { mass (Da) }\end{array}$ & $\begin{array}{c}\text { Error } \\
\text { (ppm) }\end{array}$ \\
\hline b9 & 975.4212 & 975.4202 & 1.03 \\
\hline b10 & 1088.5042 & 1088.5039 & 0.28 \\
\hline b11 & 1201.5893 & 1201.5879 & 1.17 \\
\hline b12 & 1348.6578 & 1348.6564 & 1.04 \\
\hline b13 & 1447.7262 & 1447.7253 & 0.62 \\
\hline b14 & 1594.7946 & 1594.7936 & 0.63 \\
\hline y4 & 532.3247 & 532.3240 & 2.44 \\
\hline y5 & 679.3932 & 679.3923 & 2.21 \\
\hline y6 & 778.4616 & 778.4606 & 2.06 \\
\hline y7 & 925.5300 & 925.5289 & 1.84 \\
\hline y8 & 1038.6140 & 1038.6128 & 2.12 \\
\hline y9 & 1151.6981 & 1151.6968 & 2.52 \\
\hline
\end{tabular}

\section{(F)}
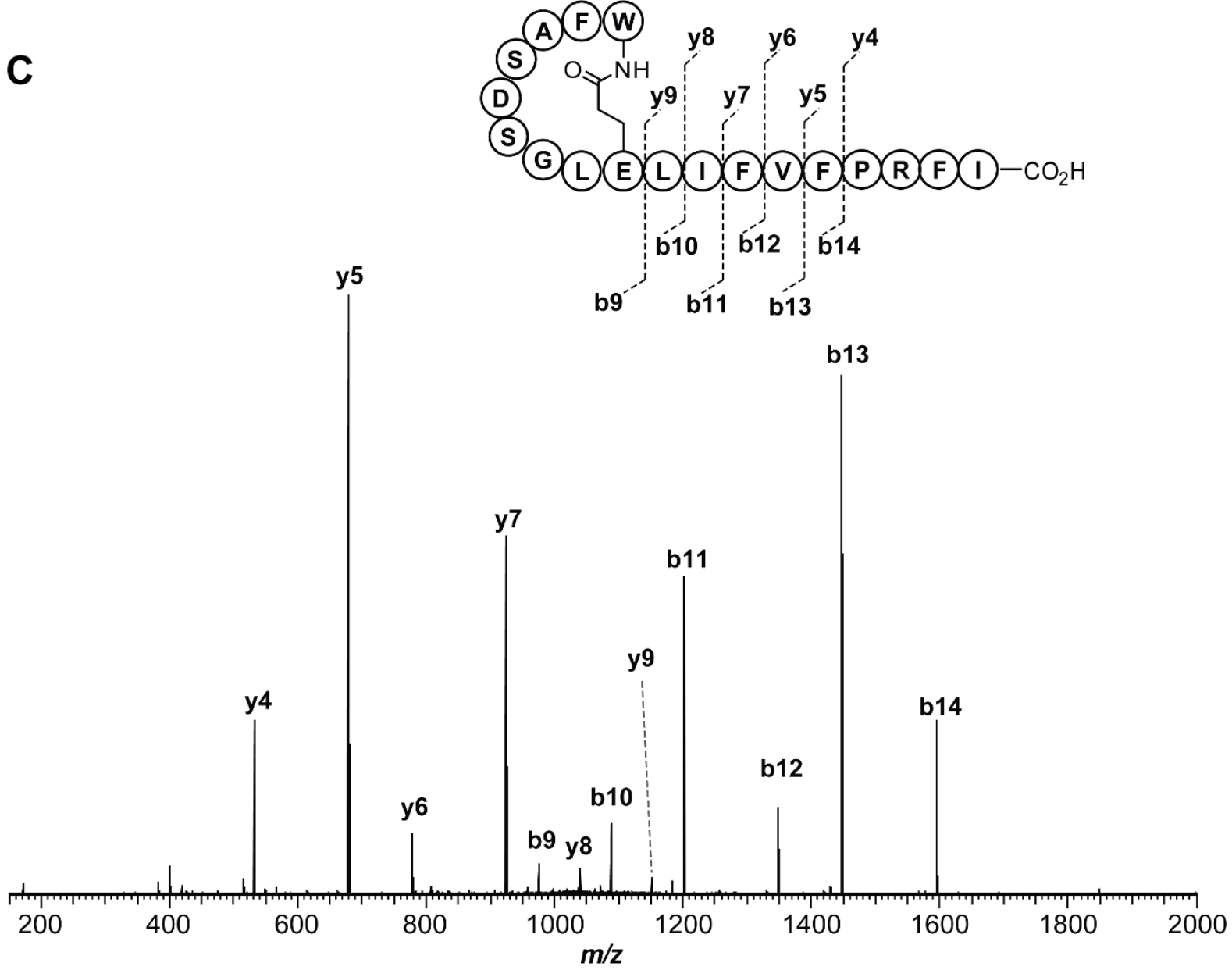

Figure S21. High-resolution and tandem MS of FASDS variant produced from CFB. (A) High-resolution broadband spectrum of fusilassin FASDS variant, produced from CFB. (B) Mass assignment for the $\mathrm{b}^{+}$and $\mathrm{y}^{+}$ions generated from CID of fusilassin FASDS variant. (C) Tandem MS spectrum of fusilassin FASDS variant, consistent with a Trp1-Glu9 macrolactam linkage. 
A

Formula: $\mathrm{C}_{109} \mathrm{H}_{155} \mathrm{~N}_{23} \mathrm{O}_{22}$

Cal. $[\mathrm{M}+2 \mathrm{H}]^{2+}: 1070.0931 \mathrm{Da}$

Obsv. $[\mathrm{M}+2 \mathrm{H}]^{2+}: 1070.0928 \mathrm{Da}$

Error: $0.28 \mathrm{ppm}$

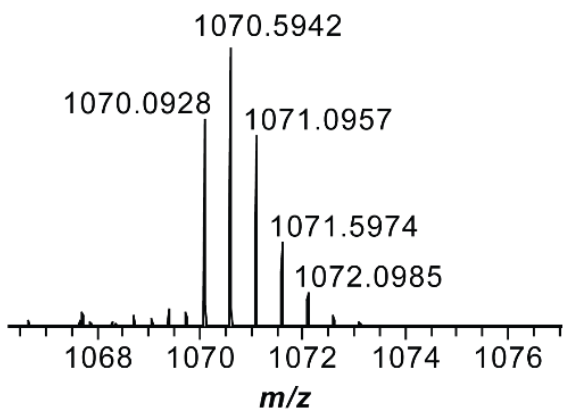

B

\begin{tabular}{|c|c|c|c|}
\hline Ion & $\begin{array}{c}\text { Calculated } \\
\text { mass (Da) }\end{array}$ & $\begin{array}{c}\text { Observed } \\
\text { mass (Da) }\end{array}$ & $\begin{array}{c}\text { Error } \\
\text { (ppm) }\end{array}$ \\
\hline b9 & 988.4892 & 988.4881 & 1.11 \\
\hline b10 & 1101.5733 & 1101.5729 & 0.36 \\
\hline b11 & 1214.6574 & 1214.6561 & 1.07 \\
\hline b12 & 1361.7258 & 1361.7242 & 1.22 \\
\hline b13 & 1460.7942 & 1460.7936 & 0.41 \\
\hline b14 & 1607.8626 & 1607.8611 & 0.93 \\
\hline y4 & 532.3247 & 532.3240 & 1.31 \\
\hline y5 & 679.3932 & 679.3923 & 1.32 \\
\hline y6 & 778.4616 & 778.4606 & 1.28 \\
\hline y7 & 925.5300 & 925.5289 & 1.19 \\
\hline y8 & 1038.6140 & 1038.6127 & 1.25 \\
\hline y9 & 1151.6981 & 1151.6968 & 1.13 \\
\hline
\end{tabular}

C

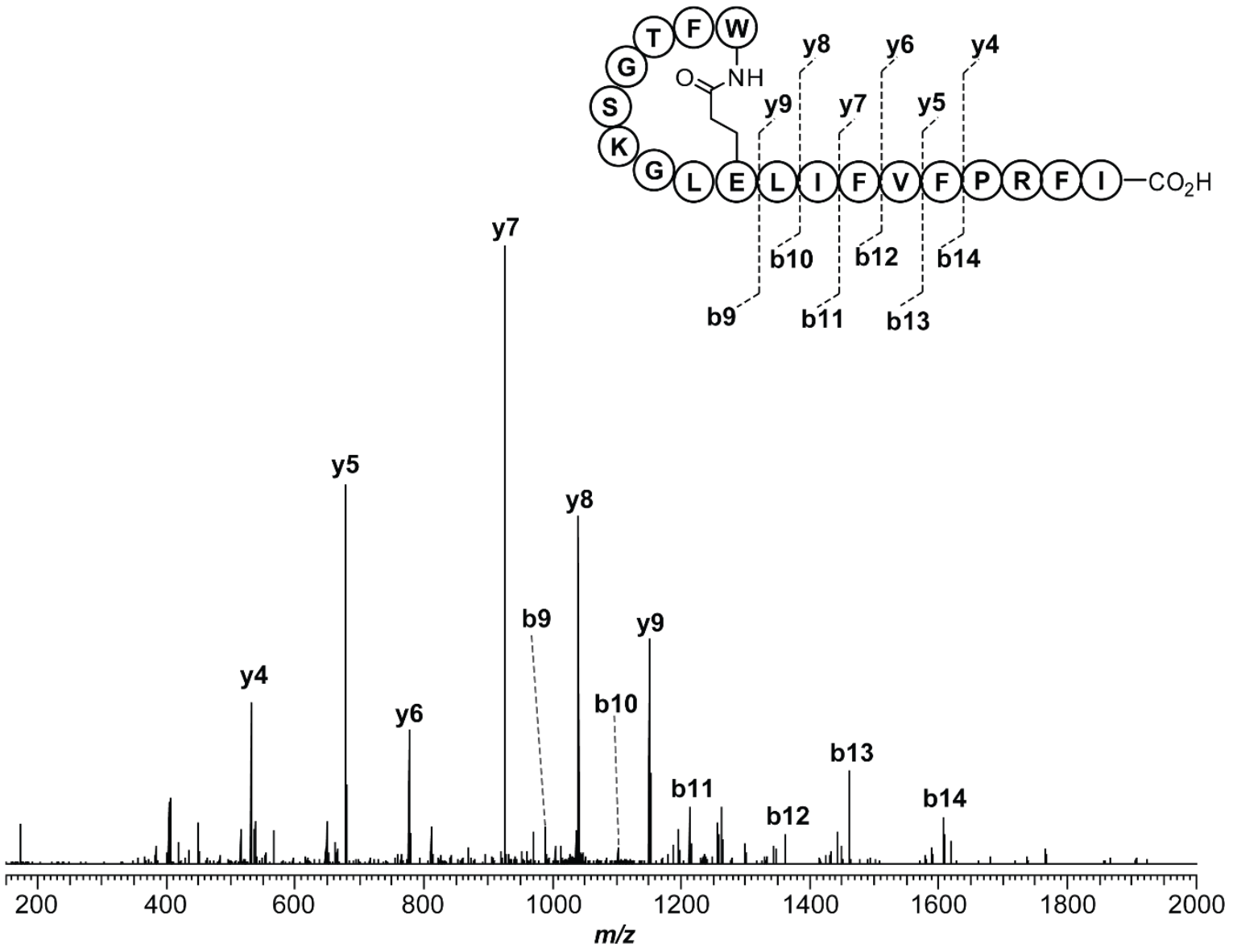

Figure S22. High-resolution and tandem MS of FTGSK variant produced from CFB. (A) High-resolution broadband spectrum of fusilassin FTGSK variant, produced from CFB. (B) Mass assignment for the $\mathrm{b}^{+}$and $\mathrm{y}^{+}$ions generated from th CID of fusilassin FTGSK variant. (C) Tandem MS spectrum of fusilassin FTGSK variant, consistent with a Trp1-Glu9 macrolactam linkage. 
A

Formula: $\mathrm{C}_{108} \mathrm{H}_{160} \mathrm{~N}_{22} \mathrm{O}_{22} \mathrm{~S}$

Cal. $[\mathrm{M}+2 \mathrm{H}]^{2+}: 1075.5972 \mathrm{Da}$

Obsv. $[\mathrm{M}+2 \mathrm{H}]^{2+}: 1075.5975 \mathrm{Da}$

Error: $0.15 \mathrm{ppm}$

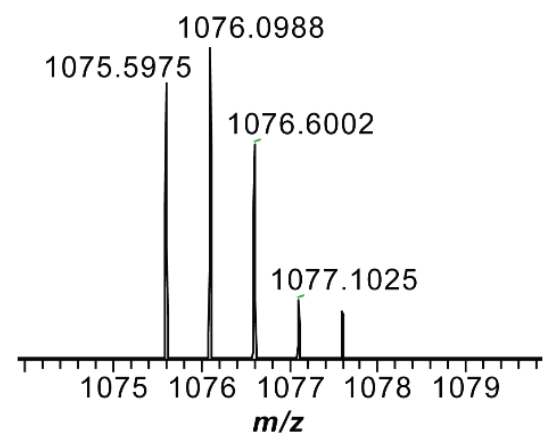

B

\begin{tabular}{|c|c|c|c|}
\hline Ion & $\begin{array}{c}\text { Calculated } \\
\text { mass (Da) }\end{array}$ & $\begin{array}{c}\text { Observed } \\
\text { mass (Da) }\end{array}$ & $\begin{array}{c}\text { Error } \\
\text { (ppm) }\end{array}$ \\
\hline b9 & 999.4974 & 999.4968 & 0.60 \\
\hline b10 & 1112.5814 & 1112.5808 & 0.54 \\
\hline b11 & 1225.6655 & 1225.6644 & 0.90 \\
\hline b12 & 1372.7339 & 1372.7332 & 0.51 \\
\hline b13 & 1471.8023 & 1471.8020 & 0.20 \\
\hline b14 & 1618.8707 & 1618.8706 & 0.06 \\
\hline y4 & 532.3247 & 532.3242 & 0.94 \\
\hline y5 & 679.3932 & 679.3926 & 0.88 \\
\hline y6 & 778.4616 & 778.4611 & 0.64 \\
\hline y7 & 925.5300 & 925.5293 & 0.76 \\
\hline y8 & 1038.6140 & 1038.6136 & 0.39 \\
\hline y9 & 1151.6981 & 1151.6979 & 0.17 \\
\hline
\end{tabular}

C

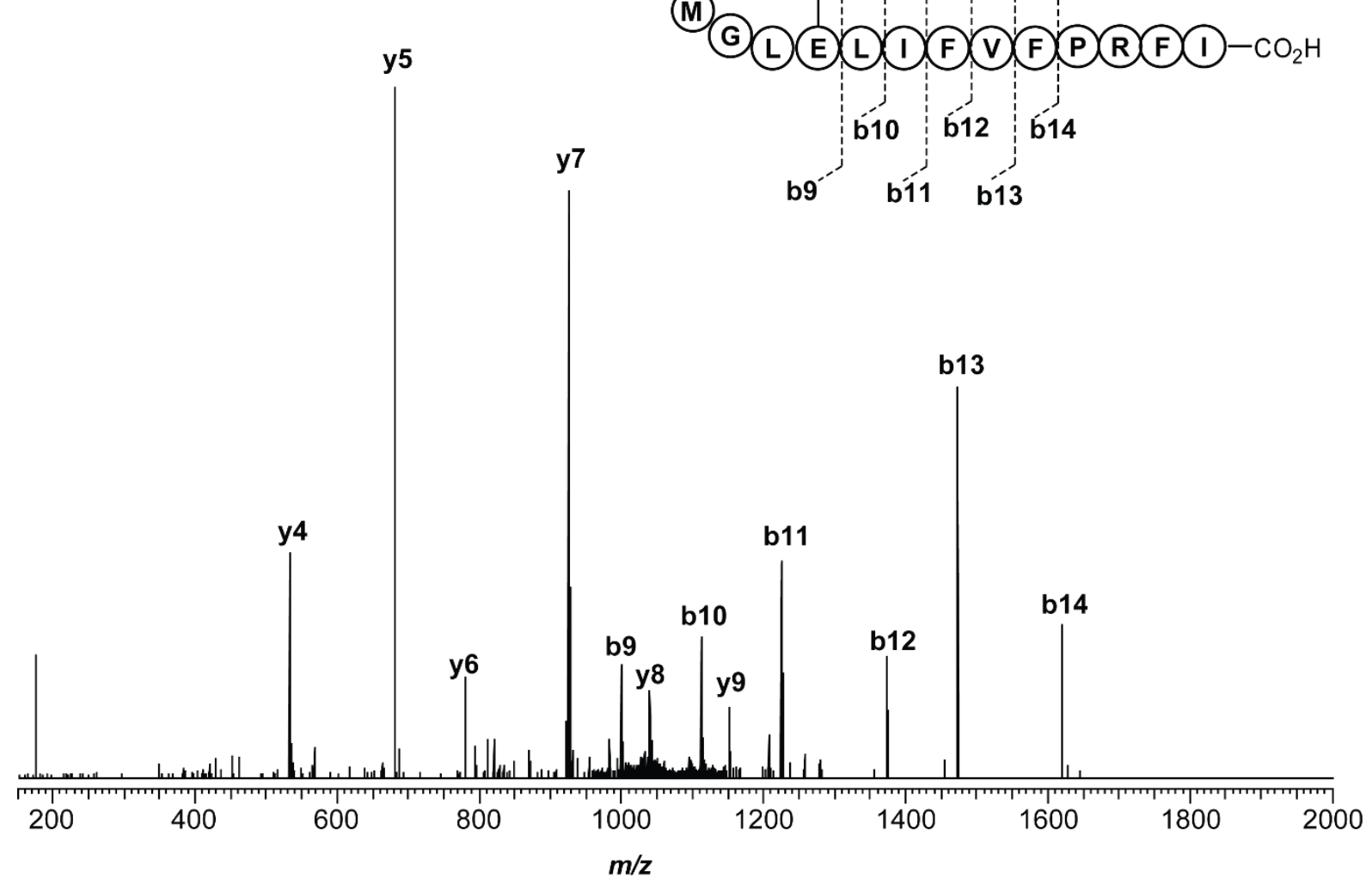

Figure S23. High-resolution and tandem MS of LSSLM variant produced from CFB. (A) High-resolution broadband spectrum of fusilassin LSSLM variant, produced from CFB. (B) Mass assignment for the $\mathrm{b}^{+}$and $\mathrm{y}^{+}$ions generated from CID of fusilassin LSSLM variant. (C) Tandem MS spectrum of fusilassin LSSLM variant, consistent with a Trp1-Glu9 macrolactam linkage. 
A

Formula: $\mathrm{C}_{111} \mathrm{H}_{158} \mathrm{~N}_{22} \mathrm{O}_{24}$

Cal. $[\mathrm{M}+2 \mathrm{H}]^{2+}: 1092.5982 \mathrm{Da}$

Obsv. $[\mathrm{M}+2 \mathrm{H}]^{2+}: 1092.5985 \mathrm{Da}$

Error: $0.27 \mathrm{ppm}$

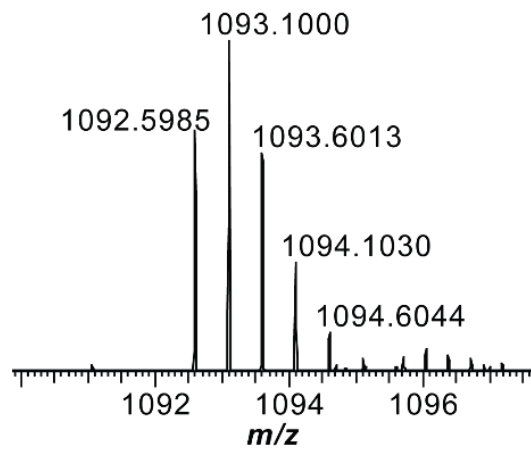

B

\begin{tabular}{|c|c|c|c|}
\hline Ion & $\begin{array}{c}\text { Calculated } \\
\text { mass (Da) }\end{array}$ & $\begin{array}{c}\text { Observed } \\
\text { mass (Da) }\end{array}$ & $\begin{array}{c}\text { Error } \\
\text { (ppm) }\end{array}$ \\
\hline b9 & 1033.4995 & 1033.4989 & 0.58 \\
\hline b10 & 1146.5835 & 1146.5828 & 0.61 \\
\hline b11 & 1259.6676 & 1259.6667 & 0.71 \\
\hline b12 & 1406.7360 & 1406.7357 & 0.21 \\
\hline b13 & 1505.8044 & 1505.8044 & 0 \\
\hline b14 & 1652.8728 & 1652.8719 & 0.54 \\
\hline y4 & 532.3247 & 532.3243 & 0.75 \\
\hline y5 & 679.3932 & 679.3926 & 0.88 \\
\hline y6 & 778.4616 & 778.4611 & 0.64 \\
\hline y7 & 925.5300 & 925.5294 & 0.65 \\
\hline y8 & 1038.6140 & 1038.6133 & 0.67 \\
\hline y9 & 1151.6981 & 1151.6974 & 0.61 \\
\hline
\end{tabular}

C
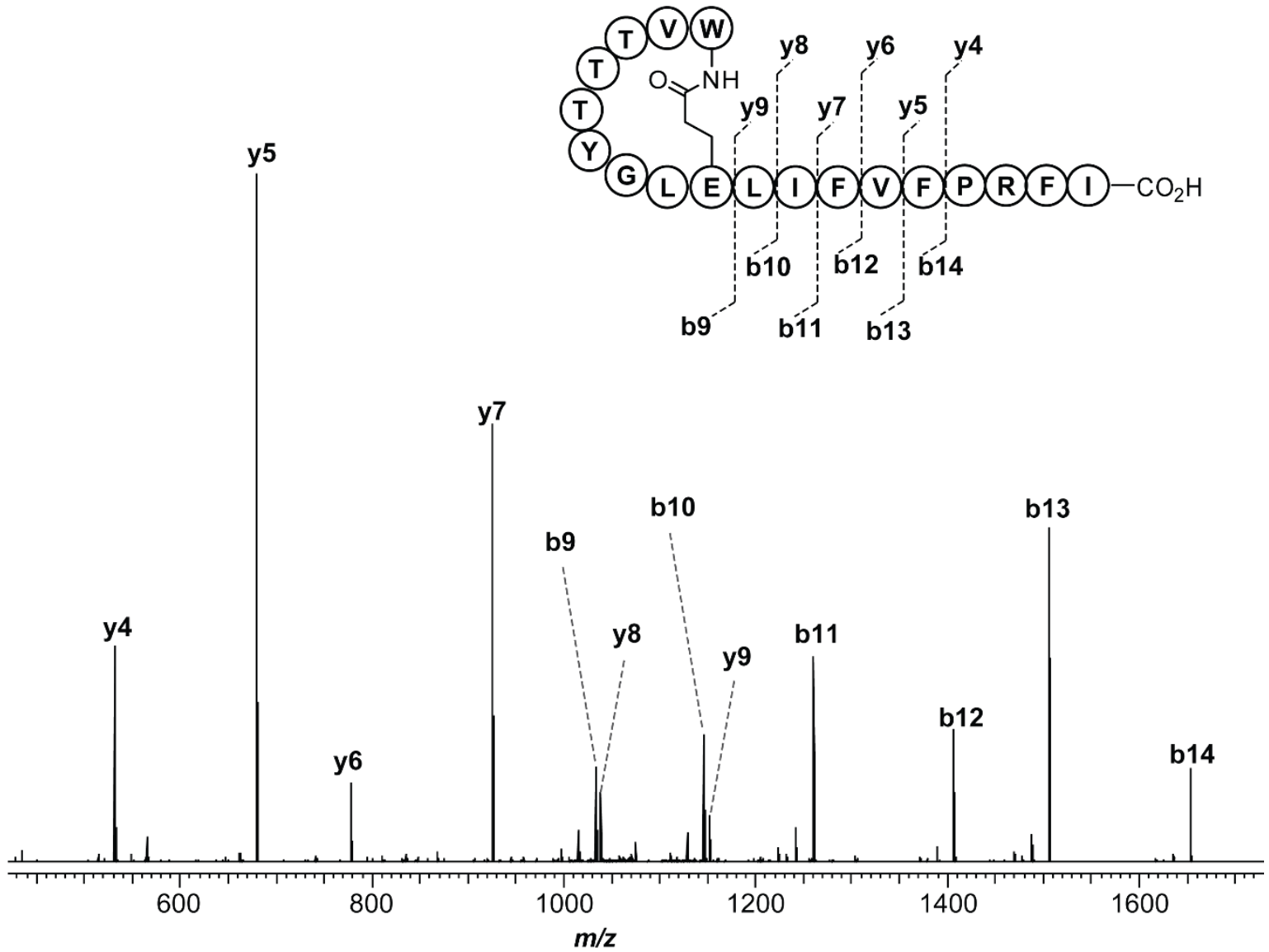

Figure S24. High-resolution and tandem MS of VTTTY variant produced from CFB. (A) High-resolution broadband spectrum of fusilassin VTTTY variant, produced from CFB. (B) Mass assignment for the $\mathrm{b}^{+}$and $\mathrm{y}^{+}$ions generated from CID of fusilassin VTTTY variant. (C) Tandem MS spectrum of fusilassin VTTTY variant, consistent with a Trp1-Glu9 macrolactam linkage. 
A

Formula: $\mathrm{C}_{112} \mathrm{H}_{159} \mathrm{~N}_{23} \mathrm{O}_{23}$

Cal. $[\mathrm{M}+2 \mathrm{H}]^{2+}: 1098.1062 \mathrm{Da}$

Obsv. $[\mathrm{M}+2 \mathrm{H}]^{2+}: 1098.1055 \mathrm{Da}$

Error: 0.64 ppm

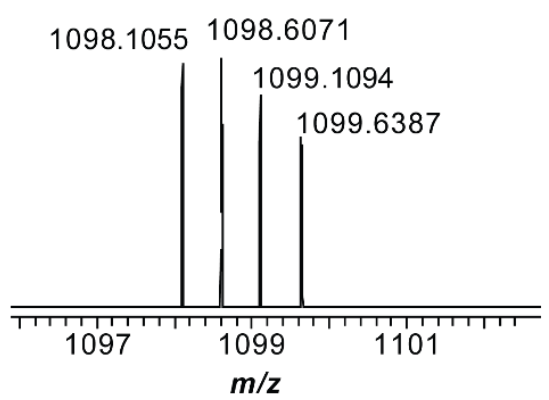

B

\begin{tabular}{|c|c|c|c|}
\hline Ion & $\begin{array}{c}\text { Calculated } \\
\text { mass (Da) }\end{array}$ & $\begin{array}{c}\text { Observed } \\
\text { mass (Da) }\end{array}$ & $\begin{array}{c}\text { Error } \\
\text { (ppm) }\end{array}$ \\
\hline b9 & 1044.5155 & 1044.5135 & 1.91 \\
\hline b10 & 1157.5995 & 1157.5972 & 1.99 \\
\hline b11 & 1270.6836 & 1270.6809 & 1.97 \\
\hline b12 & 1417.7520 & 1417.7499 & 1.48 \\
\hline b13 & 1516.8204 & 1516.8185 & 1.25 \\
\hline b14 & 1663.8888 & 1663.8861 & 1.62 \\
\hline y4 & 532.3247 & 532.3237 & 1.88 \\
\hline y5 & 679.3932 & 679.3918 & 2.06 \\
\hline y6 & 778.4616 & 778.4602 & 1.80 \\
\hline$y 7$ & 925.5300 & 925.5281 & 2.05 \\
\hline y8 & 1038.6140 & 1038.6123 & 1.64 \\
\hline y9 & 1151.6981 & 1151.6963 & 1.56 \\
\hline
\end{tabular}

C
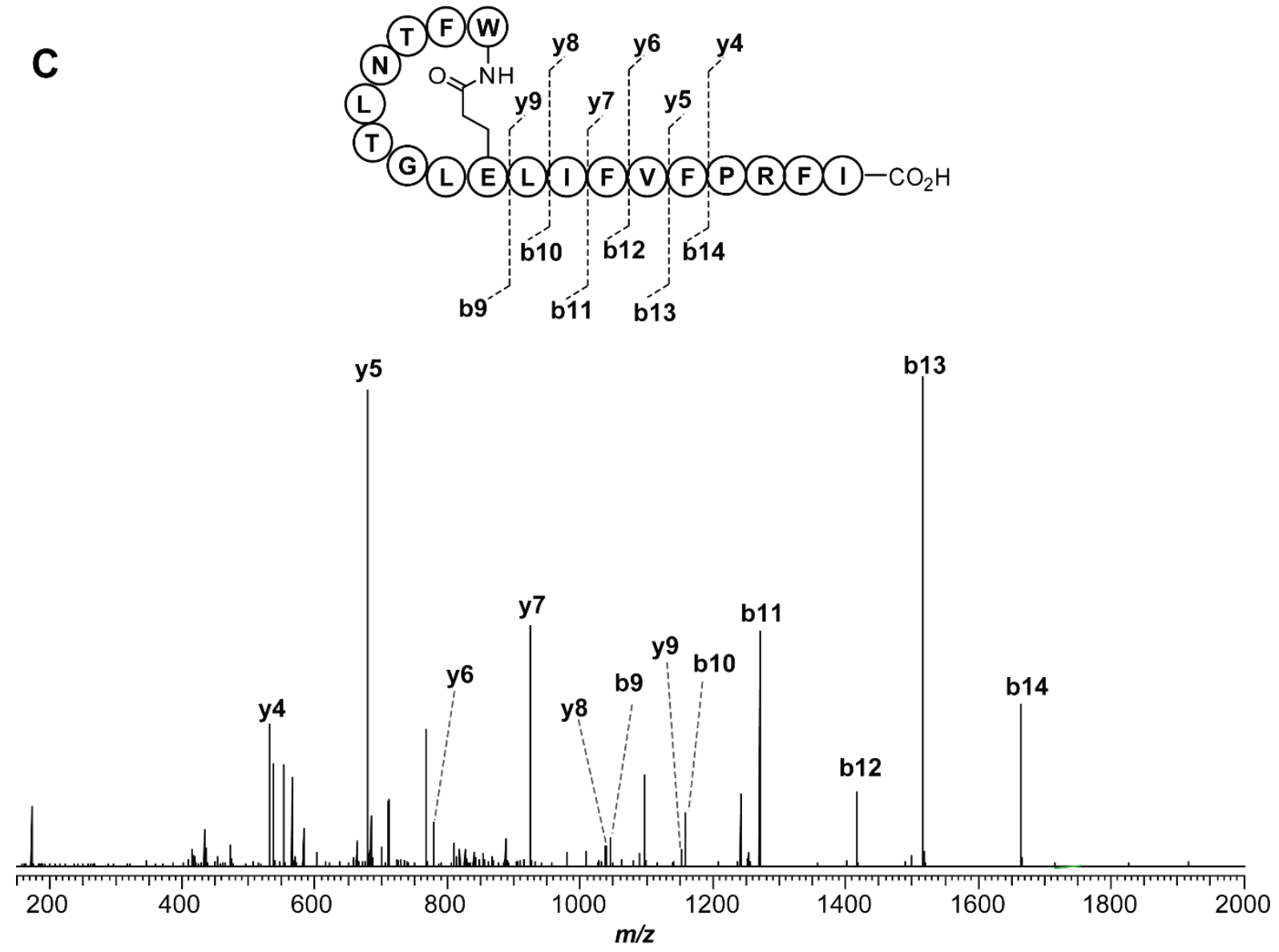

Figure S25. High-resolution and tandem MS of FTNLT variant produced from CFB. (A) High-resolution broadband spectrum of fusilassin FTNLT variant, produced from CFB. (B) Mass assignment for the $\mathrm{b}^{+}$and $\mathrm{y}^{+}$ions generated from CID of fusilassin FTNLT variant. (C) Tandem MS spectrum of fusilassin FTNLT variant, consistent with a Trp1-Glu9 macrolactam linkage. 
A

Formula: $\mathrm{C}_{112} \mathrm{H}_{156} \mathrm{~N}_{24} \mathrm{O}_{23}$

Cal. $[\mathrm{M}+2 \mathrm{H}]^{2+}: 1103.5960 \mathrm{Da}$

Obsv. $[\mathrm{M}+2 \mathrm{H}]^{2+}: 1103.5962 \mathrm{Da}$

Error: $0.18 \mathrm{ppm}$

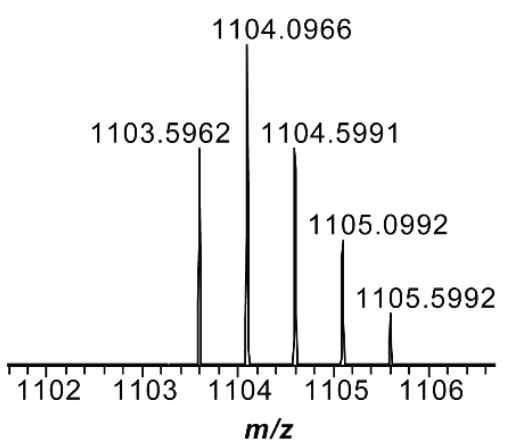

B

\begin{tabular}{|c|c|c|c|}
\hline Ion & $\begin{array}{c}\text { Calculated } \\
\text { mass (Da) }\end{array}$ & $\begin{array}{c}\text { Observed } \\
\text { mass (Da) }\end{array}$ & $\begin{array}{c}\text { Error } \\
\text { (ppm) }\end{array}$ \\
\hline b9 & 1055.4951 & 1055.4941 & 0.95 \\
\hline b10 & 1168.5791 & 1168.5781 & 0.86 \\
\hline b11 & 1281.6632 & 1281.6621 & 0.86 \\
\hline b12 & 1428.7316 & 1428.7310 & 0.42 \\
\hline b13 & 1527.8000 & 1527.7998 & 0.13 \\
\hline b14 & 1674.8684 & 1674.8674 & 0.60 \\
\hline y4 & 532.3247 & 532.3242 & 0.94 \\
\hline y5 & 679.3932 & 679.3925 & 1.03 \\
\hline y6 & 778.4616 & 778.4609 & 0.90 \\
\hline y7 & 925.5300 & 925.5292 & 0.86 \\
\hline y8 & 1038.6140 & 1038.6128 & 1.16 \\
\hline y9 & 1151.6981 & 1151.6979 & 0.17 \\
\hline
\end{tabular}

C

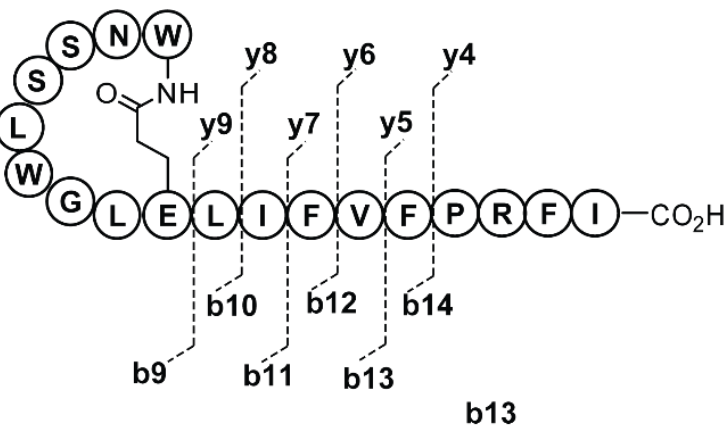

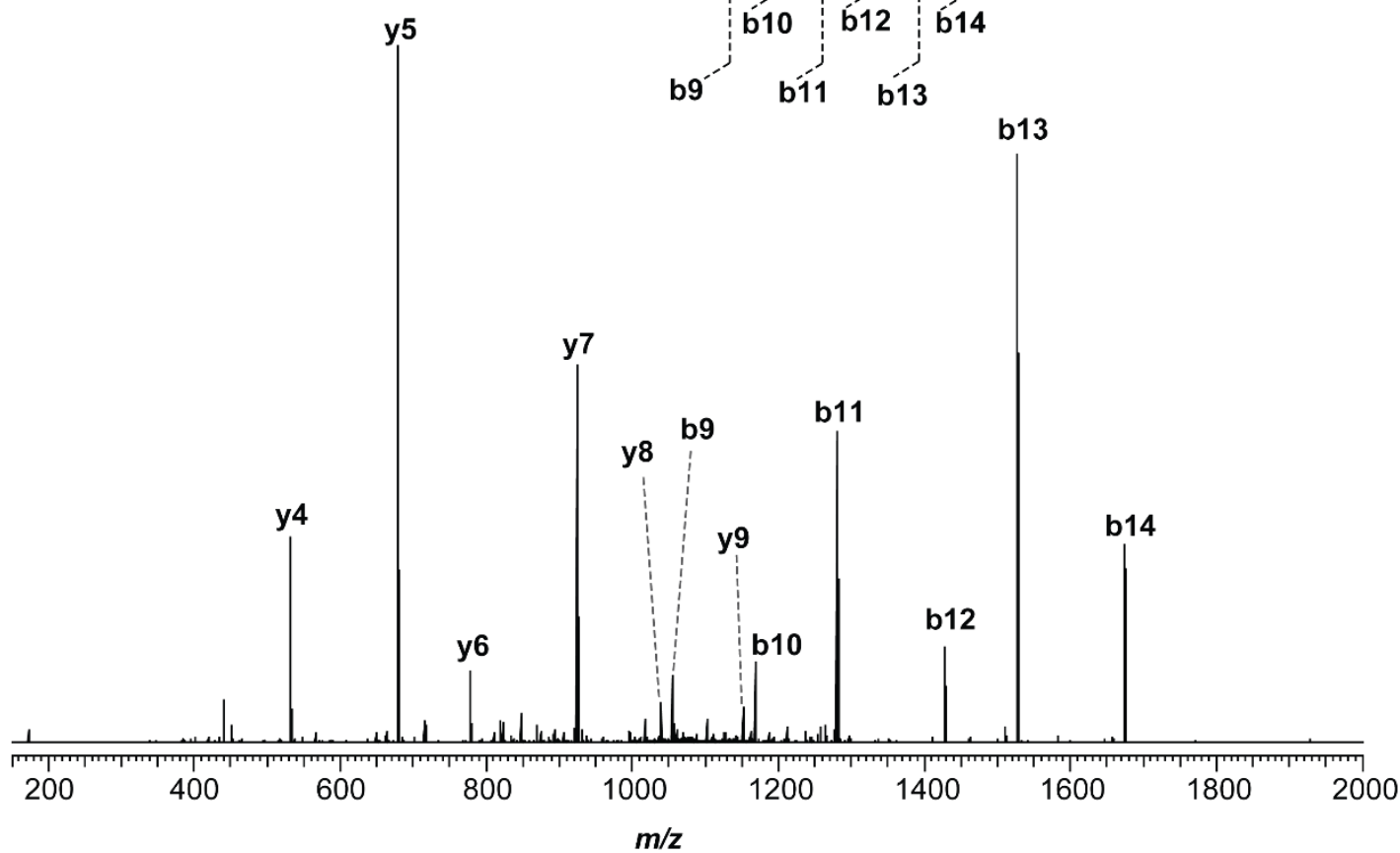

Figure S26. High-resolution and tandem MS of NSSLW variant produced from CFB. (A) High-resolution broadband spectrum of fusilassin NSSLW variant, produced from CFB. (B) Mass assignment for the $\mathrm{b}^{+}$and $\mathrm{y}^{+}$ions generated from CID of fusilassin NSSLW variant. (C) Tandem MS spectrum of fusilassin NSSLW variant, consistent with a Trp1-Glu9 macrolactam linkage. 
A

Formula: $\mathrm{C}_{117} \mathrm{H}_{158} \mathrm{~N}_{22} \mathrm{O}_{22} \mathrm{~S}$

Cal. $[\mathrm{M}+2 \mathrm{H}]^{2+}: 1116.5893 \mathrm{Da}$

Obsv. $[\mathrm{M}+2 \mathrm{H}]^{2+}: 1116.5886 \mathrm{Da}$

Error: $0.63 \mathrm{ppm}$

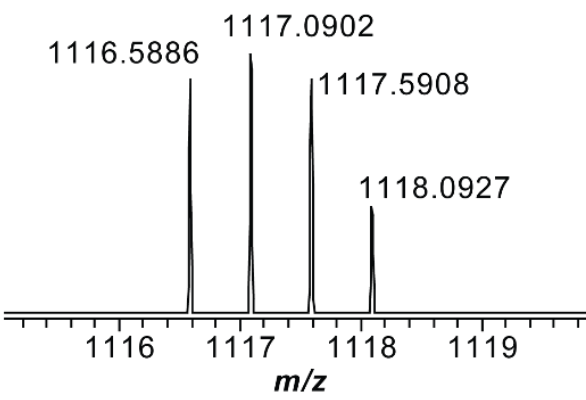

B

\begin{tabular}{|c|c|c|c|}
\hline Ion & $\begin{array}{c}\text { Calculated } \\
\text { mass (Da) }\end{array}$ & $\begin{array}{c}\text { Observed } \\
\text { mass (Da) }\end{array}$ & $\begin{array}{c}\text { Error } \\
\text { (ppm) }\end{array}$ \\
\hline b9 & 1081.4817 & 1081.4791 & 2.40 \\
\hline b10 & 1194.5658 & 1194.5634 & 2.01 \\
\hline b11 & 1307.6498 & 1307.6473 & 1.91 \\
\hline b12 & 1454.7183 & 1454.7156 & 1.86 \\
\hline b13 & 1553.7867 & 1553.7845 & 1.42 \\
\hline b14 & 1700.8551 & 1700.8512 & 2.29 \\
\hline y4 & 532.3247 & 532.3237 & 1.88 \\
\hline y5 & 679.3932 & 679.3918 & 2.06 \\
\hline y6 & 778.4616 & 778.4606 & 1.28 \\
\hline y7 & 925.5300 & 925.5281 & 2.05 \\
\hline
\end{tabular}

C
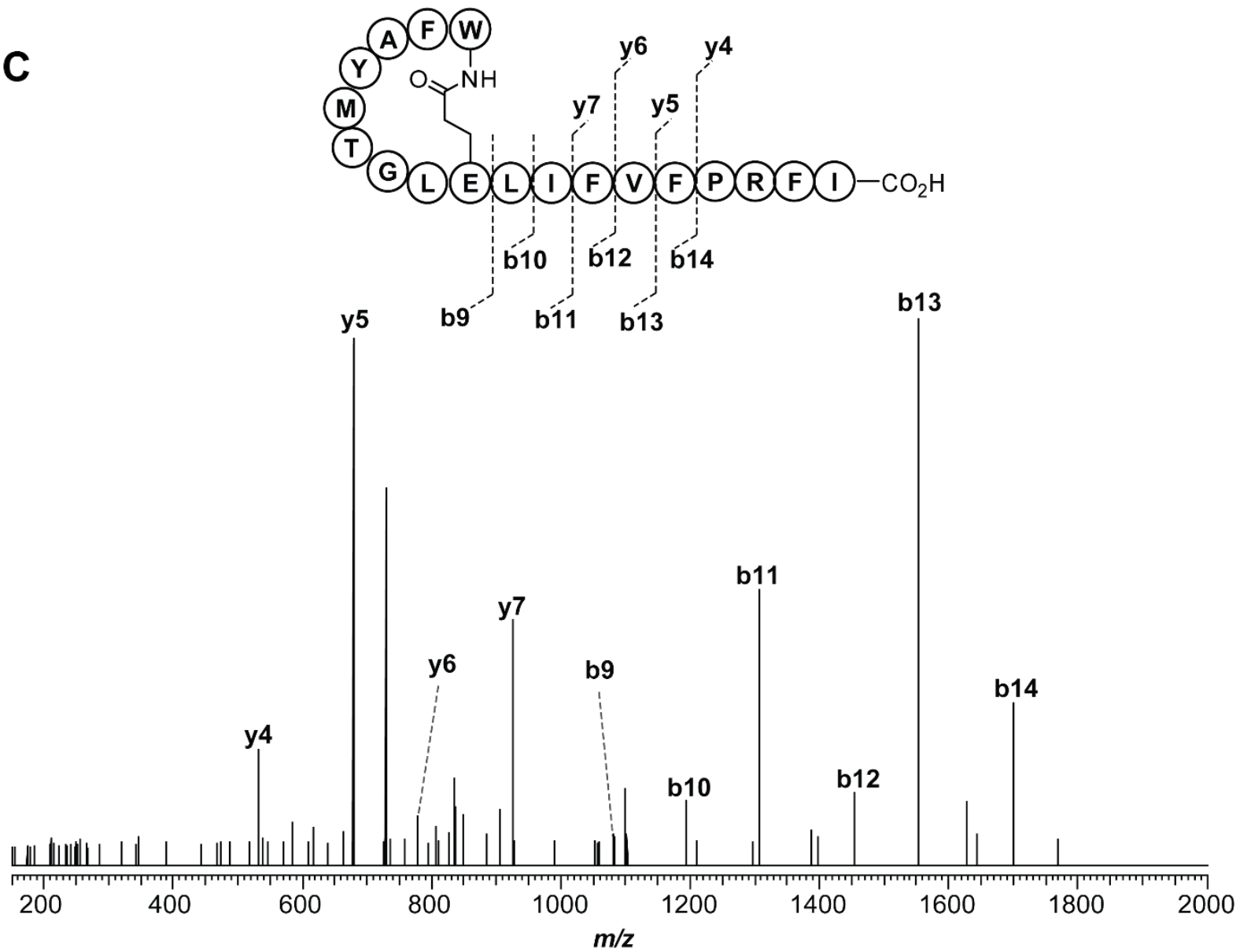

Figure S27. High-resolution and tandem MS of FAYMT variant produced from CFB. (A) High-resolution broadband spectrum of fusilassin FAYMT variant, produced from CFB. (B) Mass assignment for the $\mathrm{b}^{+}$and $\mathrm{y}^{+}$ions generated from CID of fusilassin FAYMT variant. (C) Tandem MS spectrum of fusilassin FAYMT variant, consistent with a Trp1-Glu9 macrolactam linkage. 
A

Formula: $\mathrm{C}_{119} \mathrm{H}_{161} \mathrm{~N}_{25} \mathrm{O}_{21}$

Cal. $[\mathrm{M}+2 \mathrm{H}]^{2+}: 1139.1222 \mathrm{Da}$

Obsv. $[\mathrm{M}+2 \mathrm{H}]^{2+}: 1139.1207 \mathrm{Da}$

Error: $1.32 \mathrm{ppm}$

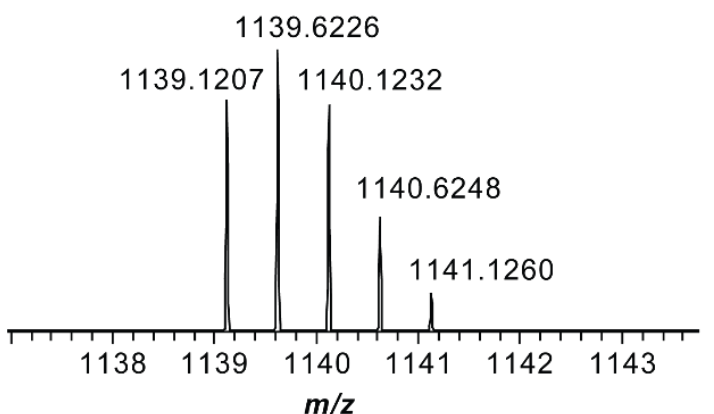

B

\begin{tabular}{|c|c|c|c|}
\hline Ion & $\begin{array}{c}\text { Calculated } \\
\text { mass (Da) }\end{array}$ & $\begin{array}{c}\text { Observed } \\
\text { mass (Da) }\end{array}$ & $\begin{array}{c}\text { Error } \\
\text { (ppm) }\end{array}$ \\
\hline b9 & 1126.5474 & 1126.5454 & 1.78 \\
\hline b10 & 1239.6315 & 1239.6294 & 1.69 \\
\hline b11 & 1352.7156 & 1352.7134 & 1.63 \\
\hline b12 & 1499.7840 & 1499.7825 & 1.00 \\
\hline b13 & 1598.8524 & 1598.8503 & 1.31 \\
\hline b14 & 1745.9208 & 1745.9175 & 1.89 \\
\hline y4 & 532.3247 & 532.3237 & 1.88 \\
\hline y5 & 679.3932 & 679.3920 & 1.77 \\
\hline y6 & 778.4616 & 778.4602 & 1.80 \\
\hline y7 & 925.5300 & 925.5283 & 1.84 \\
\hline y8 & 1038.6140 & 1038.6121 & 1.83 \\
\hline y9 & 1151.6981 & 1151.6963 & 1.56 \\
\hline
\end{tabular}

C
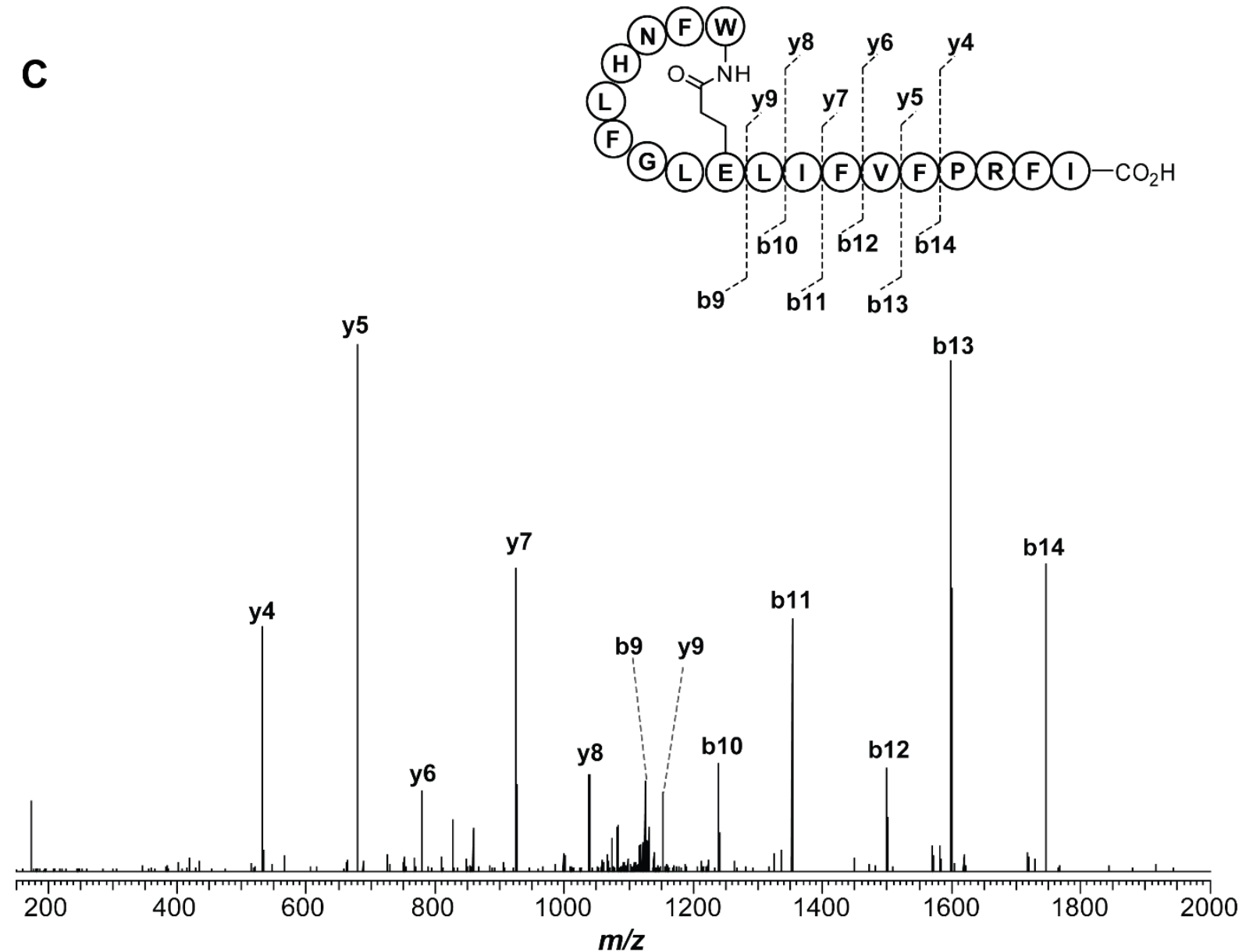

Figure S28. High-resolution and tandem MS of FNHLF variant produced from CFB. (A) High-resolution broadband spectrum of fusilassin FNHLF variant, produced from CFB. (B) Mass assignment for the $\mathrm{b}^{+}$and $\mathrm{y}^{+}$ions generated from CID of fusilassin FNHLF variant. (C) Tandem MS spectrum of fusilassin FNHLF variant, consistent with a Trp1-Glu9 macrolactam linkage. 
A

Formula: $\mathrm{C}_{120} \mathrm{H}_{159} \mathrm{~N}_{23} \mathrm{O}_{24}$ Cal. $[\mathrm{M}+2 \mathrm{H}]^{2+}: 1154.1037 \mathrm{Da}$

Obsv. $[\mathrm{M}+2 \mathrm{H}]^{2++}: 1154.1041 \mathrm{Da}$

Error: $0.35 \mathrm{ppm}$

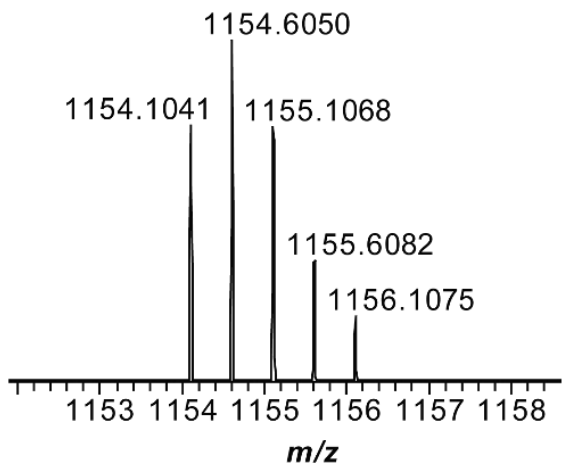

B

\begin{tabular}{|c|c|c|c|}
\hline Ion & $\begin{array}{c}\text { Calculated } \\
\text { mass (Da) }\end{array}$ & $\begin{array}{c}\text { Observed } \\
\text { mass (Da) }\end{array}$ & $\begin{array}{c}\text { Error } \\
\text { (ppm) }\end{array}$ \\
\hline b9 & 1156.5104 & 1156.5093 & 0.78 \\
\hline b10 & 1269.5944 & 1269.5936 & 0.63 \\
\hline b11 & 1382.6785 & 1382.6776 & 0.65 \\
\hline b12 & 1529.7469 & 1529.7467 & 0.13 \\
\hline b13 & 1628.8153 & 1628.8147 & 0.37 \\
\hline b14 & 1775.8837 & 1775.8818 & 1.07 \\
\hline y4 & 532.3247 & 532.3242 & 0.94 \\
\hline y5 & 679.3932 & 679.3926 & 0.88 \\
\hline y6 & 778.4616 & 778.4609 & 0.90 \\
\hline y7 & 925.5300 & 925.5294 & 0.65 \\
\hline y8 & 1038.6140 & 1038.6133 & 0.67 \\
\hline y9 & 1151.6981 & 1151.6976 & 0.43 \\
\hline
\end{tabular}

C
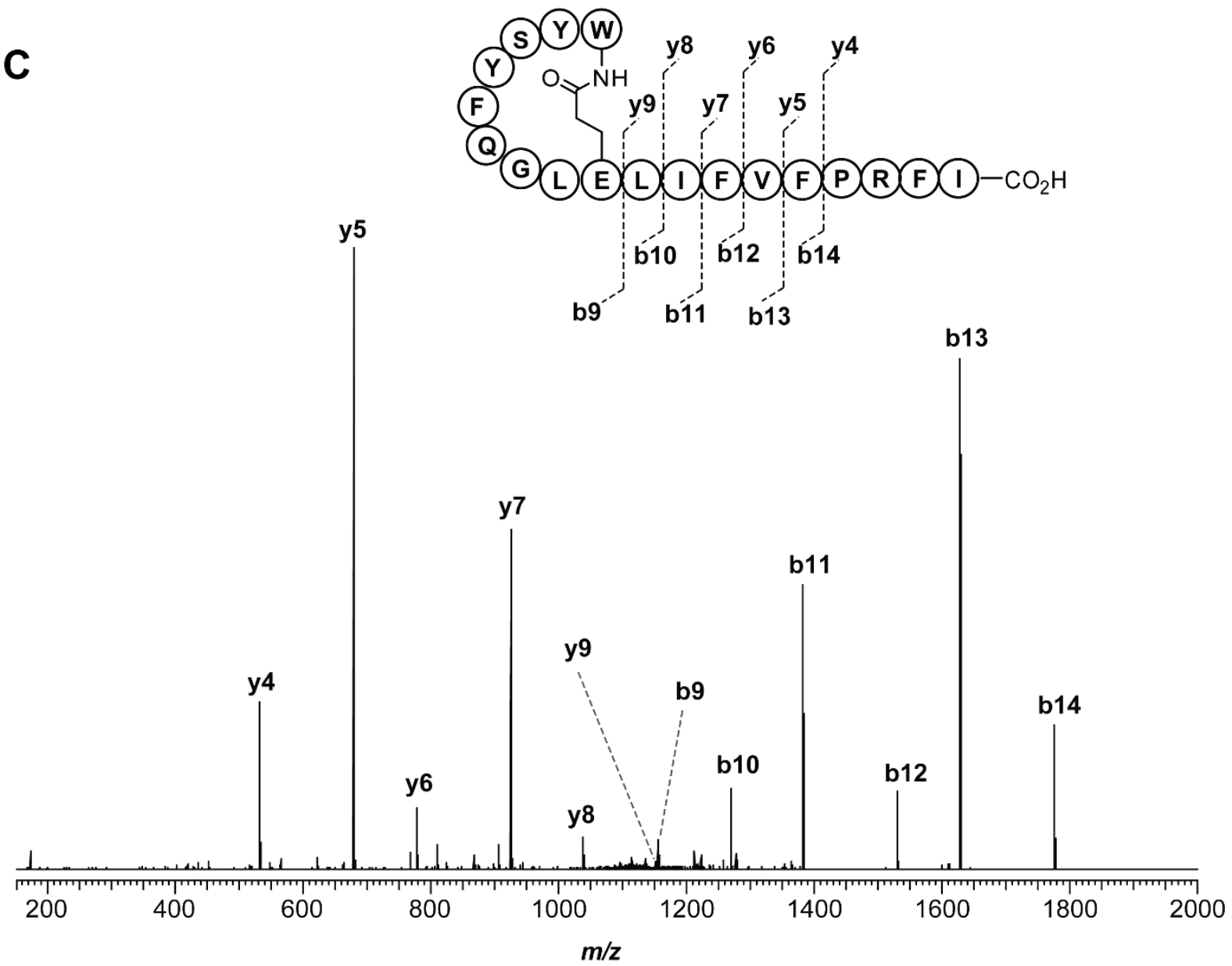

Figure S29. High-resolution and tandem MS of YSYFQ variant produced from CFB. (A) High-resolution broadband spectrum of fusilassin YSYFQ variant, produced from CFB. (B) Mass assignment for the $\mathrm{b}^{+}$and $\mathrm{y}^{+}$ions generated from CID of fusilassin YSYFQ variant. (C) Tandem MS spectrum of fusilassin YSYFQ variant, consistent with a Trp1-Glu9 macrolactam linkage. 
A

WTVQGAGLELIFVFPRFI (m/z 2075)

(1)

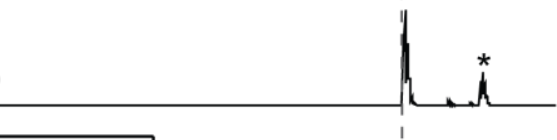

WTVQGAGLELIFVFPRF (m/z,1962)

(2)

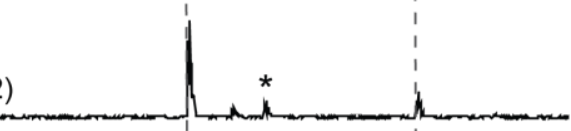

(3)

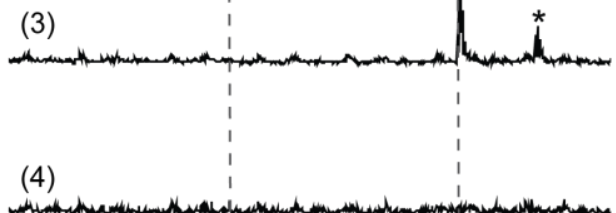

A

19001950200020502100

$m / z$

C

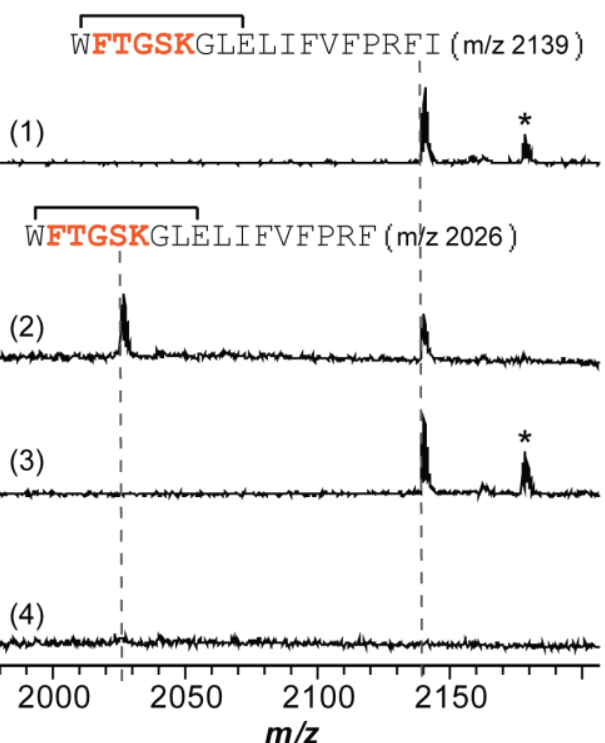

E

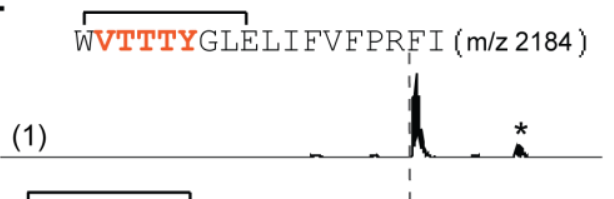
WVTTTYGLELIFVFPRF ( $\mathrm{m} / \mathrm{z} 2071)$

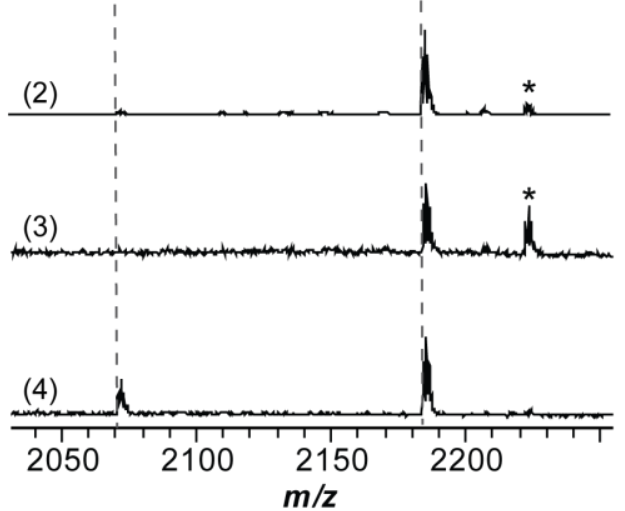

B

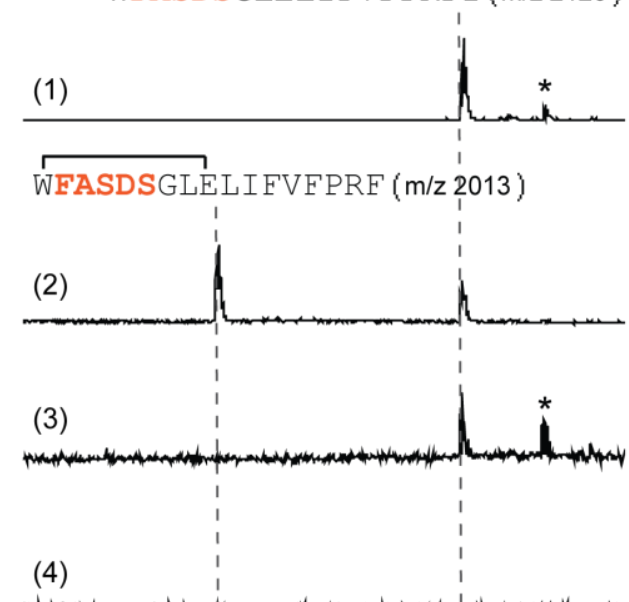

(1) $\mathrm{m} / \mathrm{z}$

D

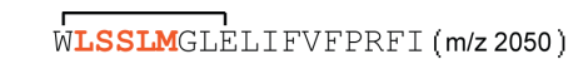

(1)

(2)

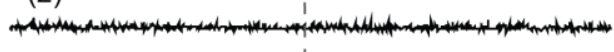

(3)

(4)

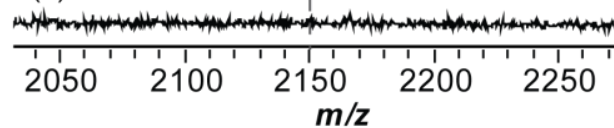

$\mathbf{F}$
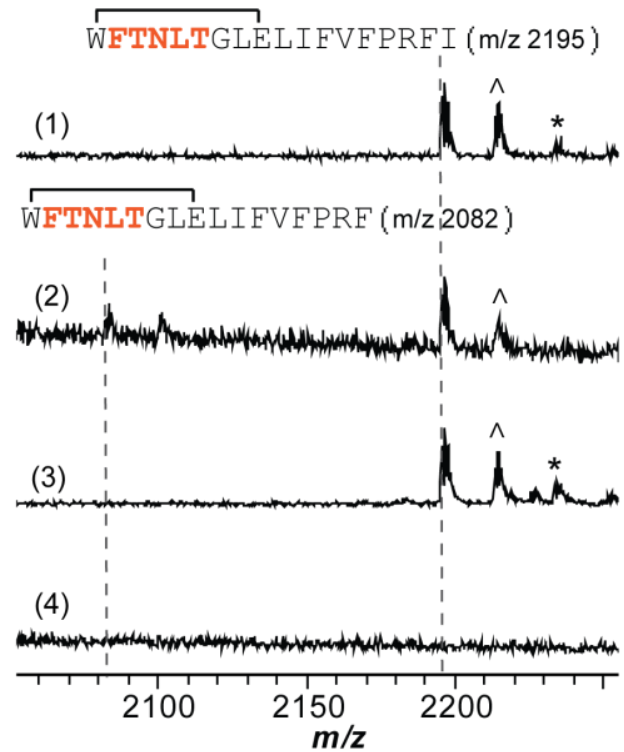

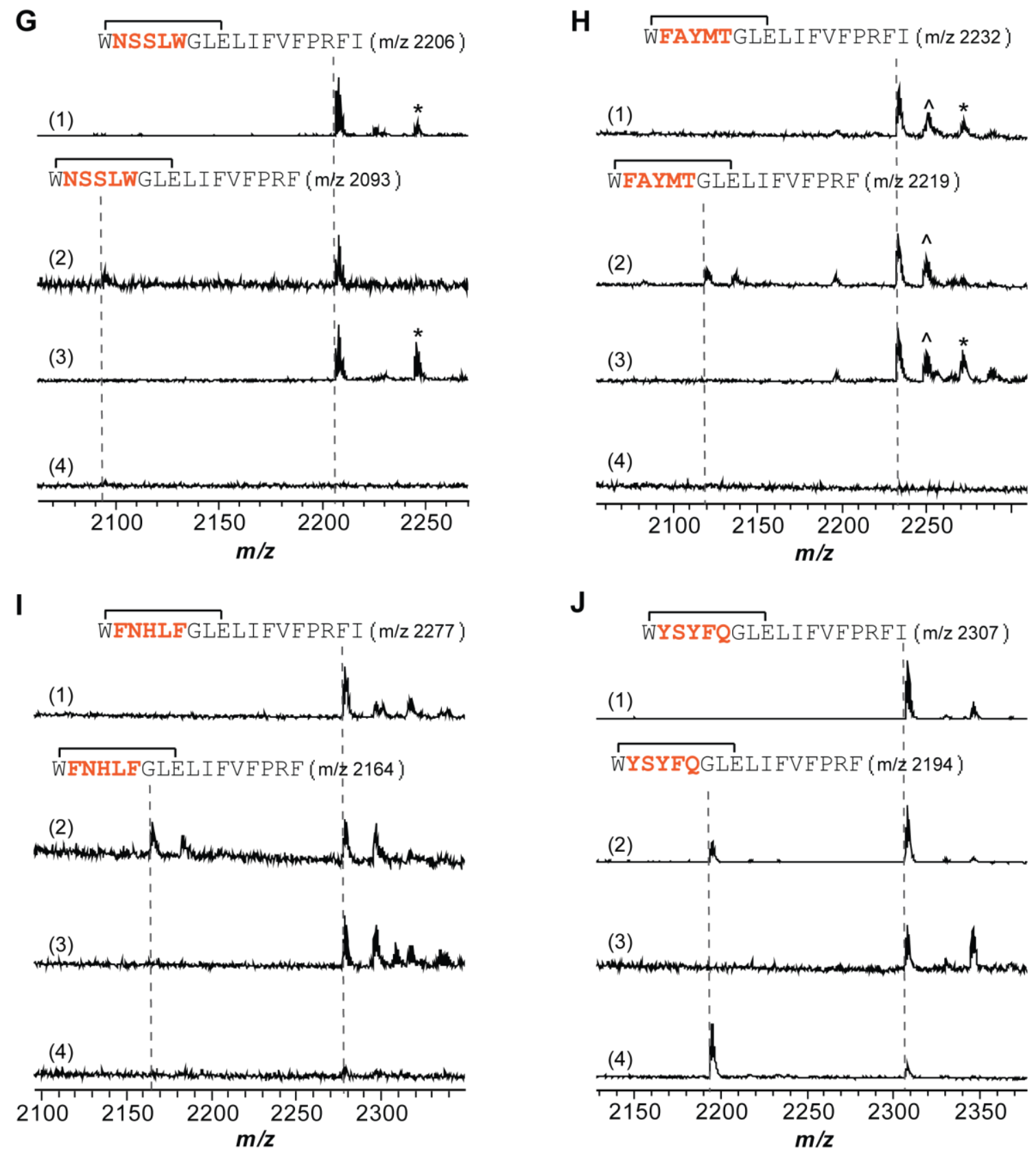

Figure S30. Carboxypeptidase $\mathbf{Y}$ digestion for fusilassin ring variants. FusA ring variants are produced through $\mathrm{CFB}$ (1), and the corresponding lasso peptide was treated under 3 different conditions: carboxypeptidase $\mathrm{Y}$ digestion for $18 \mathrm{~h} \mathrm{(2)}$; heat treatment at $95{ }^{\circ} \mathrm{C}$ for $2 \mathrm{~h}$ in $60 \%$ ACN (3); heat treatment at $95{ }^{\circ} \mathrm{C}$ for $2 \mathrm{~h}$ in $60 \%$ ACN followed by carboxypeptidase $\mathrm{Y}$ digestion for $18 \mathrm{~h}$ at room temperature (4). The sequence of each FusA ring variant is shown in the graph and the variation sites are orange. (A) TVQGA variant; (B) FASDS variant; (C) FTGSK variant; (D) LSSLM variant; (E) VTTTY variant; (F) FTNLT variant; (G) NSSLW variant; (H) FAYMT variant; (I) FNHLF variant; (J) YSYFQ variant. The absence/presence of intact lasso peptide upon treatment under condition (4) and the presence of intact lasso peptide under condition (2) and (3) indicate the threaded isomer of lasso peptide. 


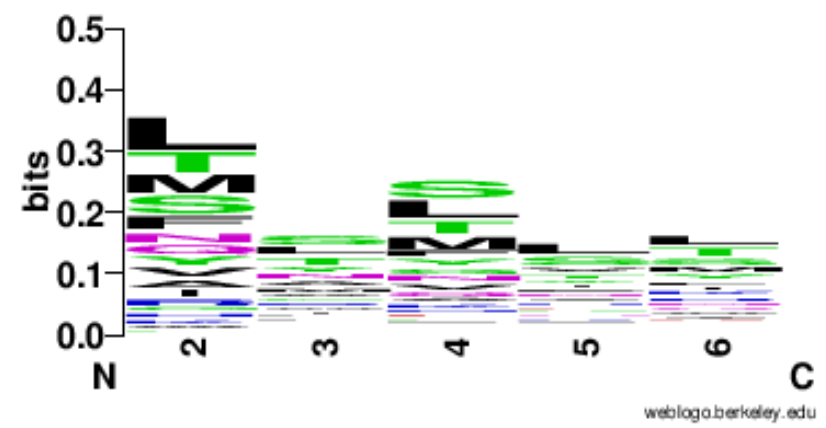

Figure S31. Sequence logo of sequence-confirmed FusC substrates. The logo was created using 280 sequence-confirmed FusC substrates in WebLogo version 2.8.2. ${ }^{19}$ 


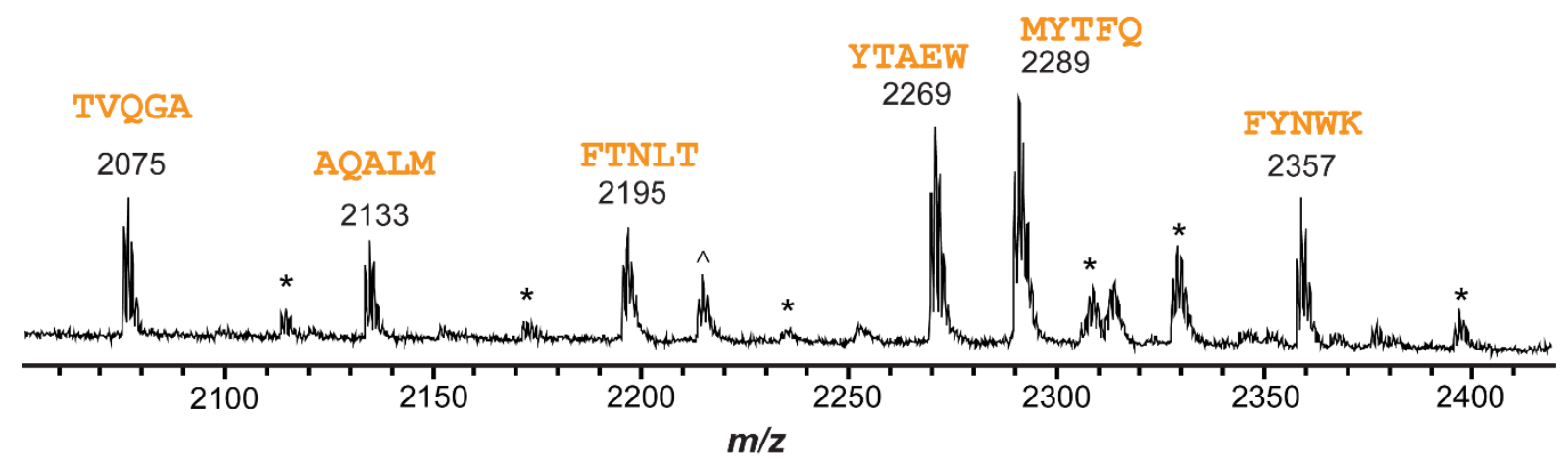

Figure S32. Simultaneous production of multiple fusilassin variants using CFB. MALDI-TOF mass spectrum of six fusilassin ring variants simultaneously produced in a single CFB reaction. The sequences of the varied region (residues 2-6) are highlighted in orange. The mass label corresponds to the $[\mathrm{M}+\mathrm{H}]^{+}$ion of the lasso peptide. ${ }^{*}$ indicates the $[\mathrm{M}+\mathrm{K}]^{+}$ion for the lasso peptide. ${ }^{\wedge}$ indicates the uncyclized linear core peptide. 


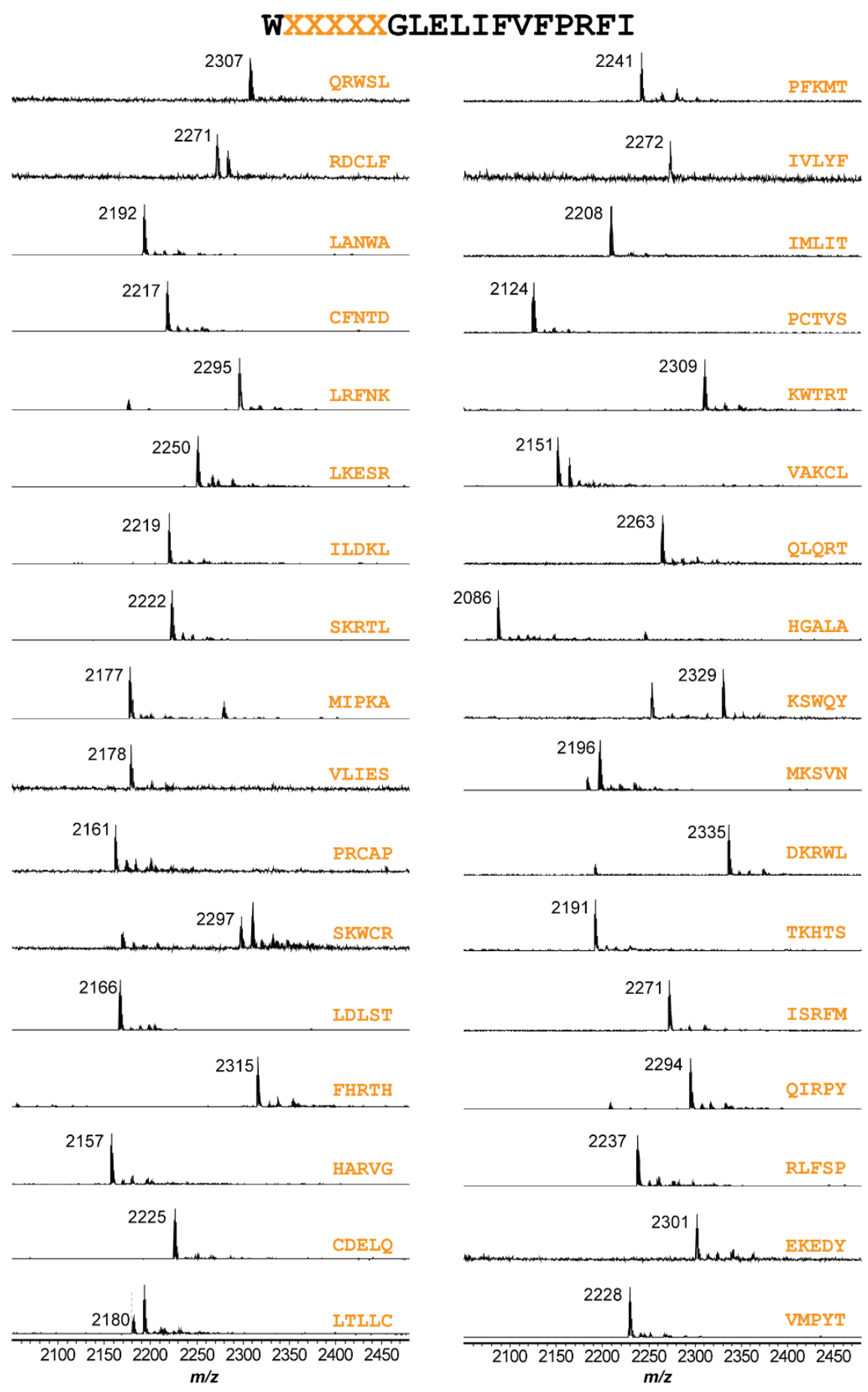

Figure S33. MALDI-TOF-MS spectra of the uncyclized linear core peptides of the FusC nonsubstrates produced from PURExpress. 154 FusC nonsubstrates that showed no signal from CFB were sequenced, and their corresponding linear core peptides were detected by MALDI-TOF-MS from the PURExpress reaction, in which purified FusB, C, and E were added, indicating that FusB/E was able to cleave the leader peptide, while FusC failed to cyclize the cleaved core. Orange depicts the varied sequence of positions 2-6 of the FusA core region. The mass label corresponds to the $[\mathrm{M}+\mathrm{H}]^{+}$ion of the uncyclized linear core peptide. Figure continues onto the next 2 pages. 
WXXXXXGLELIFVFPRFI

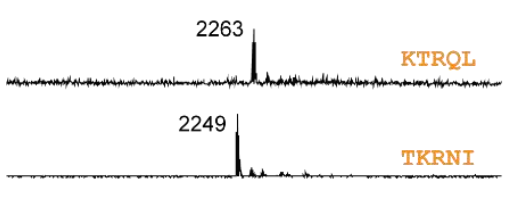

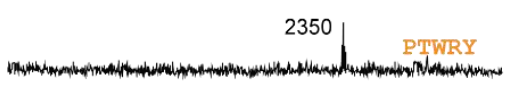
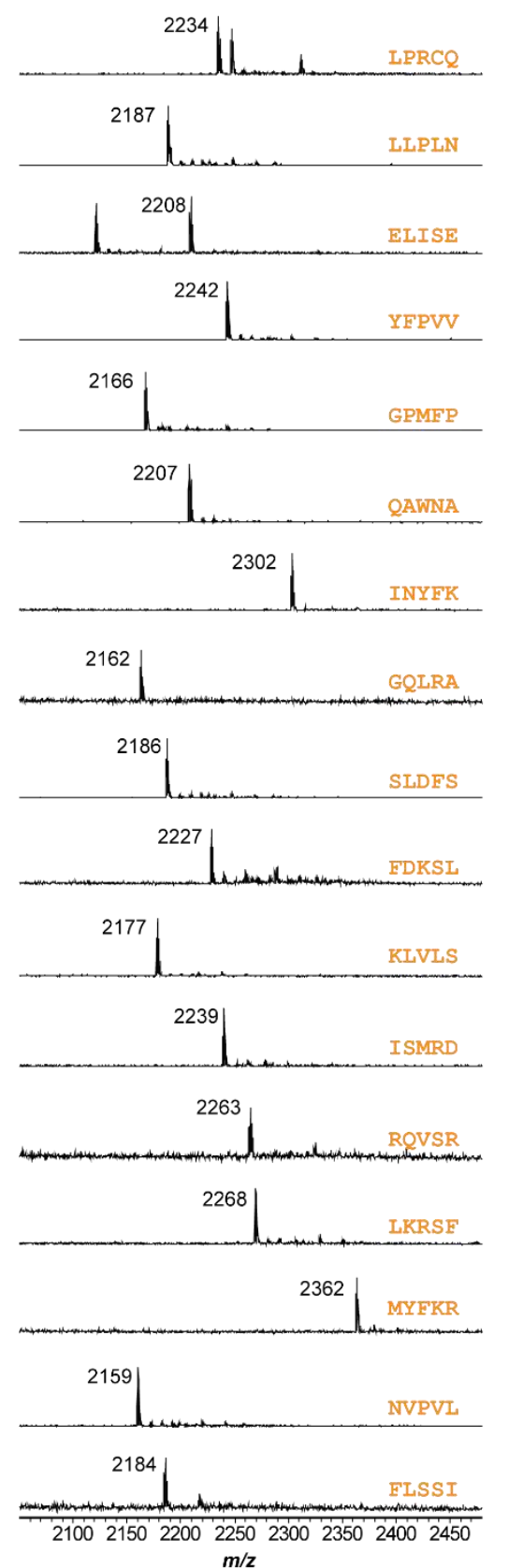
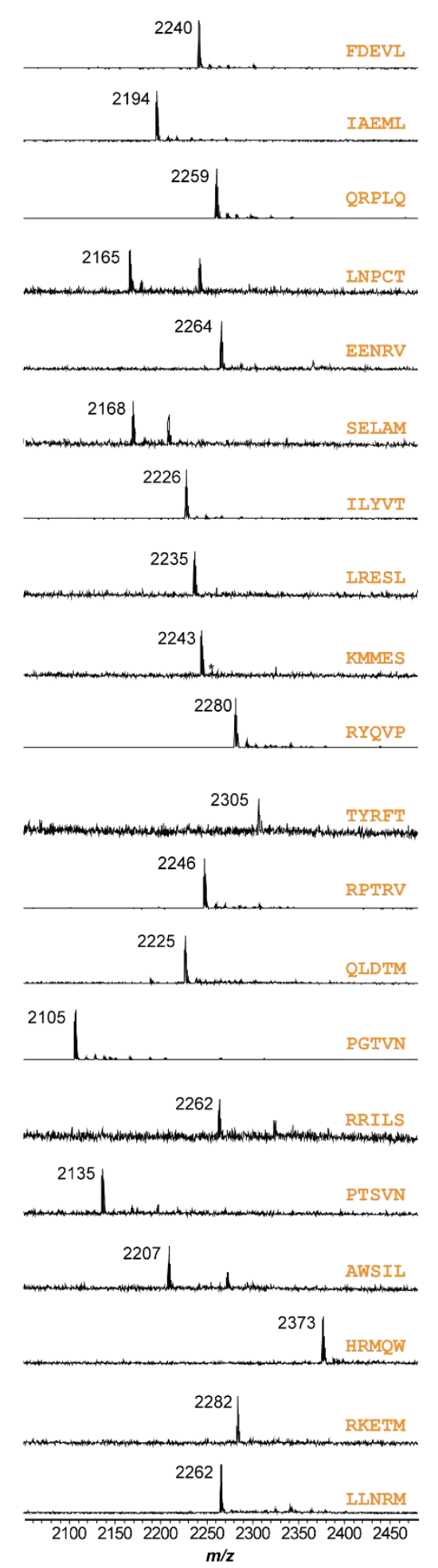
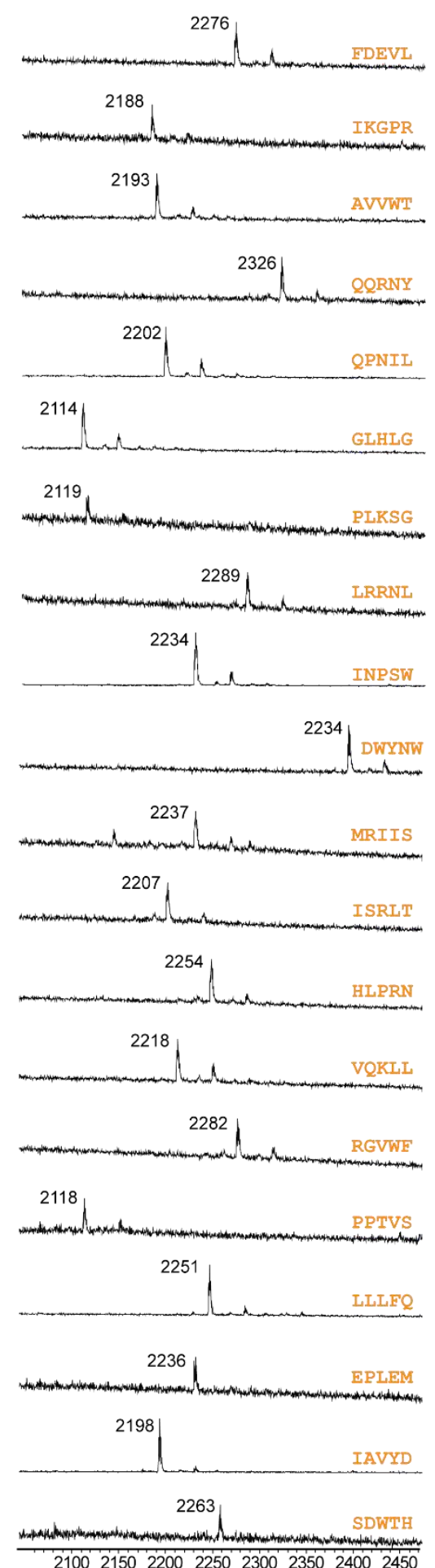

21002150220022502300235024002450 $\mathrm{m} / \mathbf{z}$

Figure S33. Continued from previous page (2 of 3) 
WXXXXXGLELIFVFPRFI
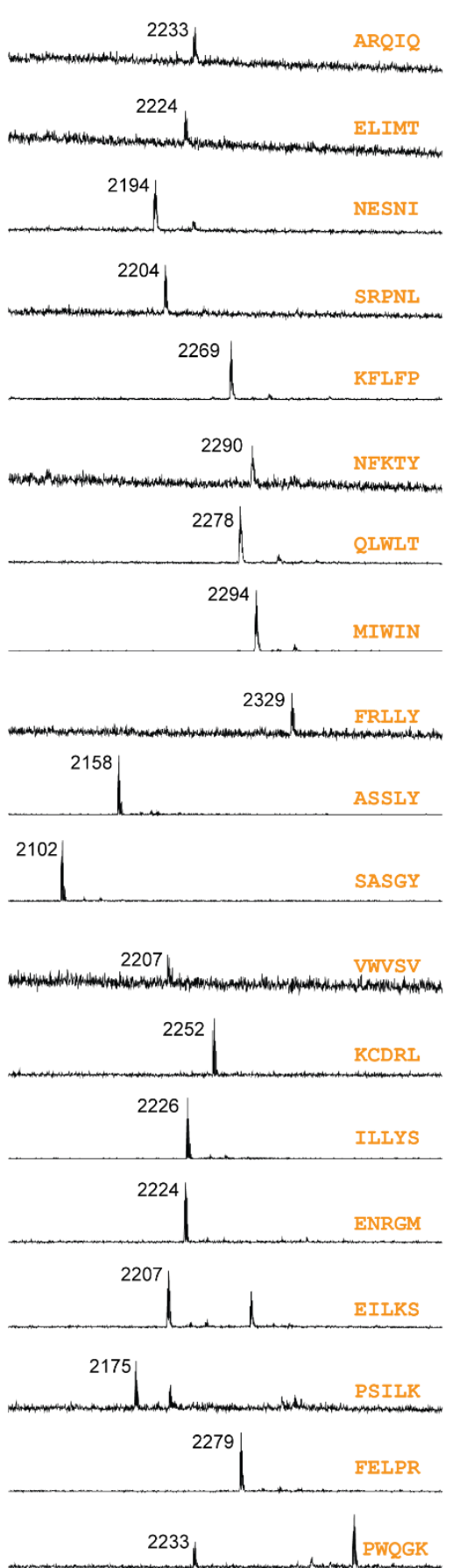

${ }^{2237}{ }^{22} \mid$ MDIIO

$2100 \quad 21502200 \quad 22502300 \quad 235024002450$ $m / z$
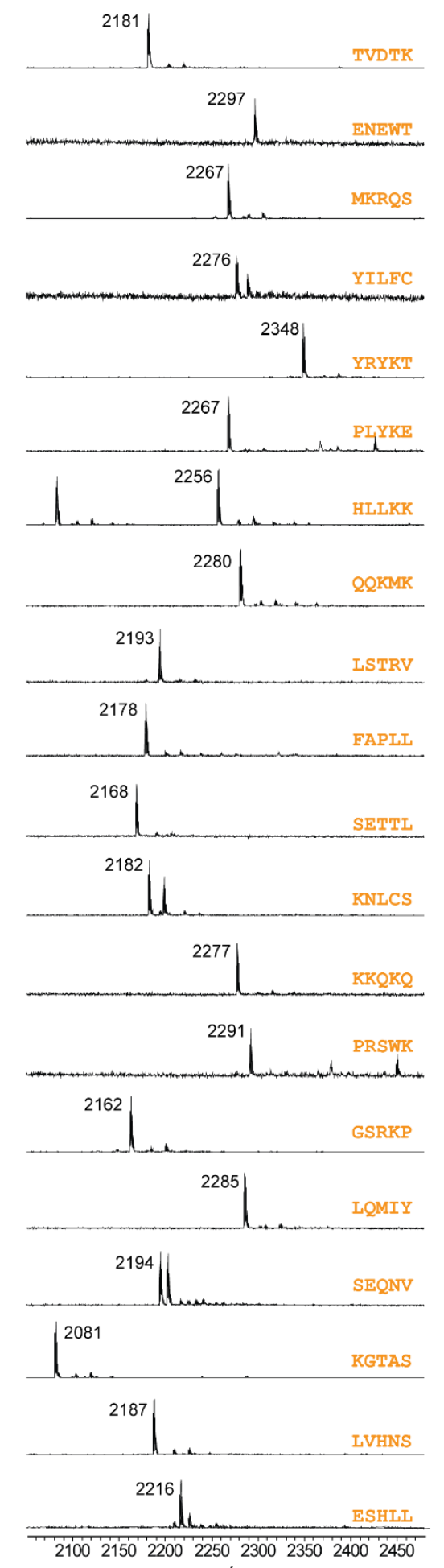
$m / z$
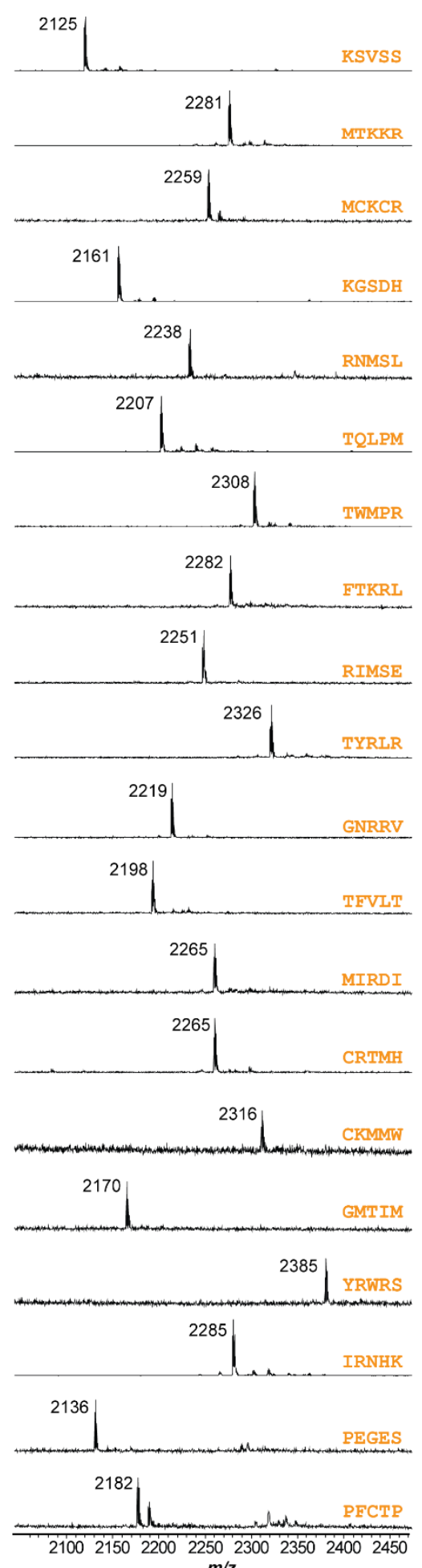

Figure S33. Continued from previous page ( 3 of 3 ) 


\section{WXXXXXGLELIFVFPRFI}

A

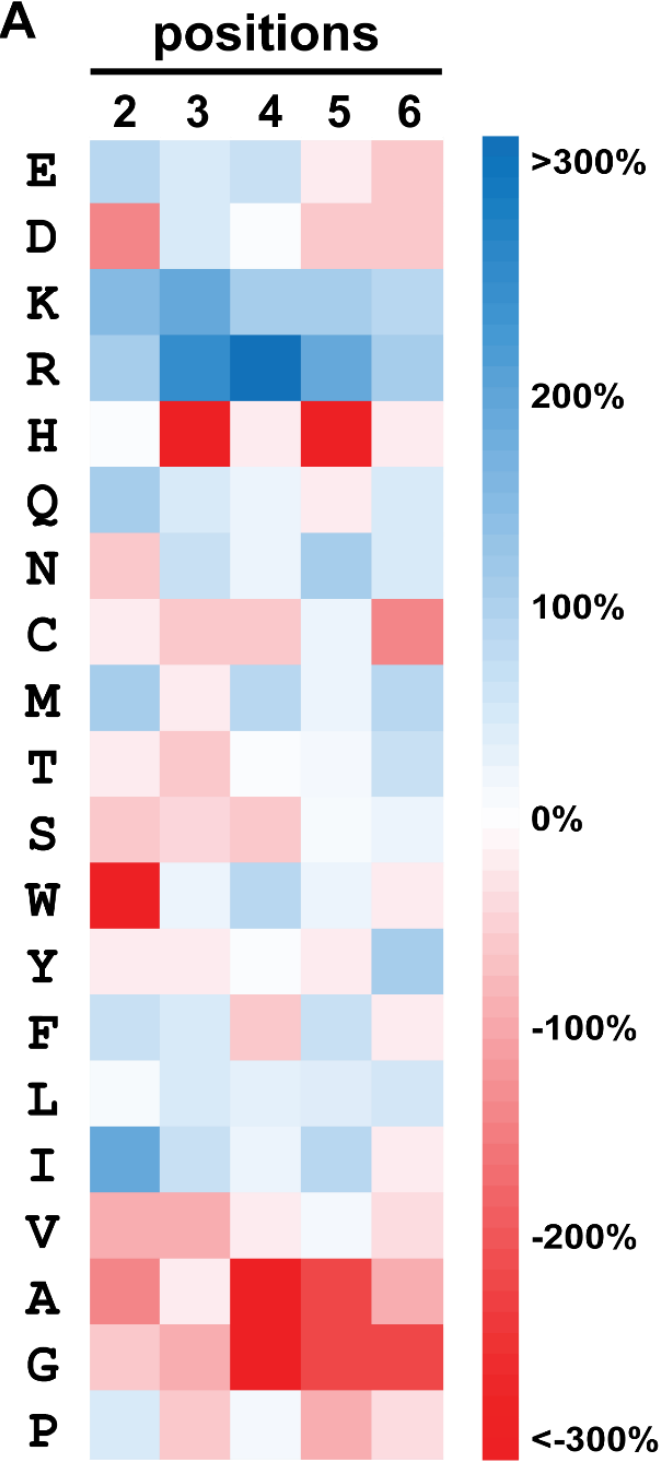

B
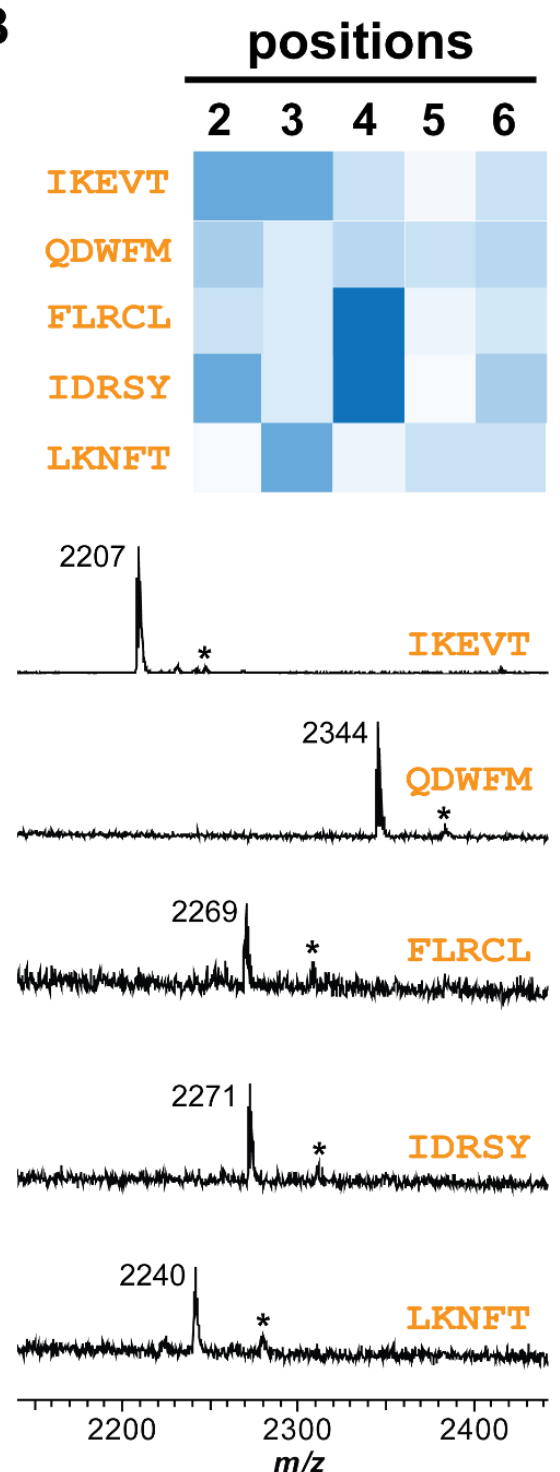

Figure S34. Heatmap analysis of FusC nonsubstrates. (A) The heatmap shows the percent difference between the observed occurrence of each residue on each position with expected occurrence based on the NNK codon. The analysis was conducted based on 154 sequence-confirmed nonsubstrates. The values are indicated on the color scheme. Blue-colored residues appeared more than expected in FusC nonsubstrates, while red-colored residues appeared less than expected. (B) Anticipated FusC nonsubstrates based on the heatmap. All five residues in designed sequences are predicted to be favored in FusC nonsubstrates. No signal was detected in CFB. The formation of their corresponding linear core peptides was detected by MALDI-TOF-MS from the PURExpress reaction, in which the precursor peptides were synthesized and reacted with purified FusB, C, and E. Orange depicts the varied sequence of positions 2-6 of the FusA core region. The mass label corresponds to the $[\mathrm{M}+\mathrm{H}]^{+}$ion of the uncyclized linear core peptide. ${ }^{*}$ indicates the $[\mathrm{M}+\mathrm{K}]^{+}$ion of the linear core peptide. 

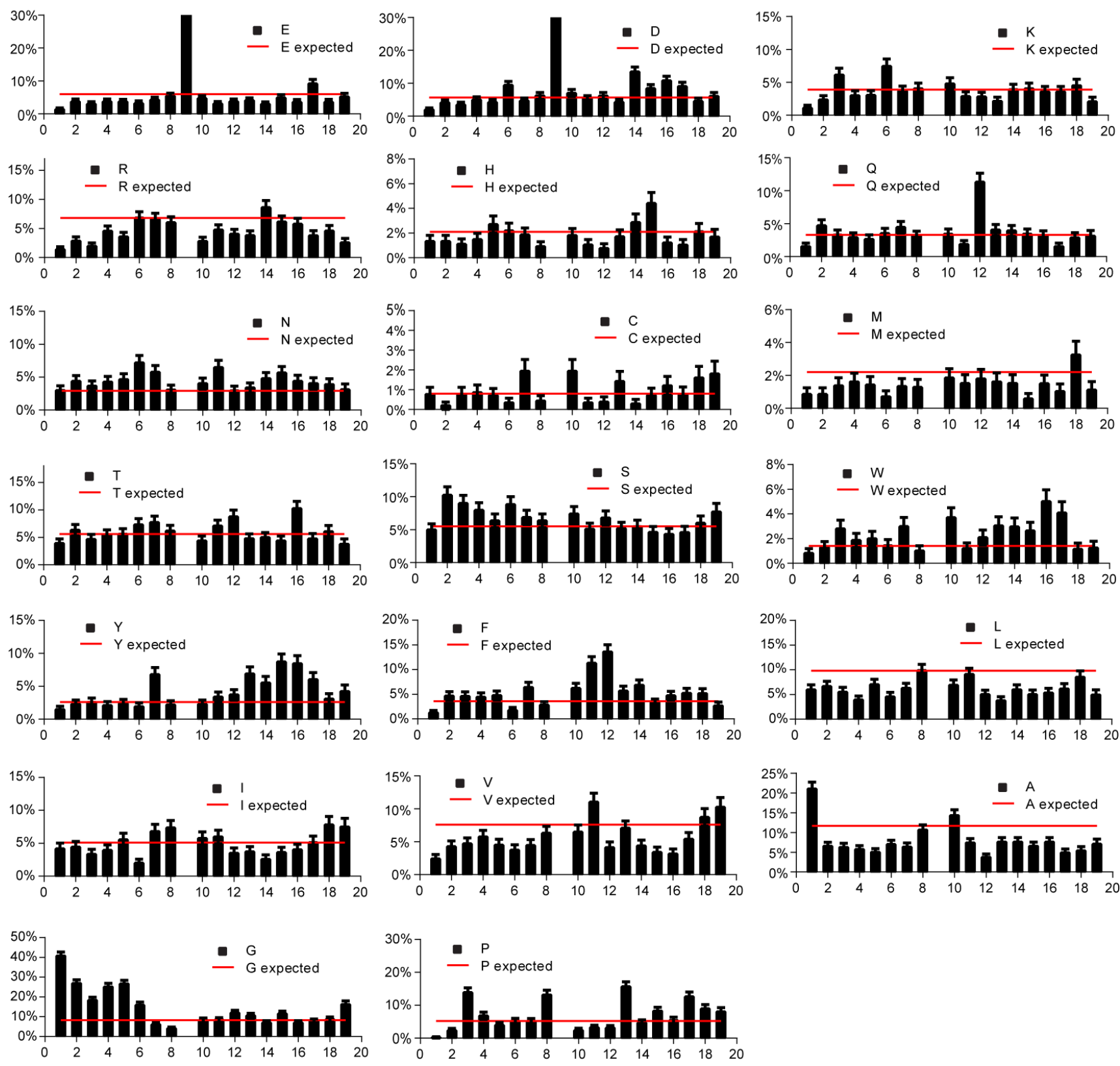

Figure S35. Position-specific amino acid analysis for lasso peptides. Shown are the observed frequencies (Y-axis) of the indicated amino acids within the set of unique lasso peptide core sequences (identical cores are only counted once). These values are compared to the expected frequency (red line), derived from the weighted average of the codon frequencies used by the most frequent genus that are predicted to encode lasso peptides (Table S7). Error bars represent the 95\% confidence interval. $\mathrm{X}$-axis values represent the position in the lasso peptide core region (positions 1 and 9 form the macrolactam). 


\section{References}

(1) Higuchi, R.; Krummel, B.; Saiki, R. A General Method of In vitro Preparation and Specific Mutagenesis of DNA Fragments: Study of Protein and DNA Interactions. Nucleic Acids Res. 1988, 16 (15), 7351-7367.

(2) DiCaprio, A. J.; Firouzbakht, A.; Hudson, G. A.; Mitchell, D. A. Enzymatic Reconstitution and Biosynthetic Investigation of the Lasso Peptide Fusilassin. J. Am. Chem. Soc. 2019, 141 (1), 290-297.

(3) Sun, Z. Z.; Hayes, C. A.; Shin, J.; Caschera, F.; Murray, R. M.; Noireaux, V. Protocols for Implementing an Escherichia coli Based TX-TL Cell-Free Expression System for Synthetic Biology. J. Vis. Exp. 2013, No. 79, 50762.

(4) Kunakom, S.; Eustáquio, A. S. Heterologous Production of Lasso Peptide Capistruin in a Burkholderia Host. ACS Synth. Biol. 2020, 9 (2), 241-248.

(5) Bradford, M. M. A Rapid and Sensitive Method for the Quantitation of Microgram Quantities of Protein Utilizing the Principle of Protein-Dye Binding. Anal. Biochem. 1976, 72 (1-2), 248254.

(6) Sun, Z. Z.; Yeung, E.; Hayes, C. A.; Noireaux, V.; Murray, R. M. Linear DNA for Rapid Prototyping of Synthetic Biological Circuits in an Escherichia coli Based TX-TL Cell-Free System. ACS Synth. Biol. 2014, 3 (6), 387-397.

(7) Fleiss, J. L.; Levin, B.; Paik, M. C. Statistical Methods for Rates and Proportions; Shewart, W. A., Wilks, S. S., Series Eds.; Wiley Series in Probability and Statistics; John Wiley \& Sons, Inc.: Hoboken, NJ, USA, 2003.

(8) Pettersen, E. F.; Goddard, T. D.; Huang, C. C.; Couch, G. S.; Greenblatt, D. M.; Meng, E. C.; Ferrin, T. E. UCSF Chimera--a Visualization System for Exploratory Research and Analysis. $J$. Comput. Chem. 2004, 25 (13), 1605-1612.

(9) Sanner, M. F.; Olson, A. J.; Spehner, J. C. Reduced Surface: An Efficient Way to Compute Molecular Surfaces. Biopolymers 1996, 38 (3), 305-320.

(10) Altschul, S. Gapped BLAST and PSI-BLAST: A New Generation of Protein Database Search Programs. Nucleic Acids Res. 1997, 25 (17), 3389-3402.

(11) Finn, R. D.; Coggill, P.; Eberhardt, R. Y.; Eddy, S. R.; Mistry, J.; Mitchell, A. L.; Potter, S. C.; Punta, M.; Qureshi, M.; Sangrador-Vegas, A.; Salazar, G. A.; Tate, J.; Bateman, A. The Pfam Protein Families Database: Towards a More Sustainable Future. Nucleic Acids Res. 2016, 44 (D1), D279-D285.

(12) Tietz, J. I.; Schwalen, C. J.; Patel, P. S.; Maxson, T.; Blair, P. M.; Tai, H.-C.; Zakai, U. I.; Mitchell, D. A. A New Genome-Mining Tool Redefines the Lasso Peptide Biosynthetic Landscape. Nat. Chem. Biol. 2017, 13 (5), 470-478. 
(13) Hegemann, J. D.; Zimmermann, M.; Zhu, S.; Steuber, H.; Harms, K.; Xie, X.; Marahiel, M. A. Xanthomonins I-III: A New Class of Lasso Peptides with a Seven-Residue Macrolactam Ring. Angew. Chem. Int. Ed Engl. 2014, 53 (8), 2230-2234.

(14) Shannon, C. E. A Mathematical Theory of Communication. Bell Syst. Tech. J. 1948, 27 (3), 379-423.

(15) Ashkenazy, H.; Abadi, S.; Martz, E.; Chay, O.; Mayrose, I.; Pupko, T.; Ben-Tal, N. ConSurf 2016: An Improved Methodology to Estimate and Visualize Evolutionary Conservation in Macromolecules. Nucleic Acids Res. 2016, 44 (W1), W344-350.

(16) Capra, J. A.; Singh, M. Predicting Functionally Important Residues from Sequence Conservation. Bioinformatics 2007, 23 (15), 1875-1882.

(17) Athey, J.; Alexaki, A.; Osipova, E.; Rostovtsev, A.; Santana-Quintero, L. V.; Katneni, U.; Simonyan, V.; Kimchi-Sarfaty, C. A New and Updated Resource for Codon Usage Tables. BMC Bioinformatics 2017, 18 (1), 391.

(18) Knappe, T. A.; Linne, U.; Zirah, S.; Rebuffat, S.; Xie, X.; Marahiel, M. A. Isolation and Structural Characterization of Capistruin, a Lasso Peptide Predicted from the Genome Sequence of Burkholderia thailandensis E264. J. Am. Chem. Soc. 2008, 130 (34), 11446-11454.

(19) Crooks, G. E. WebLogo: A Sequence Logo Generator. Genome Res. 2004, 14 (6), 1188-1190. 